\title{
REGULARITY OF WEAKLY WELL POSED HYPERBOLIC MIXED PROBLEMS WITH CHARACTERISTIC BOUNDARY
}

\author{
ALESSANDRO MORANDO \\ Dipartimento di Matematica-Facoltà di Ingegneria \\ Via Valotti 9, 25133 Brescia, Italy \\ alessandro.morando@ing.unibs.it \\ PAOLO SECCHI \\ Dipartimento di Matematica-Facoltà di Ingegneria \\ Via Valotti 9, 25133 Brescia, Italy \\ paolo.secchi@ing.unibs.it \\ Received (Day Mth. Year) \\ Revised (Day Mth. Year) \\ Communicated by [editor]
}

\begin{abstract}
We study the mixed initial-boundary value problem for a linear hyperbolic system with characteristic boundary of constant multiplicity. We assume the problem to be "weakly" well posed, in the sense that a unique $L^{2}$-solution exists, for sufficiently smooth data, and obeys an a priori energy estimate with a finite loss of conormal regularity. This is the case of problems that do not satisfy the uniform Kreiss-Lopatinskir condition in the hyperbolic region of the frequency domain. Under the assumption of the loss of one conormal derivative we obtain the regularity of solutions, in the natural framework of weighted anisotropic Sobolev spaces, provided the data are sufficiently smooth.
\end{abstract}

Keywords: Symmetrizable systems, symmetric hyperbolic systems, mixed initialboundary value problem, weak well posedness, loss of derivatives, characteristic boundary, anisotropic Sobolev spaces, tangential regularity.

Mathematics Subject Classification 2010: 35L40, 35L50.

\section{Introduction and main results}

For $n \geq 2$, let $\mathbb{R}_{+}^{n}$ denote the $n$-dimensional positive half-space

$$
\mathbb{R}_{+}^{n}:=\left\{x=\left(x_{1}, x^{\prime}\right), x_{1}>0, x^{\prime}:=\left(x_{2}, \ldots, x_{n}\right) \in \mathbb{R}^{n-1}\right\} .
$$

The boundary of $\mathbb{R}_{+}^{n}$ will be sistematically identified with $\mathbb{R}_{x^{\prime}}^{n-1}$. For $T>0$ we set $\left.Q_{T}=\mathbb{R}_{+}^{n} \times\right] 0, T\left[\right.$ and $\left.\Sigma_{T}=\mathbb{R}^{n-1} \times\right] 0, T\left[\right.$; we also set $\left.\Omega_{T}=\mathbb{R}_{+}^{n} \times\right]-\infty, T[$ and $\left.\omega_{T}=\mathbb{R}^{n-1} \times\right]-\infty, T\left[\right.$. If time $t$ spans the whole real line $\mathbb{R}$, we set $Q=\mathbb{R}_{+}^{n} \times \mathbb{R}_{t}$ and $\Sigma=\mathbb{R}^{n-1} \times \mathbb{R}_{t}$. We are interested in the following initial-boundary value problem 
2 A. Morando \&3 P. Secchi

(shortly written IBVP)

$$
\begin{aligned}
L u=F & \text { in } Q_{T}, \\
M u=G & \text { on } \Sigma_{T}, \\
u_{\mid t=0}=f & \text { in } \mathbb{R}_{+}^{n},
\end{aligned}
$$

where $L$ is a first order linear partial differential operator

$$
L=\partial_{t}+\sum_{i=1}^{n} A_{i}(x, t) \partial_{i}+B(x, t)
$$

$\partial_{t}:=\frac{\partial}{\partial t}$ and $\partial_{i}:=\frac{\partial}{\partial x_{i}}, i=1, \ldots, n$.

The coefficients $A_{i}, B$, for $i=1, \ldots, n$, are real $N \times N$ matrix-valued functions, defined on $Q$. The unknown $u=u(x, t)$, and the data $F=F(x, t), G=G(x, t)$, $f=f(x)$ are vector-valued functions with $N$ components. $M$ is a given real $d \times N$ matrix-valued function; $M$ is supposed to have maximal constant rank $d$.

We study the problem (1.1)-(1.3) under the following assumptions. The function spaces involved in (4), (5) and in the statement of Theorems 1.1, 1.2 below, as well as the norms appearing in (1.9)-(1.11), (1.13), will be described in the next Section 2. The square brackets [ ] of a real number denote its integer part.

(1) $L$ is Friedrichs symmetrizable, namely there exists a matrix $S_{0}$, definite positive on $\bar{Q}$ (there exists a constant $\rho>0$ such that $S_{0}(x, t) \geq \rho$ for every $(x, t) \in$ $Q)$, symmetric and such that the matrices $S_{0} A_{i}$, for $i=1, \ldots, n$, are also symmetric.

(2) The IBVP is characteristic of constant multiplicity $1 \leq r<N$. We assume that the coefficient $A_{1}$ of the normal derivative in $L$ displays the block-wise structure

$$
A_{1}(x)=\left(\begin{array}{cc}
A_{1}^{I, I} & A_{1}^{I, I I} \\
A_{1}^{I I, I} & A_{1}^{I I, I I}
\end{array}\right),
$$

where $A_{1}^{I, I}, A_{1}^{I, I I}, A_{1}^{I I, I}, A_{1}^{I I, I I}$ are respectively $r \times r, r \times(N-r),(N-r) \times r$, $(N-r) \times(N-r)$ sub-matrices, such that

$$
A_{1 \mid x_{1}=0}^{I, I I}=0, \quad A_{1 \mid x_{1}=0}^{I I, I}=0, \quad A_{1 \mid x_{1}=0}^{I I, I I}=0,
$$

and $A_{1}^{I, I}$ is uniformly invertible on the boundary $\Sigma$, namely there exists a real positive constant $\mu$ such that $\left|\operatorname{det} A_{1}^{I, I}(x, t)\right| \geq \mu$, for any $(x, t) \in \Sigma$. According to the representation above, we split the unknown $u$ as $u=\left(u^{I}, u^{I I}\right) ; u^{I} \in \mathbb{R}^{r}$ and $u^{I I} \in \mathbb{R}^{N-r}$ are said respectively the noncharacteristic and the characteristic components of $u$.

(3) The matrix $M$ has the form $M=\left(I_{d} 0\right)$, where $I_{d}$ denotes the identity matrix of order $d, 0$ is the zero matrix of size $d \times(N-d)$, and $d \leq r$ is the (constant) number of positive eigenvalues of $A_{1 \mid\left\{x_{1}=0\right\}}^{I, I}$ (the so-called incoming characteristics of problem (1.1)-(1.3)). 
(4) Existence of the $L^{2}$ weak solution. Assume that $S_{0}, A_{i} \in W^{2, \infty}(Q)$, for $i=1, \ldots, n$. For all $T>0$ and matrices $B \in W^{1, \infty}\left(\Omega_{T}\right)$, there exist constants $\gamma_{0} \geq 1$ and $C_{0}>0$ (that depend on $\left.T, \rho, \mu,\left\|S_{0}\right\|_{W^{2, \infty}\left(\Omega_{T}\right)},\left\|A_{j}\right\|_{W^{2, \infty}\left(\Omega_{T}\right)},\|B\|_{W^{1, \infty}\left(\Omega_{T}\right)}\right)$ such that for all $\gamma \geq \gamma_{0}$ and $F \in H_{\text {tan, },}^{1}\left(\Omega_{T}\right), G \in H_{\gamma}^{1}\left(\omega_{T}\right)$, vanishing for $t<0$, the boundary value problem (shortly written BVP)

$$
\begin{aligned}
L u & =F & & \text { in } \Omega_{T}, \\
M u & =G & & \text { on } \omega_{T},
\end{aligned}
$$

with $B$ in $L$, admits a unique solution $u \in L^{2}\left(\Omega_{T}\right)$, vanishing for $t<0$, such that $u_{\mid \omega_{T}}^{I} \in L^{2}\left(\omega_{T}\right)$. Furthermore $u \in C\left([0, T] ; L^{2}\left(\mathbb{R}_{+}^{n}\right)\right)$, and it satisfies an a priori estimate of the form

$$
\begin{aligned}
& \gamma\left\|u_{\gamma}\right\|_{L^{2}\left(\Omega_{t}\right)}^{2}+\left\|u_{\gamma}(t)\right\|_{L^{2}\left(\mathbb{R}_{+}^{n}\right)}^{2}+\left\|u_{\gamma \mid \omega_{t}}^{I}\right\|_{L^{2}\left(\omega_{t}\right)}^{2} \\
& \leq C_{0}\left(\frac{1}{\gamma^{3}}\left\|F_{\gamma}\right\|_{H_{t a n, \gamma}^{1}\left(\Omega_{t}\right)}^{2}+\frac{1}{\gamma^{2}}\left\|G_{\gamma}\right\|_{H_{\gamma}^{1}\left(\omega_{t}\right)}^{2}\right)
\end{aligned}
$$

for all $\gamma \geq \gamma_{0}$ and $0<t \leq T$, where we have set $u_{\gamma}=e^{-\gamma t} u, F_{\gamma}=e^{-\gamma t} F, G_{\gamma}=$ $e^{-\gamma t} G$.

Furthermore, if $T=+\infty$, for all matrices $B_{1} \in W^{1, \infty}(Q)$ and all conormal pseudo-differential operators $B_{2}$ with symbol of degree 0 (in $\Gamma^{0}$ ), there exist constants $\gamma_{0}^{\prime} \geq 1$ and $C_{0}^{\prime}>0$ (that depend on $\rho, \mu,\left\|S_{0}\right\|_{W^{2, \infty}(Q)},\left\|A_{j}\right\|_{W^{2, \infty}(Q)},\left\|B_{1}\right\|_{W^{1, \infty}(Q)}$, and on a finite number of seminorms of the symbol of $B_{2}$ ) such that for all $\gamma \geq \gamma_{0}^{\prime}$ and for all $F \in$ $e^{\gamma t} H_{\text {tan, } \gamma}^{1}(Q), G \in e^{\gamma t} H_{\gamma}^{1}(\Sigma)$, the BVP (1.7), (1.8) on $Q$, with $B=B_{1}+B_{2}$ in $L$, admits a unique solution $u \in e^{\gamma t} L^{2}(Q)$ such that $u_{\mid \Sigma}^{I} \in e^{\gamma t} L^{2}(\Sigma)$. Furthermore $u$ satisfies the a priori estimate

$$
\gamma\left\|u_{\gamma}\right\|_{L^{2}(Q)}^{2}+\left\|u_{\gamma \mid \Sigma}^{I}\right\|_{L^{2}(\Sigma)}^{2} \leq C_{0}^{\prime}\left(\frac{1}{\gamma^{3}}\left\|F_{\gamma}\right\|_{H_{t a n, \gamma}^{1}(Q)}^{2}+\frac{1}{\gamma^{2}}\left\|G_{\gamma}\right\|_{H_{\gamma}^{1}(\Sigma)}^{2}\right) .
$$

(5) Given $T>0$, and matrices $\left(S_{0}, A_{i}\right) \in \mathcal{C}_{T}\left(H_{*, \gamma}^{\sigma}\right) \times \mathcal{C}_{T}\left(H_{*, \gamma}^{\sigma}\right)$, where $\sigma \geq[(n+$ 1) $/ 2]+4$, and enjoying properties (1) - (4) on $Q$, let $\left(S_{0}^{(k)}, A_{i}^{(k)}\right)$ be $C^{\infty}$ matrixvalued functions converging, as $k \rightarrow \infty$, to $\left(S_{0}, A_{i}\right)$ in $\mathcal{C}_{T}\left(H_{*, \gamma}^{\sigma}\right) \times \mathcal{C}_{T}\left(H_{*, \gamma}^{\sigma}\right)$ on $[0, T]$, and in $W^{2, \infty}(Q) \times W^{2, \infty}(Q)$ on $\mathbb{R}_{t}$. Assume also that $\left(S_{0}^{(k)}, A_{i}^{(k)}\right)$ satisfy properties (1), (2) on $Q$. Then, for $k$ sufficiently large, property (4) holds also for the approximating problems with coefficients $\left(S_{0}^{(k)}, A_{i}^{(k)}\right)$.

When an IBVP admits the solution $u$ enjoying an a priori estimate of type (1.9) or (1.10), with $F=L u, G=M u$, the IBVP is weakly $L^{2}$-well posed. This is the case of problems that do not satisfy the uniform Kreiss-Lopatinskir condition. More specifically, the loss of derivatives as in (1.9), (1.10) occurs when the Kreiss-Lopatinskil determinant has one simple root in the hyperbolic region of the frequency domain, see e.g. [3,4] for the definitions. In [10], Coulombel and Guès 
show that the loss of regularity in (1.9), (1.10) in such a case is optimal. They also prove that the well posedness result with loss of regularity is independent of Lipschitzean zero order terms $B$ but is not independent of bounded zero order terms. This is a major difference with the strongly well posed case where there is no loss of derivatives (and one can treat lower order terms as source terms in energy estimates). Thus the stability of the problem under lower order perturbations is no longer a trivial consequence of the well posedness itself, and we assume it as an additional hypothesis about the IBVP, see (4). Under an a priori estimate of this form, Coulombel [9] has proven the well posedness of the problem, namely the existence of the $L^{2}$ solution for all $H^{1}$ data.

As for (5), hyperbolic IBVP that do not satisfy the uniform Kreiss-Lopatinski1 condition in the hyperbolic region as above belong to the WR class defined by Benzoni-Gavage, Rousset, Serre and Zumbrun [3]. This class of problems is stable with respect to small perturbations of the coefficients $A_{j}, B$, in agreement with (5). Examples of problems where the uniform Kreiss-Lopatinskiu condition breaks down are provided by elastodynamics (with the well-known Rayleigh waves $[23,28]$ ), shock waves or contact discontinuities in compressible fluid mechanics, see e.g. [15,12]. An a priori estimate similar to (1.9), (1.10) holds for linearized compressible vortex sheets, see Coulombel and Secchi $[11,12,13]$, provided that $S_{0}, A_{i} \in W^{2, \infty}(Q)$ and $B \in W^{1, \infty}(Q)$.

Under the assumptions (1)-(4) it is not hard to obtain the $L^{2}$ solvability of the nonhomogeneous IBVP (1.1)-(1.3) on $[0, T]$, with initial data $f \neq 0$, that we state in the following theorem.

Theorem 1.1. Assume that problem (1.1)-(1.3) obeys the assumptions (1)-(4). For all $T>0$ and matrices $B \in W^{1, \infty}\left(Q_{T}\right)$, there exist constants (denoted as above) $\gamma_{0} \geq 1$ and $C_{0}>0$ (that depend on $T, \rho, \mu,\left\|S_{0}\right\|_{W^{2, \infty}\left(Q_{T}\right)},\left\|A_{j}\right\|_{W^{2, \infty}\left(Q_{T}\right)}$ and $\left.\|B\|_{W^{1, \infty}\left(Q_{T}\right)}\right)$ such that for all $F \in H_{\text {tan, } \gamma}^{1}\left(Q_{T}\right), G \in H_{\gamma}^{1}\left(\Sigma_{T}\right), f \in H_{\text {tan, } \gamma}^{1}\left(\mathbb{R}_{+}^{n}\right)$ with $f^{(1)}:=F_{\mid t=0}-\sum_{i=1}^{n} A_{i \mid t=0} \partial_{i} f-B_{\mid t=0} f \in L^{2}\left(\mathbb{R}_{+}^{n}\right)$, and such that $M f=G_{\mid t=0}$ on $\mathbb{R}^{n-1}$, the problem (1.1)-(1.3), with data $(F, G, f)$, admits a unique solution $u \in L^{2}\left(Q_{T}\right)$ such that $u_{\mid \Sigma_{T}}^{I} \in L^{2}\left(\Sigma_{T}\right)$. Furthermore $u \in C\left([0, T] ; L^{2}\left(\mathbb{R}_{+}^{n}\right)\right)$, and it satisfies an a priori estimate of the form

$$
\begin{aligned}
& \gamma\left\|u_{\gamma}\right\|_{L^{2}\left(Q_{t}\right)}^{2}+\left\|u_{\gamma}(t)\right\|_{L^{2}\left(\mathbb{R}_{+}^{n}\right)}^{2}+\left\|u_{\gamma \mid \Sigma_{t}}^{I}\right\|_{L^{2}\left(\Sigma_{t}\right)}^{2} \\
& \leq C_{0}\left(\frac{1}{\gamma^{3}}\left\|F_{\gamma}\right\|_{H_{t a n, \gamma}^{1}\left(Q_{t}\right)}^{2}+\frac{1}{\gamma^{2}} \mid\|f\|_{1, t a n, \gamma}^{2}+\frac{1}{\gamma^{2}}\left\|G_{\gamma}\right\|_{H_{\gamma}^{1}\left(\Sigma_{t}\right)}^{2}\right)
\end{aligned}
$$

for all $\gamma \geq \gamma_{0}$ and $0<t \leq T$.

The proof of Theorem 1.1 will be given in Appendix Appendix A.

In order to study the regularity of solutions to the IBVP (1.1)-(1.3), we need to impose some compatibility conditions on the data $F, G, f$. The compatibility conditions are defined in the usual way, see [22]. Given the equation (1.1), we recursively define $f^{(h)}$ by formally taking $h-1$ time derivatives of $L u=F$, solving for $\partial_{t}^{h} u$ and evaluating it at $t=0$. For $h=0$ we set $f^{(0)}:=f$. The compatibility condition 
of order $m \geq 0$ for the IBVP (1.1)-(1.3) reads as

$$
M f^{(h)}=\partial_{t}^{h} G_{\mid t=0}, \quad \text { on } \mathbb{R}^{n-1}, h=0, \ldots, m .
$$

The aim of this paper is to prove the following regularity theorem.

Theorem 1.2. Let $m \in \mathbb{N}, m \geq 1$, and $s=\max \{m+1,[(n+1) / 2]+7\}$. Given $T>0$, assume that $S_{0}, A_{j} \in \mathcal{C}_{T}\left(H_{*, \gamma}^{s}\right)$, for $j=1, \ldots, n$, and $B \in \mathcal{C}_{T}\left(H_{*, \gamma}^{s-1}\right)$ (or $B \in \mathcal{C}_{T}\left(H_{*, \gamma}^{s}\right)$ if $\left.m=s\right)$. Assume also that the assumptions (1)-(5) are satisfied. Then for all $F \in H_{*, \gamma}^{m+1}\left(Q_{T}\right)$, with $\partial_{t}^{i} F_{\mid t=0} \in H_{\gamma}^{m-i}\left(\mathbb{R}_{+}^{n}\right)$ for $i=0, \ldots, m-1, G \in$ $H_{\gamma}^{m+1}\left(\Sigma_{T}\right), f \in H_{\gamma}^{m+1}\left(\mathbb{R}_{+}^{n}\right)$, satisfying the compatibility condition (1.12) of order $m$, the unique solution $u$ to $(1.1)-(1.3)$, with data $(F, G, f)$, belongs to $\mathcal{C}_{T}\left(H_{*, \gamma}^{m}\right)$ and $u_{\mid \Sigma_{T}}^{I} \in H_{\gamma}^{m}\left(\Sigma_{T}\right)$. Moreover $u$ satisfies the a priori estimate

$$
\begin{aligned}
& \gamma\left\|u_{\gamma}\right\|_{H_{*, \gamma}^{m}\left(Q_{T}\right)}^{2}+\left.\max _{t \in[0, T]}\left\|u_{\gamma}(t)\right\|\right|_{m, *, \gamma} ^{2}+\left\|u_{\gamma \mid \Sigma_{T}}^{I}\right\|_{H_{\gamma}^{m}\left(\Sigma_{T}\right)}^{2} \\
& \leq C_{m}\left(\frac{1}{\gamma^{2}}\|\| f\left\|_{m+1, *, \gamma}^{2}+\frac{1}{\gamma^{3}}\right\| F_{\gamma}\left\|_{H_{*, \gamma}^{m+1}\left(Q_{T}\right)}^{2}+\frac{1}{\gamma^{2}}\right\| G_{\gamma} \|_{H_{\gamma}^{m+1}\left(\Sigma_{T}\right)}^{2}\right)
\end{aligned}
$$

with a constant $C_{m}>0$ depending only on $A_{j}, B$.

The function spaces involved in the statement above, as well as the norms appearing in (1.13), will be described in the next Section 2.

In [18], the regularity of weak solutions to the characteristic IBVP (1.1)-(1.3) is studied, under the assumption that the problem is strongly $L^{2}$-well posed, namely that a unique $L^{2}$-solution exists for arbitrarily given $L^{2}$-data, and the solution obeys an a priori energy inequality without loss of regularity with respect to the data; this means that the $L^{2}$-norms of the interior and boundary values of the solution are measured by the $L^{2}$-norms of the corresponding data $F, G, f$. The statement of Theorem 1.2 extends the result of [18] to the case where only a weak well posedness property is satisfied by the IBVP (1.1)-(1.3). Here, the $L^{2}$-solvability of (1.1)-(1.3) requires an additional regularity of the data $F, G, f$, cfr. (4). Correspondingly, the regularity of the solution of order $m$ is achieved provided the data have a regularity of order $m+1$.

To prove the result of [18], the solution $u$ to (1.1)-(1.3) is regularized by a family of tangential mollifiers $J_{\varepsilon}, 0<\varepsilon<1$, defined by Nishitani and Takayama in [19] as a suitable combination of the operator $\sharp$ (see Section 3) and the standard Friedrichs'mollifiers. The essential point of the analysis performed in [18] is to notice that the mollified solution $J_{\varepsilon} u$ solves the same problem (1.1)-(1.3), as the original solution $u$. The data of the problem for $J_{\varepsilon} u$ come from the regularization, by $J_{\varepsilon}$, of the data involved in the original problem for $u$; furthermore, an additional term $\left[J_{\varepsilon}, L\right] u$, where $\left[J_{\varepsilon}, L\right]$ is the commutator between the differential operator $L$ and the tangential mollifier $J_{\varepsilon}$, appears into the equation satisfied by $J_{\varepsilon} u$. Because the strong $L^{2}$-well posedness is preserved under lower order perturbations, actually this term can be incorporated into the source term of the equation satisfied by $J_{\varepsilon} u$.

In the case of Theorem 1.2, where the $L^{2}$ a priori estimate exhibits a finite loss of regularity with respect to the data, this technique seems to be unsuccesful, since 
$\left[J_{\varepsilon}, L\right] u$ cannot be absorbed into the right-hand side without losing derivatives on the solution $u$; on the other hand it seems that the same term cannot be merely reduced to a lower order term involving the smoothed solution $J_{\varepsilon} u$, as well.

These observations lead to develop another technique, where the tangential mollifier $J_{\varepsilon}$ is replaced by the family of operators (3.26), involved in the characterization of regularity given by Proposition 3.2. Instead of studying the problem satisfied by the smoothed solution $J_{\varepsilon} u$, here we consider the problem satisfied by $\lambda_{\delta}^{m-1, \gamma}(Z) u$. As before, a new term $\left[\lambda_{\delta}^{m-1, \gamma}(Z), L\right] u$ appears which takes account of the commutator between the differential operator $L$ and the conormal operator $\lambda_{\delta}^{m-1, \gamma}(Z)$. Since we assume the weak well posedness of the IBVP (1.1)-(1.3) to be preserved under lower order terms, the approach is to treat the commutator $\left[\lambda_{\delta}^{m-1, \gamma}(Z), L\right] u$ as a lower order term within the interior equation for $\lambda_{\delta}^{m-1, \gamma}(Z) u$ (see (4.16)); this is made possible by taking advantage from the invertibility of the operator $\lambda_{\delta}^{m-1, \gamma}(Z)$.

The paper is organized as follows. In Section 2 we introduce the function spaces and some notations. In Section 3 we give some technical results useful for the proof of the tangential regularity, discussed in Section 4 . Sections 5 and 6 contain the proof of the normal regularity for $m=1$ and $m \geq 2$, respectively. The proof of Theorem 1.1 is given in Appendix Appendix A. Some useful properties of the $\gamma$-dependent spaces $H_{*, \gamma}^{m}\left(\mathbb{R}_{+}^{n}\right)$ are proved in Appendix Appendix B.

\section{Function Spaces}

The purpose of this Section is to introduce the main function spaces to be used in the following and collect their basic properties.

\subsection{Weighted Sobolev spaces}

For $\gamma \geq 1$ and $s \in \mathbb{R}$, we set

$$
\lambda^{s, \gamma}(\xi):=\left(\gamma^{2}+|\xi|^{2}\right)^{s / 2}
$$

and, in particular, $\lambda^{s, 1}:=\lambda^{s}$.

Throughout the paper, for real $\gamma \geq 1, H_{\gamma}^{s}\left(\mathbb{R}^{n}\right)$ will denote the Sobolev space of order $s$, equipped with the $\gamma$-depending norm $\|\cdot\|_{s, \gamma}$ defined by

$$
\|u\|_{s, \gamma}^{2}:=(2 \pi)^{-n} \int_{\mathbb{R}^{n}} \lambda^{2 s, \gamma}(\xi)|\widehat{u}(\xi)|^{2} d \xi,
$$

$\widehat{u}$ being the Fourier transform of $u$. The norms defined by (2.2), with different values of the parameter $\gamma$, are equivalent each other. For $\gamma=1$ we set for brevity $\|\cdot\|_{s}:=\|\cdot\|_{s, 1}$ (and, accordingly, the standard Sobolev space $H^{s}\left(\mathbb{R}^{n}\right):=H_{1}^{s}\left(\mathbb{R}^{n}\right)$ ). For $s \in \mathbb{N}$, the norm in (2.2) turns to be equivalent, uniformly with respect to $\gamma$, to the norm $\|\cdot\|_{H_{\gamma}^{s}\left(\mathbb{R}^{n}\right)}$ defined by

$$
\|u\|_{H_{\gamma}^{s}\left(\mathbb{R}^{n}\right)}^{2}:=\sum_{|\alpha| \leq s} \gamma^{2(s-|\alpha|)}\left\|\partial^{\alpha} u\right\|_{L^{2}\left(\mathbb{R}^{n}\right)}^{2} .
$$


An useful remark is that

$$
\|u\|_{s, \gamma} \leq \gamma^{s-r}\|u\|_{r, \gamma},
$$

for arbitrary $s \leq r$ and $\gamma \geq 1$.

\subsection{Conormal Sobolev spaces}

Let us introduce some classes of function spaces of Sobolev type, defined over the half-space $\mathbb{R}_{+}^{n}$. For $j=1,2, \ldots, n$, we set

$$
Z_{1}:=x_{1} \partial_{1}, \quad Z_{j}:=\partial_{j}, \text { for } j \geq 2 .
$$

Then, for every multi-index $\alpha=\left(\alpha_{1}, \ldots, \alpha_{n}\right) \in \mathbb{N}^{n}$, the conormal derivative $Z^{\alpha}$ is defined by

$$
Z^{\alpha}:=Z_{1}^{\alpha_{1}} \ldots Z_{n}^{\alpha_{n}}
$$

we also write $\partial^{\alpha}=\partial_{1}^{\alpha_{1}} \ldots \partial_{n}^{\alpha_{n}}$ for the usual partial derivative corresponding to $\alpha$. Given an integer $m \geq 1$ the conormal Sobolev space $H_{t a n}^{m}\left(\mathbb{R}_{+}^{n}\right)$ is defined as the set of functions $u \in L^{2}\left(\mathbb{R}_{+}^{n}\right)$ such that $Z^{\alpha} u \in L^{2}\left(\mathbb{R}_{+}^{n}\right)$, for all multi-indices $\alpha$ with $|\alpha| \leq m$. Agreeing with the notations set for the usual Sobolev spaces, for $\gamma \geq 1, H_{t a n, \gamma}^{m}\left(\mathbb{R}_{+}^{n}\right)$ will denote the conormal space of order $m$ equipped with the $\gamma$-depending norm

$$
\|u\|_{H_{t a n, \gamma}^{m}\left(\mathbb{R}_{+}^{n}\right)}^{2}:=\sum_{|\alpha| \leq m} \gamma^{2(m-|\alpha|)}\left\|Z^{\alpha} u\right\|_{L^{2}\left(\mathbb{R}_{+}^{n}\right)}^{2}
$$

and we have $H_{t a n}^{m}\left(\mathbb{R}_{+}^{n}\right):=H_{t a n, 1}^{m}\left(\mathbb{R}_{+}^{n}\right)$.

\subsection{Anisotropic Sobolev spaces}

Keeping the same notations used above, for every positive integer $m$ the anisotropic Sobolev space $H_{*}^{m}\left(\mathbb{R}_{+}^{n}\right)$ is defined as

$$
H_{*}^{m}\left(\mathbb{R}_{+}^{n}\right):=\left\{w \in L^{2}\left(\mathbb{R}_{+}^{n}\right): \quad Z^{\alpha} \partial_{1}^{k} w \in L^{2}\left(\mathbb{R}_{+}^{n}\right),|\alpha|+2 k \leq m\right\} .
$$

For the sake of convenience we also set $H_{*}^{0}\left(\mathbb{R}_{+}^{n}\right)=H_{\text {tan }}^{0}\left(\mathbb{R}_{+}^{n}\right)=L^{2}\left(\mathbb{R}_{+}^{n}\right)$. For an extensive study of the anisotropic spaces $H_{*}^{m}\left(\mathbb{R}_{+}^{n}\right)$ we refer the reader to $[18,27]$ and references therein. We observe that

$$
\begin{aligned}
& H^{m}\left(\mathbb{R}_{+}^{n}\right) \hookrightarrow H_{*}^{m}\left(\mathbb{R}_{+}^{n}\right) \hookrightarrow H_{\text {tan }}^{m}\left(\mathbb{R}_{+}^{n}\right) \subset H_{\text {loc }}^{m}\left(\mathbb{R}_{+}^{n}\right), \\
& H_{*}^{m}\left(\mathbb{R}_{+}^{n}\right) \hookrightarrow H^{[m / 2]}\left(\mathbb{R}_{+}^{n}\right), \quad H_{*}^{1}\left(\mathbb{R}_{+}^{n}\right)=H_{\text {tan }}^{1}\left(\mathbb{R}_{+}^{n}\right)
\end{aligned}
$$

(except for $H_{l o c}^{m}\left(\mathbb{R}_{+}^{n}\right)$ all imbeddings are continuous). The anisotropic space $H_{*, \gamma}^{m}\left(\mathbb{R}_{+}^{n}\right)$ is the same space equipped with the $\gamma$-depending norm

$$
\|w\|_{H_{*, \gamma}^{m}\left(\mathbb{R}_{+}^{n}\right)}^{2}:=\sum_{|\alpha|+2 k \leq m} \gamma^{2(m-|\alpha|-2 k)}\left\|Z^{\alpha} \partial_{1}^{k} w\right\|_{L^{2}\left(\mathbb{R}_{+}^{n}\right)}^{2} .
$$

We have $H_{*}^{m}\left(\mathbb{R}_{+}^{n}\right)=H_{*, 1}^{m}\left(\mathbb{R}_{+}^{n}\right)$. The spaces $H_{\text {tan, } \gamma}^{m}\left(\mathbb{R}_{+}^{n}\right), H_{*, \gamma}^{m}\left(\mathbb{R}_{+}^{n}\right)$, endowed with their norms $(2.5),(2.7)$ respectively, are Hilbert spaces. 
In a similar way we define the anisotropic spaces $H_{t a n, \gamma}^{m}\left(Q_{T}\right), H_{*, \gamma}^{m}\left(Q_{T}\right)$, equipped with their natural norms.

Given any Banach space $X$, let $C^{j}([0, T] ; X)$ denote the space of all $X$-valued $j$-times continuously differentiable functions of $t$, for $t \in[0, T]$. We denote by $W^{j, \infty}(0, T ; X)$ the space of essentially bounded functions, together with the derivatives up to order $j$ on $[0, T]$, with values in $X$. We define the spaces

$\mathcal{C}_{T}\left(H_{*, \gamma}^{m}\right):=\bigcap_{j=0}^{m} C^{j}\left([0, T] ; H_{*, \gamma}^{m-j}\left(\mathbb{R}_{+}^{n}\right)\right), \quad \mathcal{C}_{T}\left(H_{\text {tan }, \gamma}^{m}\right):=\bigcap_{j=0}^{m} C^{j}\left([0, T] ; H_{\text {tan }, \gamma}^{m-j}\left(\mathbb{R}_{+}^{n}\right)\right)$,

with norms

$$
\|u\|_{\mathcal{C}_{T}\left(H_{*, \gamma}^{m}\right)}^{2}:=\sup _{t \in[0, T]}\left|\left\|u ( t ) \left|\| _ { m , * , \gamma } ^ { 2 } , \quad \| u \left\|_{\mathcal{C}_{T}\left(H_{t a n, \gamma}^{m}\right)}^{2}:=\sup _{t \in[0, T]}\left|\|u(t) \mid\|_{m, \tan , \gamma}^{2},\right.\right.\right.\right.\right.
$$

where

$\left.\||| u(t)\|\right|_{m, *, \gamma} ^{2}=\sum_{j=0}^{m}\left\|\partial_{t}^{j} u(t)\right\|_{H_{*, \gamma}^{m-j}\left(\mathbb{R}_{+}^{n}\right)}^{2}, \quad\|\| u(t)\left\|\left.\right|_{m, t a n, \gamma} ^{2}=\sum_{j=0}^{m}\right\| \partial_{t}^{j} u(t) \|_{H_{t a n, \gamma}^{m-j}\left(\mathbb{R}_{+}^{n}\right)}^{2}$.

For the initial data we set

$$
\||| f \mid\|_{m, *, \gamma}^{2}:=\sum_{j=0}^{m}\left\|f^{(j)}\right\|_{H_{*, \gamma}^{m-j}\left(\mathbb{R}_{+}^{n}\right)}^{2}, \quad\|\| f\left\|_{m, t a n, \gamma}^{2}:=\sum_{j=0}^{m}\right\| f^{(j)} \|_{H_{t a n, \gamma}^{m-j}\left(\mathbb{R}_{+}^{n}\right)}^{2} .
$$

Some useful properties of the $\gamma$-dependent spaces $H_{*, \gamma}^{m}\left(\mathbb{R}_{+}^{n}\right)$, that are used in this paper, are proved in Appendix Appendix B, where our main concern is to show that the a priori estimates of Section 6 do not explode but are uniformly controlled when $\gamma$ is taken sufficiently large.

\section{Preliminaries and technical tools}

In this Section, we collect several technical tools that will be used in the subsequent analysis (cf. the next Section 4).

We start by recalling the definition of two operators $\sharp$ and $\natural$, introduced by Nishitani and Takayama in [19], with the main property of mapping isometrically square integrable (resp. essentially bounded) functions over the half-space $\mathbb{R}_{+}^{n}$ onto square integrable (resp. essentially bounded) functions over the full space $\mathbb{R}^{n}$.

The mappings $\sharp: L^{2}\left(\mathbb{R}_{+}^{n}\right) \rightarrow L^{2}\left(\mathbb{R}^{n}\right)$ and $\sharp: L^{\infty}\left(\mathbb{R}_{+}^{n}\right) \rightarrow L^{\infty}\left(\mathbb{R}^{n}\right)$ are respectively defined by

$$
w^{\sharp}(x):=w\left(e^{x_{1}}, x^{\prime}\right) e^{x_{1} / 2}, \quad a^{\natural}(x)=a\left(e^{x_{1}}, x^{\prime}\right), \quad \forall x=\left(x_{1}, x^{\prime}\right) \in \mathbb{R}^{n} .
$$

They are both norm preserving bijections.

It is also useful to notice that the above operators can be extended to the set $\mathcal{D}^{\prime}\left(\mathbb{R}_{+}^{n}\right)$ of Schwartz distributions in $\mathbb{R}_{+}^{n}$. It is easily seen that both $\sharp$ and $\natural$ are topological isomorphisms of the space $C_{0}^{\infty}\left(\mathbb{R}_{+}^{n}\right)$ of test functions in $\mathbb{R}_{+}^{n}\left(\right.$ resp. $C^{\infty}\left(\mathbb{R}_{+}^{n}\right)$ ) onto 
the space $C_{0}^{\infty}\left(\mathbb{R}^{n}\right)$ of test functions in $\mathbb{R}^{n}$ (resp. $C^{\infty}\left(\mathbb{R}^{n}\right)$ ). Therefore, a standard duality argument yields that $\sharp$ and $\natural$ can be defined on $\mathcal{D}^{\prime}\left(\mathbb{R}_{+}^{n}\right)$ by

$$
\begin{aligned}
& \left\langle u^{\sharp}, \varphi\right\rangle:=\left\langle u, \varphi^{\sharp-1}\right\rangle, \\
& \left\langle u^{\natural}, \varphi\right\rangle:=\left\langle u, \varphi^{b}\right\rangle, \quad \forall \varphi \in C_{0}^{\infty}\left(\mathbb{R}^{n}\right)
\end{aligned}
$$

$(\langle\cdot, \cdot\rangle$ is used to denote the duality pairing between distributions and test functions either in the half-space $\mathbb{R}_{+}^{n}$ or the full space $\mathbb{R}^{n}$ ). In the right-hand sides of (3.1), $\sharp^{-1}$ is just the inverse operator of $\sharp$, while the operator $b$ is defined by

$$
\varphi^{b}(x)=\frac{1}{x_{1}} \varphi\left(\log x_{1}, x^{\prime}\right), \quad \forall x_{1}>0, x^{\prime} \in \mathbb{R}^{n-1},
$$

for functions $\varphi \in C_{0}^{\infty}\left(\mathbb{R}^{n}\right)$. The operators $\sharp^{-1}$ and $b$ arise by explicitly calculating the formal adjoints of $\sharp$ and $\downarrow$ respectively.

Of course, one has that $u^{\sharp}, u^{\natural} \in \mathcal{D}^{\prime}\left(\mathbb{R}^{n}\right)$; moreover the following relations can be easily verified (cf. [19])

$$
\begin{array}{r}
(\psi u)^{\sharp}=\psi^{\natural} u^{\sharp}, \\
\partial_{j}\left(u^{\natural}\right)=\left(Z_{j} u\right)^{\natural}, \quad j=1, \ldots, n, \\
\partial_{1}\left(u^{\sharp}\right)=\left(Z_{1} u\right)^{\sharp}+\frac{1}{2} u^{\sharp}, \\
\partial_{j}\left(u^{\sharp}\right)=\left(Z_{j} u\right)^{\sharp}, \quad j=2, \ldots, n,
\end{array}
$$

whenever $u \in \mathcal{D}^{\prime}\left(\mathbb{R}_{+}^{n}\right)$ and $\psi \in C^{\infty}\left(\mathbb{R}_{+}^{n}\right)$ (in (3.3) $u \in L^{2}\left(\mathbb{R}_{+}^{n}\right)$ and $\psi \in L^{\infty}\left(\mathbb{R}_{+}^{n}\right)$ are also allowed).

From formulas (3.5), (3.6) and the $L^{2}$-boundedness of $\sharp$, it also follows that $\sharp$ : $H_{t a n, \gamma}^{m}\left(\mathbb{R}_{+}^{n}\right) \rightarrow H_{\gamma}^{m}\left(\mathbb{R}^{n}\right)$ is a topological isomorphism, for each integer $m \geq 1$ and real $\gamma \geq 1$.

Following [19] (see also [18]), in the next Subsection the last property of $\sharp$ will be exploited to shift some remarkable properties of the ordinary Sobolev spaces in $\mathbb{R}^{n}$ to the functional framework of conormal Sobolev spaces over the half-space $\mathbb{R}_{+}^{n}$.

Let us denote by $C_{(0)}^{\infty}\left(\mathbb{R}_{+}^{n}\right)$ the set of restrictions to $\mathbb{R}_{+}^{n}$ of functions of $C_{0}^{\infty}\left(\mathbb{R}^{n}\right)$. In the end, we observe that the operator $\sharp$ continuously maps the space $C_{(0)}^{\infty}\left(\mathbb{R}_{+}^{n}\right)$ into the space $\mathcal{S}\left(\mathbb{R}^{n}\right)$ of rapidly decreasing functions in $\mathbb{R}^{n}$ (note also that the same is no longer true for the image of $C_{(0)}^{\infty}\left(\mathbb{R}_{+}^{n}\right)$ under the operator $\natural$, which is only included into the space $C_{b}^{\infty}\left(\mathbb{R}^{n}\right)$ of infinitely smooth functions in $\mathbb{R}^{n}$, with bounded derivatives of all orders).

\subsection{Parameter depending norms on Sobolev spaces}

We start by recalling a classical characterization of ordinary Sobolev spaces in $\mathbb{R}^{n}$, due to Hörmander [14], based upon the uniform boundedness of a suitable family of parameter-depending norms.

For given $s \in \mathbb{R}, \gamma \geq 1$ and for each $\delta \in] 0,1]$ a norm in $H^{s-1}\left(\mathbb{R}^{n}\right)$ is defined by 
setting

$$
\|u\|_{s-1, \gamma, \delta}^{2}:=(2 \pi)^{-n} \int_{\mathbb{R}^{n}} \lambda^{2 s, \gamma}(\xi) \lambda^{-2, \gamma}(\delta \xi)|\widehat{u}(\xi)|^{2} d \xi .
$$

According to Section 2 , for $\gamma=1$ and any $0<\delta \leq 1$ we set $\|\cdot\|_{s-1, \delta}:=\|\cdot\| \|_{s-1,1, \delta}$; the family of $\delta$-weighted norms $\left\{\|\cdot\|_{s-1, \delta}\right\}_{0<\delta \leq 1}$ was deeply studied in [14]; easy arguments (relying essentially on a $\gamma$-rescaling of functions) lead to get the same properties for the norms $\left\{\|\cdot\|_{s-1, \gamma, \delta}\right\}_{0<\delta \leq 1}$ defined in (3.7) with an arbitrary $\gamma \geq 1$.

Of course, one has $\|\cdot\|_{s-1, \gamma, 1}=\|\cdot\|_{s-1, \gamma}$ (cf. (2.2), with $s-1$ instead of $s$ ). It is also clear that, for each fixed $\delta \in] 0,1\left[\right.$, the norm $\|\cdot\|_{s-1, \gamma, \delta}$ is equivalent to $\|\cdot\|_{s-1, \gamma}$ in $H_{\gamma}^{s-1}\left(\mathbb{R}^{n}\right)$, uniformly with respect to $\gamma$; notice, however, that the constants appearing in the equivalence inequalities will generally depend on $\delta$ (see $(3.14))$.

The next characterization of Sobolev spaces readily follows by taking account of the parameter $\gamma$ into the arguments used in [14, Thm. 2.4.1].

Proposition 3.1. For every $s \in \mathbb{R}$ and $\gamma \geq 1, u \in H_{\gamma}^{s}\left(\mathbb{R}^{n}\right)$ if and only if $u \in$ $H_{\gamma}^{s-1}\left(\mathbb{R}^{n}\right)$, and the set $\left\{\|u\|_{s-1, \gamma, \delta}\right\}_{0<\delta \leq 1}$ is bounded. In this case, we have

$$
\|u\|_{s-1, \gamma, \delta} \uparrow\|u\|_{s, \gamma}, \quad \text { as } \delta \downarrow 0 .
$$

In order to show the regularity result stated in Theorem 4.1, it is useful providing the conormal Sobolev space $H_{t a n, \gamma}^{m-1}\left(\mathbb{R}_{+}^{n}\right), m \in \mathbb{N}, \gamma \geq 1$, with a family of parameterdepending norms satisfying analogous properties to that of norms defined in (3.7). Such norms were defined by Nishitani and Takayama [19], in the "unweighted" case $\gamma=1$, just applying the ordinary Sobolev norms $\|\cdot\|_{m-1, \delta}$ to pull-back of functions on $\mathbb{R}_{+}^{n}$, by the $\sharp$ operator; then these norms were used in [18] to characterize the conormal regularity of functions.

Following [19], for $\gamma \geq 1, \delta \in] 0,1]$ and all $u \in H_{\text {tan }}^{m-1}\left(\mathbb{R}_{+}^{n}\right)$ we set

$$
\|u\|_{\mathbb{R}_{+}^{n}, m-1, \tan , \gamma, \delta}^{2}:=\left\|u^{\sharp}\right\|_{m-1, \gamma, \delta}^{2}=(2 \pi)^{-n} \int_{\mathbb{R}^{n}} \lambda^{2 m, \gamma}(\xi) \lambda^{-2, \gamma}(\delta \xi)\left|\widehat{u^{\sharp}}(\xi)\right|^{2} d \xi .
$$

Because $\sharp$ is an isomorphism of $H_{\tan , \gamma}^{m-1}\left(\mathbb{R}_{+}^{n}\right)$ onto $H_{\gamma}^{m-1}\left(\mathbb{R}^{n}\right)$, the family of norms $\left\{\|\cdot\|_{\mathbb{R}_{+}^{n}, m-1, \tan , \gamma, \delta}\right\}_{0<\delta \leq 1}$ keeps all the properties enjoied by the family of norms defined in (3.7).

In particular, the same characterization of ordinary Sobolev spaces on $\mathbb{R}^{n}$, given by Proposition 3.1, applies also to conormal Sobolev spaces in $\mathbb{R}_{+}^{n}$ (cf. [19], [18]).

Proposition 3.2. For every positive integer $m$ and $\gamma \geq 1, u \in H_{\text {tan, } \gamma}^{m}\left(\mathbb{R}_{+}^{n}\right)$ if and only if $u \in H_{\text {tan, }, \gamma}^{m-1}\left(\mathbb{R}_{+}^{n}\right)$, and the set $\left\{\|u\|_{\mathbb{R}_{+}^{n}, m-1, \tan , \gamma, \delta}\right\}_{0<\delta \leq 1}$ is bounded. In this case, we have

$$
\|u\|_{\mathbb{R}_{+}^{n}, m-1, \tan , \gamma, \delta} \uparrow\|u\|_{\mathbb{R}_{+}^{n}, m, \tan , \gamma}, \quad \text { as } \delta \downarrow 0 .
$$




\subsection{A class of conormal operators}

The $\sharp$ operator, defined at the beginning of Section 3, can be used to allow pseudodifferential operators in $\mathbb{R}^{n}$ acting conormally on functions only defined over the positive half-space $\mathbb{R}_{+}^{n}$. Then the standard machinery of pseudo-differential calculus (in the parameter depending version well suited to hyperbolic problems, introduced in [1], [7]) can be re-arranged into a functional calculus properly behaved on conormal Sobolev spaces described in Section 2. In Section 4, this calculus will be usefully applied to study the conormal regularity of the stationary BVP (4.5).

Let us introduce the pseudo-differential symbols, with a parameter, to be used later; here we closely follow the terminology and notations of [8].

Definition 3.3. A parameter-depending pseudo-differential symbol of order $m \in \mathbb{R}$ is a real (or complex)-valued measurable function a $(x, \xi, \gamma)$ on $\mathbb{R}^{n} \times \mathbb{R}^{n} \times[1,+\infty[$, such that $a$ is $C^{\infty}$ with respect to $x$ and $\xi$ and for all multi-indices $\alpha, \beta \in \mathbb{N}^{n}$ there exists a positive constant $C_{\alpha, \beta}$ satisfying:

$$
\left|\partial_{\xi}^{\alpha} \partial_{x}^{\beta} a(x, \xi, \gamma)\right| \leq C_{\alpha, \beta} \lambda^{m-|\alpha|, \gamma}(\xi),
$$

for all $x, \xi \in \mathbb{R}^{n}$ and $\gamma \geq 1$.

The same definition as above extends to functions $a(x, \xi, \gamma)$ taking values in the space $\mathbb{R}^{N \times N}$ (resp. $\mathbb{C}^{N \times N}$ ) of $N \times N$ real (resp. complex)-valued matrices, for all integers $N>1$ (where the module $|\cdot|$ is replaced in (3.9) by any equivalent norm in $\mathbb{R}^{N \times N}$ (resp. $\left.\mathbb{C}^{N \times N}\right)$ ). We denote by $\Gamma^{m}$ the set of $\gamma$-depending symbols of order $m \in \mathbb{R}$ (the same notation being used for both scalar or matrix-valued symbols). $\Gamma^{m}$ is equipped with the obvious norms

$$
|a|_{m, k}:=\max _{|\alpha|+|\beta| \leq k} \sup _{(x, \xi) \in \mathbb{R}^{n} \times \mathbb{R}^{n}, \gamma \geq 1} \lambda^{-m+|\alpha|, \gamma}(\xi)\left|\partial_{\xi}^{\alpha} \partial_{x}^{\beta} a(x, \xi, \gamma)\right|, \quad \forall k \in \mathbb{N},
$$

which turn it into a Fréchet space. For all $m, m^{\prime} \in \mathbb{R}$, with $m \leq m^{\prime}$, the continuous imbedding $\Gamma^{m} \subset \Gamma^{m^{\prime}}$ can be easily proven.

For all $m \in \mathbb{R}$, the function $\lambda^{m, \gamma}$ is of course a (scalar-valued) symbol in $\Gamma^{m}$.

To perform the analysis of Section 4, it is important to consider the behavior of the weight function $\lambda^{m, \gamma} \lambda^{-1, \gamma}(\delta \cdot)$, involved in the definition of the parameterdepending norms in (3.7), (3.8), as a $\gamma$-depending symbol according to Definition 3.3 .

In order to simplify the forthcoming statements, henceforth the following short notations will be used

$$
\begin{aligned}
& \lambda_{\delta}^{m-1, \gamma}(\xi):=\lambda^{m, \gamma}(\xi) \lambda^{-1, \gamma}(\delta \xi), \\
& \tilde{\lambda}_{\delta}^{-m+1, \gamma}(\xi):=\left(\lambda_{\delta}^{m-1, \gamma}(\xi)\right)^{-1}\left(=\lambda^{-m, \gamma}(\xi) \lambda^{1, \gamma}(\delta \xi)\right),
\end{aligned}
$$

for all real numbers $m \in \mathbb{R}, \gamma \geq 1$ and $\delta \in] 0,1]$. One has the obvious identities $\lambda_{1}^{m-1, \gamma}(\xi) \equiv \lambda^{m-1, \gamma}(\xi), \widetilde{\lambda}_{1}^{-m+1, \gamma}(\xi) \equiv \lambda_{1}^{-m+1, \gamma}(\xi) \equiv \lambda^{-m+1, \gamma}(\xi)$. However, to avoid 
confusion in the following, we remark that functions $\lambda_{\delta}^{-m+1, \gamma}(\xi)$ and $\widetilde{\lambda}_{\delta}^{-m+1, \gamma}(\xi)$ are no longer the same as soon as $\delta$ becomes strictly smaller than 1 ; indeed (3.11) gives $\lambda_{\delta}^{-m+1, \gamma}(\xi)=\lambda^{-m+2, \gamma}(\xi) \lambda^{-1, \gamma}(\delta \xi)$.

A straightforward application of Leibniz's rule leads to the following result.

Lemma 3.4. For every $m \in \mathbb{R}$ and all $\alpha \in \mathbb{N}^{n}$ there exists a positive constant $C_{m, \alpha}$ such that

$$
\left.\left.\left|\partial_{\xi}^{\alpha} \lambda_{\delta}^{m-1, \gamma}(\xi)\right| \leq C_{m, \alpha} \lambda_{\delta}^{m-1-|\alpha|, \gamma}(\xi), \quad \forall \xi \in \mathbb{R}^{n}, \gamma \geq 1, \delta \in\right] 0,1\right] .
$$

Because of estimates $(3.12), \lambda_{\delta}^{m-1, \gamma}(\xi)$ can be regarded as a $\gamma$-depending symbol, in two different ways. On the one hand, combining estimates (3.12) with the trivial inequality

$$
\lambda^{-1, \gamma}(\delta \xi) \leq 1
$$

immediately gives that $\left\{\lambda_{\delta}^{m-1, \gamma}\right\}_{0<\delta \leq 1}$ is a bounded subset of $\Gamma^{m}$.

On the other hand, the left inequality in

$$
\left.\left.\delta \lambda^{1, \gamma}(\xi) \leq \lambda^{1, \gamma}(\delta \xi) \leq \lambda^{1, \gamma}(\xi), \quad \forall \xi \in \mathbb{R}^{n}, \forall \delta \in\right] 0,1\right],
$$

together with (3.12), also gives

$$
\left|\partial_{\xi}^{\alpha} \lambda_{\delta}^{m-1, \gamma}(\xi)\right| \leq C_{m, \alpha} \delta^{-1} \lambda^{m-1-|\alpha|, \gamma}(\xi), \quad \forall \xi \in \mathbb{R}^{n}, \gamma \geq 1 .
$$

According to Definition 3.3, (3.15) means that $\lambda_{\delta}^{m-1, \gamma}$ actually belongs to $\Gamma^{m-1}$ for each fixed $\delta$; nevertheless, the family $\left\{\lambda_{\delta}^{m-1, \gamma}\right\}_{0<\delta \leq 1}$ is generally unbounded as a subset of $\Gamma^{m-1}$.

For later use, we also need to study the behavior of functions $\widetilde{\lambda}_{\delta}^{-m+1, \gamma}$ as $\gamma$-depending symbols.

Analogously to Lemma 3.4, one can prove the following result.

Lemma 3.5. For all $m \in \mathbb{R}$ and $\alpha \in \mathbb{N}^{n}$ there exists $\widetilde{C}_{m, \alpha}>0$ such that

$$
\left.\left.\left|\partial_{\xi}^{\alpha} \widetilde{\lambda}_{\delta}^{-m+1, \gamma}(\xi)\right| \leq \widetilde{C}_{m, \alpha} \widetilde{\lambda}_{\delta}^{-m+1-|\alpha|, \gamma}(\xi), \quad \forall \xi \in \mathbb{R}^{n}, \gamma \geq 1, \delta \in\right] 0,1\right] .
$$

In particular, Lemma 3.5 implies that the family $\left\{\widetilde{\lambda}_{\delta}^{-m+1, \gamma}\right\}_{0<\delta \leq 1}$ is a bounded subset of $\Gamma^{-m+1}$ (it suffices to combine (3.16) with the right inequality in (3.14)).

Any symbol $a=a(x, \xi, \gamma) \in \Gamma^{m}$ defines a pseudo-differential operator $\operatorname{Op}^{\gamma}(a)=$ $a(x, D, \gamma)$ on the Schwartz space $\mathcal{S}\left(\mathbb{R}^{n}\right)$, by the standard formula

$$
\begin{aligned}
& \forall u \in \mathcal{S}\left(\mathbb{R}^{n}\right), \forall x \in \mathbb{R}^{n}, \\
& \operatorname{Op}^{\gamma}(a) u(x)=a(x, D, \gamma) u(x)=:(2 \pi)^{-n} \int_{\mathbb{R}^{n}} e^{i x \cdot \xi} a(x, \xi, \gamma) \widehat{u}(\xi) d \xi,
\end{aligned}
$$

where, of course, we denote $x \cdot \xi:=\sum_{j=1}^{n} x_{j} \xi_{j} . \mathrm{Op}^{\gamma}(a)$ is called the pseudo-differential operator with symbol $a ; m$ is the order of $\operatorname{Op}^{\gamma}(a)$. It comes from the classical theory that $\mathrm{Op}^{\gamma}(a)$ defines a linear bounded operator

$$
\operatorname{Op}^{\gamma}(a): \mathcal{S}\left(\mathbb{R}^{n}\right) \rightarrow \mathcal{S}\left(\mathbb{R}^{n}\right)
$$


moreover, the latter extends to a linear bounded operator on the space $\mathcal{S}^{\prime}\left(\mathbb{R}^{n}\right)$ of tempered distributions in $\mathbb{R}^{n}$. An exhaustive account of the symbolic calculus for pseudo-differential operators with symbols in $\Gamma^{m}$ can be found in [7]. Here, we just recall the following result, concerning the product and the commutator of two pseudo-differential operators.

Proposition 3.6. Let $a \in \Gamma^{m}$ and $b \in \Gamma^{l}$, for $l, m \in \mathbb{R}$. Then $\operatorname{Op}^{\gamma}(a) \operatorname{Op}^{\gamma}(b)$ is a pseudo-differential operator with symbol in $\Gamma^{m+l}$; moreover, if we let a\#b denote the symbol of the product, one has for every integer $N \geq 1$

$$
a \# b-\sum_{|\alpha|<N} \frac{(-i)^{|\alpha|}}{\alpha !} \partial_{\xi}^{\alpha} a \partial_{x}^{\alpha} b \in \Gamma^{m+l-N} .
$$

Under the same assumptions, the commutator $\left[\mathrm{Op}^{\gamma}(a), \mathrm{Op}^{\gamma}(b)\right]:=\mathrm{Op}^{\gamma}(a) \mathrm{Op}^{\gamma}(b)-$ $\mathrm{Op}^{\gamma}(b) \mathrm{Op}^{\gamma}(a)$ is again a pseudo-differential operator with symbol $c \in \Gamma^{m+l}$. If we further assume that one of the two symbols $a$ or $b$ is scalar-valued (so that $a$ and $b$ commute in the product), then the symbol c of $\left[\mathrm{Op}^{\gamma}(a), \mathrm{Op}^{\gamma}(b)\right]$ has order $m+l-1$.

We point out that when the symbol $b \in \Gamma^{l}$ of the preceding statement does not depend on the $x$ variables (i.e. $b=b(\xi, \gamma)$ ) then the symbol $a \# b$ of the product $\mathrm{Op}^{\gamma}(a) \mathrm{Op}^{\gamma}(b)$ reduces to the point-wise product of symbols $a$ and $b$; in this case, the asymptotic formula (3.18) is replaced by the exact formula

$$
(a \# b)(x, \xi, \gamma)=a(x, \xi, \gamma) b(\xi, \gamma) .
$$

According to (3.11), (3.17), we write:

$$
\lambda_{\delta}^{m-1, \gamma}(D):=\operatorname{Op}^{\gamma}\left(\lambda_{\delta}^{m-1, \gamma}\right), \quad \widetilde{\lambda}_{\delta}^{-m+1, \gamma}(D):=\operatorname{Op}^{\gamma}\left(\widetilde{\lambda}_{\delta}^{-m+1, \gamma}\right) .
$$

In view of (3.11) and (3.19), the operator $\lambda_{\delta}^{m-1, \gamma}(D)$ is invertible, and its two-sided inverse is given by $\widetilde{\lambda}_{\delta}^{-m+1, \gamma}(D)$.

Starting from the symbolic classes $\Gamma^{m}, m \in \mathbb{R}$, we introduce now the class of conormal operators in $\mathbb{R}_{+}^{n}$, to be used in the sequel.

Definition 3.7. Let $a(x, \xi, \gamma)$ be a $\gamma$-depending symbol in $\Gamma^{m}, m \in \mathbb{R}$. The conormal operator with symbol a, denoted by $\mathrm{Op}_{\sharp}^{\gamma}(a)$ (or equivalently $a(x, Z, \gamma)$ ) is defined by setting

$$
\forall u \in C_{(0)}^{\infty}\left(\mathbb{R}_{+}^{n}\right), \quad\left(\mathrm{Op}_{\sharp}^{\gamma}(a) u\right)^{\sharp}=\left(\mathrm{Op}^{\gamma}(a)\right)\left(u^{\sharp}\right) .
$$

In other words, the operator $\mathrm{Op}_{\sharp}^{\gamma}(a)$ is the composition of mappings

$$
\mathrm{Op}_{\sharp}^{\gamma}(a)=\sharp^{-1} \circ \mathrm{Op}^{\gamma}(a) \circ \sharp \text {. }
$$

As we already noted, $u^{\sharp} \in \mathcal{S}\left(\mathbb{R}^{n}\right)$ whenever $u \in C_{(0)}^{\infty}\left(\mathbb{R}_{+}^{n}\right)$; hence formula (3.21) makes sense and gives that $\mathrm{Op}_{\sharp}^{\gamma}(a) u$ is a $C^{\infty}$-function in $\mathbb{R}_{+}^{n}$. Also $\mathrm{Op}_{\sharp}^{\gamma}(a)$ : $C_{(0)}^{\infty}\left(\mathbb{R}_{+}^{n}\right) \rightarrow C^{\infty}\left(\mathbb{R}_{+}^{n}\right)$ is a linear bounded operator that extends to a linear bounded operator from the space of distributions $u \in \mathcal{D}^{\prime}\left(\mathbb{R}_{+}^{n}\right)$ satisfying $u^{\sharp} \in \mathcal{S}^{\prime}\left(\mathbb{R}^{n}\right)$ into 
$\mathcal{D}^{\prime}\left(\mathbb{R}_{+}^{n}\right)$ itself $^{\text {a }}$. Throughout the paper, we continue to denote this extension by $\mathrm{Op}_{\sharp}^{\gamma}(a)$ (or $a(x, Z, \gamma)$ equivalently).

As an immediate consequence of (3.22), we have that for all symbols $a \in \Gamma^{m}, b \in \Gamma^{l}$, with $m, l \in \mathbb{R}$, there holds

$$
\forall u \in C_{(0)}^{\infty}\left(\mathbb{R}_{+}^{n}\right), \quad \mathrm{Op}_{\sharp}^{\gamma}(a) \mathrm{Op}_{\sharp}^{\gamma}(b) u=\left(\mathrm{Op}^{\gamma}(a) \mathrm{Op}^{\gamma}(b)\left(u^{\sharp}\right)\right)^{\sharp^{-1}} .
$$

Then, it is clear that a functional calculus of conormal operators can be straightforwardly borrowed from the pseudo-differential calculus in $\mathbb{R}^{n}$; in particular we find that products and commutators of conormal operators are still operators of the same type, and their symbols are computed according to the rules collected in Proposition 3.6.

Below, let us consider the main examples of conormal operators that will be met in Section 4.

As a first example, we quote the multiplication by a matrix-valued function $B \in C_{(0)}^{\infty}\left(\mathbb{R}_{+}^{n}\right)$. It is clear that this makes an operator of order zero according to (3.21); indeed (3.3) gives for any vector-valued $u \in C_{(0)}^{\infty}\left(\mathbb{R}_{+}^{n}\right)$

$$
(B u)^{\sharp}(x)=B^{\natural}(x) u^{\sharp}(x),
$$

and $B^{\natural}$ is a $C^{\infty}$-function in $\mathbb{R}^{n}$, with bounded derivatives of any order, hence a symbol in $\Gamma^{0}$. We remark that, when computed for $B^{\natural}$, the norm of order $k \in \mathbb{N}$, defined on symbols by (3.10), just reduces to

$$
\left|B^{\natural}\right|_{0, k}=\max _{|\alpha| \leq k}\left\|\partial^{\alpha} B^{\natural}\right\|_{L^{\infty}\left(\mathbb{R}^{n}\right)}=\max _{|\alpha| \leq k} \|\left. Z^{\alpha} B\right|_{L^{\infty}\left(\mathbb{R}_{+}^{n}\right)},
$$

where the second identity above exploits formulas (3.4) and that $\downarrow$ maps isometrically $L^{\infty}\left(\mathbb{R}_{+}^{n}\right)$ onto $L^{\infty}\left(\mathbb{R}^{n}\right)$.

Now, let $\mathcal{L}:=\gamma I_{N}+\sum_{j=1}^{n} A_{j}(x) Z_{j}$ be a first order linear differential operator, with matrix-valued coefficients $A_{j} \in C_{(0)}^{\infty}\left(\mathbb{R}_{+}^{n}\right)$ for $j=1, \ldots, n$ and $\gamma \geq 1$. Since the leading part of $\mathcal{L}$ only involves conormal derivatives, applying (3.3), (3.5), (3.6) then gives

$$
\left(\gamma u+\sum_{j=1}^{n} A_{j} Z_{j} u\right)^{\sharp}=\left(\gamma I-\frac{1}{2} A_{1}^{\natural}\right) u^{\sharp}+\sum_{j=1}^{n} A_{j}^{\natural} \partial_{j} u^{\sharp}=\operatorname{Op}^{\gamma}(a) u^{\sharp},
$$

where $a=a(x, \xi, \gamma):=\left(\gamma I_{N}-\frac{1}{2} A_{1}^{\natural}(x)\right)+i \sum_{j=1}^{n} A_{j}^{\natural}(x) \xi_{j}$ is a symbol in $\Gamma^{1}$. Then $\mathcal{L}$ is a conormal operator of order 1 , according to (3.21).

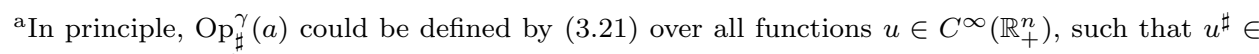
$\mathcal{S}\left(\mathbb{R}^{n}\right)$. Then $\mathrm{Op}_{\sharp}^{\gamma}(a)$ defines a linear bounded operator from the latter function space into itself, provided we equip this space with the topology induced, via $\sharp$, from the Fréchet topology of $\mathcal{S}\left(\mathbb{R}^{n}\right)$. 
In the next Section 4, we will be mainly interested to the family of conormal operators

$$
\lambda_{\delta}^{m-1, \gamma}(Z):=\mathrm{Op}_{\sharp}^{\gamma}\left(\lambda_{\delta}^{m-1, \gamma}\right), \quad \tilde{\lambda}_{\delta}^{-m+1, \gamma}(Z):=\mathrm{Op}_{\sharp}^{\gamma}\left(\widetilde{\lambda}_{\delta}^{-m+1, \gamma}\right) .
$$

The operators $\lambda_{\delta}^{m-1, \gamma}(Z)$ are involved in the characterization of conormal regularity provided by Proposition 3.2 (remember that, after Lemma 3.4, $\lambda_{\delta}^{m-1, \gamma} \in \Gamma^{m-1}$ ). Indeed, from Plancherel's formula and the fact that the operator $\sharp$ preserves the $L^{2}$-norm, the following identities

$$
\|u\|_{\mathbb{R}_{+}^{n}, m-1, \tan , \gamma, \delta} \equiv\left\|\lambda_{\delta}^{m-1, \gamma}(Z) u\right\|_{L^{2}\left(\mathbb{R}_{+}^{n}\right)}
$$

can be straightforwardly established; hence, Proposition 3.2 can be restated in terms of the boundedness, with respect to $\delta$, of the $L^{2}$-norms of functions $\lambda_{\delta}^{m-1, \gamma}(Z) u$. This observation is the key point that leads to the analysis performed in Section 4 . Another main feature of the conormal operators $(3.26)$ is that $\widetilde{\lambda}_{\delta}^{-m+1, \gamma}(Z)$ provides a two-sided inverse of $\lambda_{\delta}^{m-1, \gamma}(Z)$; this comes at once from the analogous property of the operators in (3.20) and formulas (3.21), (3.23).

\subsection{Sobolev continuity of conormal operators}

Proposition 3.8. If $s, m \in \mathbb{R}$ then for all $a \in \Gamma^{m}$ the pseudo-differential operator $\mathrm{Op}^{\gamma}(a)$ extends as a linear bounded operator from $H_{\gamma}^{s+m}\left(\mathbb{R}^{n}\right)$ into $H_{\gamma}^{s}\left(\mathbb{R}^{n}\right)$, and the operator norm of such an extension is uniformly bounded with respect to $\gamma$.

We refer the reader to [7] for a detailed proof of Proposition 3.8; a sharp calculation shows that the norm of $\mathrm{Op}^{\gamma}(a)$, as a linear bounded operator from $H_{\gamma}^{s+m}\left(\mathbb{R}^{n}\right)$ to $H_{\gamma}^{s}\left(\mathbb{R}^{n}\right)$, actually depends only on a norm of type (3.10) of the symbol $a$, besides the Sobolev order $s$ and the symbolic order $m$ (cf. [7] for detailed calculations). This observation entails, in particular, that the operator norm is uniformly bounded with respect to $\gamma$ and other additional parameters from which the symbol of the operator should possibly depend, as a bounded map.

Using Proposition 3.8 and that the operator $\sharp$ maps isomorphically conormal Sobolev spaces on $\mathbb{R}_{+}^{n}$ to ordinary Sobolev spaces on $\mathbb{R}^{n}$, we easily derive the following result.

Proposition 3.9. If $m \in \mathbb{Z}$ and $a \in \Gamma^{m}$, then the conormal operator $\operatorname{Op}_{\sharp}^{\gamma}(a)$ extends to a linear bounded operator from $H_{\text {tan }, \gamma}^{s+m}\left(\mathbb{R}_{+}^{n}\right)$ to $H_{\text {tan }, \gamma}^{s}\left(\mathbb{R}_{+}^{n}\right)$, for every integer $s \geq 0$, such that $s+m \geq 0$; moreover the operator norm of such an extension is uniformly bounded with respect to $\gamma$.

Remark 3.10. We point out that, compared to Proposition 3.8, the statement above only deals with integer orders of symbols and conormal Sobolev spaces. The reason is that, in Section 2, conormal Sobolev spaces were only defined for positive integer orders. In principle, this lack could be removed by extending the definition of conormal spaces $H_{\text {tan }}^{s}\left(\mathbb{R}_{+}^{n}\right)$ to any real order $s$ : this could be trivially done, just 
defining $H_{\text {tan }}^{s}\left(\mathbb{R}_{+}^{n}\right)$ to be the pull-back, by the operator $\sharp$, of functions in $H^{s}\left(\mathbb{R}^{n}\right)$. However, this extension to fractional exponents seems to be useless for the subsequent developments.

\section{The homogeneous IBVP. Tangential regularity}

We introduce the new unknown $u_{\gamma}(x, t):=e^{-\gamma t} u(x, t)$ and the new data $F_{\gamma}:=$ $e^{-\gamma t} F(x, t), G_{\gamma}:=e^{-\gamma t} G(x, t)$. Then problem (1.1)-(1.3) becomes equivalent to

$$
\begin{array}{ll}
L_{\gamma} u_{\gamma}=F_{\gamma}, & \text { in } Q_{T}, \\
M u_{\gamma}=G_{\gamma}, & \text { on } \Sigma_{T} \\
u_{\gamma \mid t=0}=f, & \text { in } \mathbb{R}_{+}^{n},
\end{array}
$$

with

$$
L_{\gamma}:=\gamma+L
$$

In this section we concentrate on the study of the tangential regularity of the solution to the IBVP (4.1), where the initial datum $f$ is identically zero and the data $F_{\gamma}$ and $G_{\gamma}$ satisfy the compatibility conditions in a more restrictive form than (1.12). More precisely, we concentrate on the homogeneous IBVP

$$
\begin{array}{ll}
L_{\gamma} u_{\gamma}=F_{\gamma}, & \text { in } Q_{T}, \\
M u_{\gamma}=G_{\gamma}, & \text { on } \Sigma_{T}, \\
u_{\gamma \mid t=0}=0, & \text { in } \mathbb{R}_{+}^{n} .
\end{array}
$$

We remark that here and in the following the word homogeneous is referred by convention to the initial datum $f$.

For a given integer $m \geq 1$, we assume that $F_{\gamma}$ and $G_{\gamma}$ satisfy the following conditions

$$
\partial_{t}^{h} F_{\gamma \mid t=0}=0, \quad \partial_{t}^{h} G_{\gamma \mid t=0}=0, \quad h=0, \ldots, m .
$$

It is worth to notice that conditions (4.3) imply the compatibility conditions (1.12), in the case $f=0$. We prove the following theorem for smooth coefficients. The general case with coefficients of finite regularity will be treated later on by a density argument.

Theorem 4.1. Assume that $S_{0}, A_{i}, B$, for $i=1, \ldots, n$, are in $C_{(0)}^{\infty}(Q)$, and that problem (4.2) satisfies assumptions (1)-(4); then for all $T>0$ and $m \in \mathbb{N}$ there exist constants $C_{m}>0$ and $\gamma_{m}$, with $\gamma_{m} \geq \gamma_{m-1}$, such that for all $\gamma \geq \gamma_{m}$, $F_{\gamma} \in H_{t a n, \gamma}^{m+1}\left(Q_{T}\right)$ and $G_{\gamma} \in H_{\gamma}^{m+1}\left(\Sigma_{T}\right)$ satisfying (4.3) the unique solution $u_{\gamma}$ to (4.2) belongs to $H_{t a n, \gamma}^{m}\left(Q_{T}\right)$, the trace of $u_{\gamma}^{I}$ on $\Sigma_{T}$ belongs to $H_{\gamma}^{m}\left(\Sigma_{T}\right)$, and the a priori estimate

$\gamma\left\|u_{\gamma}\right\|_{H_{t a n, \gamma}^{m}\left(Q_{T}\right)}^{2}+\left\|u_{\gamma \mid \Sigma_{T}}^{I}\right\|_{H_{\gamma}^{m}\left(\Sigma_{T}\right)}^{2} \leq C_{m}\left(\frac{1}{\gamma^{3}}\left\|F_{\gamma}\right\|_{H_{t a n, \gamma}^{m+1}\left(Q_{T}\right)}^{2}+\frac{1}{\gamma^{2}}\left\|G_{\gamma}\right\|_{H_{\gamma}^{m+1}\left(\Sigma_{T}\right)}^{2}\right)$

is fulfilled. 
The first step to prove Theorem 4.1 is reducing the original problem (4.2) to a boundary value problem where the time is allowed to span the whole real line and is treated, consequently, as an additional tangential variable. To make this reduction, we extend the data $F_{\gamma}, G_{\gamma}$ and the unknown $u_{\gamma}$ of (4.2) to all positive and negative times. In the sequel, for the sake of simplicity, we remove the subscript $\gamma$ from the unknown $u_{\gamma}$ and the data $F_{\gamma}, G_{\gamma}$.

Because of conditions (4.3), we may extend $F, G$ by setting them equal to zero for all negative times and for $t>T$ by "reflection", so that the extended $F$ and $G$ vanish also for all $t>T$ sufficiently large. We get $F \in H_{t a n, \gamma}^{m+1}(Q)$ and $G \in H_{\gamma}^{m+1}(\Sigma)$. As we did for the data, the solution $u$ to (4.2) is extended to all negative times, by setting it equal to zero. We extend $u$ also for times $t>T$, by following the argument of [18], where we make use of assumption (4). By construction, $u$ solves the BVP

$$
\begin{array}{ll}
L_{\gamma} u=F, & \text { in } Q, \\
M u=G, & \text { on } \Sigma .
\end{array}
$$

In (4.5), the time $t$ is involved with the same role of the tangential space variables, as it spans the whole real line $\mathbb{R}$. Therefore, (4.5) is now a stationary problem posed in $Q$, with data $F \in H_{t a n, \gamma}^{m+1}(Q), G \in H_{\gamma}^{m+1}(\Sigma)$. Furthermore, $u$ enjoys the estimate (1.10), that is

$$
\gamma\|u\|_{L^{2}(Q)}^{2}+\left\|u_{\mid \Sigma}^{I}\right\|_{L^{2}(\Sigma)}^{2} \leq C_{0}^{\prime}\left(\frac{1}{\gamma^{3}}\|F\|_{H_{t a n, \gamma}^{1}(Q)}^{2}+\frac{1}{\gamma^{2}}\|G\|_{H_{\gamma}^{1}(\Sigma)}^{2}\right),
$$

for all $\gamma \geq \gamma_{0}^{\prime}$.

The proof of Theorem 4.1 will be derived as a consequence of the tangential regularity of solutions to the BVP (4.5). Thus we concentrate from now on this problem. It will be convenient to recover the notations $x_{n+1}:=t$ and $x:=\left(x_{1}, x^{\prime}, x_{n+1}\right)$ and denote $A_{n+1}=I, Z_{n+1}=\partial_{t}$.

We argue by induction on the integer order $m \geq 1$.

Let us take arbitrary data $F \in H_{t a n, \gamma}^{m+1}(Q), G \in H_{\gamma}^{m+1}(\Sigma)$. Because of the inductive hypothesis, we already know that the unique $L^{2}$-solution $u$ to (4.5) actually belongs to $H_{t a n, \gamma}^{m-1}(Q)$, and its trace on the boundary $u_{\mid x_{1}=0}^{I}$ belongs to $H_{\gamma}^{m-1}(\Sigma)$, provided that $\gamma$ is taken large enough; moreover the solution $u$ obeys the estimate of order $m-1$

$$
\gamma\|u\|_{H_{t a n, \gamma}^{m-1}(Q)}^{2}+\left\|u_{\mid x_{1}=0}^{I}\right\|_{H_{\gamma}^{m-1}(\Sigma)}^{2} \leq C_{m-1}\left(\frac{1}{\gamma^{3}}\|F\|_{H_{t a n, \gamma}^{m}(Q)}^{2}+\frac{1}{\gamma^{2}}\|G\|_{H_{\gamma}^{m}(\Sigma)}^{2}\right),
$$

where the positive constant $C_{m-1}$ only depends on $m, \mu$, and the $L^{\infty}$-norm of a finite number (depending on $m$ itself) of derivatives of $B$ (cf. (3.25)), besides the coefficients $A_{j}(1 \leq j \leq n)$ of $L$.

\subsection{The modified conormal Fourier multiplier}

In order to increase the conormal regularity of the solution $u$ by order one, we are going to act on the solution $u$ of the BVP (4.5) by the conormal operator $\lambda_{\delta}^{m-1, \gamma}(Z)$; 
then we will consider the problem satisfied by $\lambda_{\delta}^{m-1, \gamma}(Z) u$. However, due to some technical reasons that will be clarified in the next Section 4.2 , we need to slightly modify the conormal operator $\lambda_{\delta}^{m-1, \gamma}(Z)$.

The first step is to decompose the weight function $\lambda_{\delta}^{m-1, \gamma}$ as the sum of two contributions. To do so, we proceed as follows ${ }^{\mathrm{b}}$. Firstly, let us take an arbitrary positive, even function $\chi \in C_{0}^{\infty}\left(\mathbb{R}^{n+1}\right)$ with the following properties

$$
0 \leq \chi(x) \leq 1, \quad \forall x \in \mathbb{R}^{n+1}, \quad \chi(x) \equiv 1, \text { for }|x| \leq 1 / 2, \quad \chi(x) \equiv 0, \text { for }|x|>1 .
$$

Then, we set:

$$
\begin{aligned}
& \lambda_{\chi, \delta}^{m-1, \gamma}(\xi):=\chi(D)\left(\lambda_{\delta}^{m-1, \gamma}\right)(\xi)=\left(\mathcal{F}^{-1} \chi * \lambda_{\delta}^{m-1, \gamma}\right)(\xi), \\
& r_{m, \delta}(\xi, \gamma):=\lambda_{\delta}^{m-1, \gamma}(\xi)-\lambda_{\chi, \delta}^{m-1, \gamma}(\xi)=(I-\chi(D))\left(\lambda_{\delta}^{m-1, \gamma}\right)(\xi) .
\end{aligned}
$$

The following result (whose proof is given in [17]) shows that the function $\lambda_{\chi, \delta}^{m-1, \gamma}$ essentially behaves like $\lambda_{\delta}^{m-1, \gamma}$.

Lemma 4.2. Let the function $\chi \in C_{0}^{\infty}\left(\mathbb{R}^{n+1}\right)$ satisfy the assumptions in (4.8). Then $\lambda_{\chi, \delta}^{m-1, \gamma}$ is a symbol in $\Gamma^{m-1}$; moreover for every multi-index $\alpha \in \mathbb{N}^{n+1}$ there exists a positive constant $C_{m, \alpha}$, independent of $\gamma$ and $\delta$, such that

$$
\left|\partial_{\xi}^{\alpha} \lambda_{\chi, \delta}^{m-1, \gamma}(\xi)\right| \leq C_{m, \alpha} \lambda_{\delta}^{m-1-|\alpha|, \gamma}(\xi), \quad \forall \xi \in \mathbb{R}^{n+1} .
$$

An immediate consequence of Lemma 4.2 and (4.9) is that $r_{m, \delta}$ is also a $\gamma$-depending symbol in $\Gamma^{m-1}$.

Let us define, with the obvious meaning of the notations:

$$
\begin{array}{ll}
\lambda_{\chi, \delta}^{m-1, \gamma}(D):=\operatorname{Op}^{\gamma}\left(\lambda_{\chi, \delta}^{m-1, \gamma}\right), & r_{m, \delta}(D, \gamma):=\operatorname{Op}^{\gamma}\left(r_{m, \delta}\right), \\
\lambda_{\chi, \delta}^{m-1, \gamma}(Z):=\operatorname{Op}_{\sharp}^{\gamma}\left(\lambda_{\chi, \delta}^{m-1, \gamma}\right), & r_{m, \delta}(Z, \gamma):=\operatorname{Op}_{\sharp}^{\gamma}\left(r_{m, \delta}\right) .
\end{array}
$$

The second important result is concerned with the conormal operator $r_{m, \delta}(Z, \gamma)=$ $\mathrm{Op}_{\sharp}^{\gamma}\left(r_{m, \delta}\right)$, and tells that it essentially behaves as a regularizing operator on conormal Sobolev spaces.

Lemma 4.3. For every $k \in \mathbb{N}$, the conormal operator $r_{m, \delta}(Z, \gamma)$ extends as a linear bounded operator, still denoted by $r_{m, \delta}(Z, \gamma)$, from $L^{2}(Q)$ to $H_{\text {tan, },}^{k}(Q)$. Moreover there exists a positive constant $C_{m, k}$, depending only on $k$ and $m$, such that for all $\gamma \geq 1$ and $\delta \in] 0,1]$

$$
\left\|r_{m, \delta}(Z, \gamma) u\right\|_{H_{t a n, \gamma}^{k}(Q)} \leq C_{m, k} \gamma^{k}\|u\|_{L^{2}(Q)}, \quad \forall u \in L^{2}(Q) .
$$

The proof of Lemma 4.3 is given in [17]. According to (4.9), we decompose

$$
\lambda_{\delta}^{m-1, \gamma}(Z)=\lambda_{\chi, \delta}^{m-1, \gamma}(Z)+r_{m, \delta}(Z, \gamma) .
$$

${ }^{\mathrm{b}}$ From now on, for all the rest of Section 4, we will use the tools introduced in Section 3 where, due to the use of the time variable $t$ as an additional space variable, the dimension $n$ will be substituted by $n+1$. 
As a consequence of Lemmata 4.2, 4.3, the role of the family of conormal operators $\left\{\lambda_{\delta}^{m-1, \gamma}(Z)\right\}_{0<\delta \leq 1}$ in the characterization of the conormal regularity provided by Proposition 3.2 and (3.27) can also be played by the family of "modified" operators $\left\{\lambda_{\chi, \delta}^{m-1, \gamma}(Z)\right\}_{0<\delta \leq 1}$, namely we have the following

Corollary 4.4. For every positive integer $m$ and $\gamma \geq 1, u \in H_{t a n, \gamma}^{m}(Q)$ if and only if $u \in H_{\text {tan, } \gamma}^{m-1}(Q)$, and the set $\left\{\left\|\lambda_{\chi, \delta}^{m-1, \gamma}(Z) u\right\|_{L^{2}(Q)}\right\}_{0<\delta \leq 1}$ is bounded.

In order to suitably handle the commutator between the differential operator $L$ and the conormal operator $\lambda_{\chi, \delta}^{m-1, \gamma}(Z)$, that comes from deriving the problem satisfied by $\lambda_{\chi, \delta}^{m-1, \gamma}(Z) u$ (see Subsections 4.2.1,4.2.2), it is useful to analyze the behavior of the pseudo-differential operators $\lambda_{\chi, \delta}^{m-1, \gamma}(D)$, when interacting with another pseudodifferential operator by composition and commutation. The following Lemma analyzes these situations; actually here the function $\lambda_{\chi, \delta}^{m-1, \gamma}$ is replaced by a more general symbol $a_{\delta}$ preserving the same kind of decay properties as in (4.10).

Lemma 4.5. Let $\left\{a_{\delta}\right\}_{0<\delta \leq 1}$ be a family of symbols $a_{\delta}=a_{\delta}(x, \xi, \gamma) \in \Gamma^{r-1}, r \in \mathbb{R}$, such that for all multi-indices $\alpha, \beta \in \mathbb{N}^{n+1}$ there exists a positive constant $C_{r, \alpha, \beta}$, independent of $\gamma$ and $\delta$, for which:

$$
\left|\partial_{\xi}^{\alpha} \partial_{x}^{\beta} a_{\delta}(x, \xi, \gamma)\right| \leq C_{r, \alpha, \beta} \lambda_{\delta}^{r-1-|\alpha|, \gamma}(\xi), \quad \forall x, \xi \in \mathbb{R}^{n+1} .
$$

Let $b=b(x, \xi, \gamma)$ be another symbol in $\Gamma^{l}$, for $l \in \mathbb{R}$.

Then, for every $\delta \in] 0,1]$ the product $\mathrm{Op}^{\gamma}\left(a_{\delta}\right) \mathrm{Op}^{\gamma}(b)$ is a pseudo-differential operator with symbol $a_{\delta} \# b$ in $\Gamma^{l+r-1}$. Moreover, for all multi-indices $\alpha, \beta \in \mathbb{N}^{n+1}$ there exists a constant $C_{r, l, \alpha, \beta}$, independent of $\gamma$ and $\delta$, such that

$$
\left|\partial_{\xi}^{\alpha} \partial_{x}^{\beta}\left(a_{\delta} \# b\right)(x, \xi, \gamma)\right| \leq C_{r, l, \alpha, \beta} \lambda_{\delta}^{l+r-1-|\alpha|, \gamma}(\xi), \quad \forall x, \xi \in \mathbb{R}^{n+1} .
$$

Under the same hypotheses, $\mathrm{Op}^{\gamma}\left(a_{\delta}\right) \mathrm{Op}^{\gamma}(b) \widetilde{\lambda}_{\delta}^{-m+1, \gamma}(D)$ is a pseudo-differential operator with symbol $\left(a_{\delta} \# b\right) \widetilde{\lambda}_{\delta}^{-m+1, \gamma}$ in $\Gamma^{l+r-m}$; moreover, $\left\{\left(a_{\delta} \# b\right) \widetilde{\lambda}_{\delta}^{-m+1, \gamma}\right\}_{0<\delta \leq 1}$ is a bounded subset of $\Gamma^{l+r-m}$. Eventually, if the symbols $a_{\delta}$ are scalar-valued, $\left[\mathrm{Op}^{\gamma}\left(a_{\delta}\right), \mathrm{Op}^{\gamma}(b)\right] \widetilde{\lambda}_{\delta}^{-m+1, \gamma}(D)$ is a pseudo-differential operator with symbol $c_{\delta} \in$ $\Gamma^{l+r-m-1}$, and $\left\{c_{\delta}\right\}_{0<\delta \leq 1}$ is a bounded subset of $\Gamma^{l+r-m-1}$.

The proof of Lemma 4.5 is given in [17].

Remark 4.6. The fact that the symbols of $\mathrm{Op}^{\gamma}\left(a_{\delta}\right) \mathrm{Op}^{\gamma}(b)$, $\mathrm{Op}^{\gamma}\left(a_{\delta}\right) \mathrm{Op}^{\gamma}(b) \widetilde{\lambda}_{\delta}^{-m+1, \gamma}(D)$ and the symbol of $\left[\mathrm{Op}^{\gamma}\left(a_{\delta}\right), \mathrm{Op}^{\gamma}(b)\right] \widetilde{\lambda}_{\delta}^{-m+1, \gamma}(D)$ belong respectively to $\Gamma^{l+r-1}, \Gamma^{l+r-m}$ and $\Gamma^{l+r-m-1}$ (for scalar-valued $a_{\delta}$ ) follows at once from the standard rules of symbolic calculus summarized in Proposition 3.6. The non trivial part of the statement above (although deduced from the asymptotic formula (3.18) with a minor effort) is the one asserting that the symbol of $\mathrm{Op}^{\gamma}\left(a_{\delta}\right) \mathrm{Op}^{\gamma}(b)$ enjoys estimates (4.15); indeed, it gives the precise dependence on $\delta$ of the decay at infinity of this symbol. Then the remaining assertions in Lemma 4.5 easily follow from (4.15) itself. 
Remark 4.7. In view of Proposition 3.9, the results on symbols collected in Lemma 4.5 can be used to study the conormal Sobolev continuity of the related conormal operators.

To be definite, for every nonnegative integer number $s$, such that $s+l+r-m$ is also a nonnegative integer, Proposition 3.9 and Lemma 4.5 imply that the conormal operator $\mathrm{Op}_{\sharp}^{\gamma}\left(a_{\delta}\right) \mathrm{Op}_{\sharp}^{\gamma}(b) \widetilde{\lambda}_{\delta}^{-m+1, \gamma}(Z)$ extends as a linear bounded mapping from $H_{\text {tan, } \gamma}^{s+l+r-m}(Q)$ into $H_{\text {tan, },}^{s}(Q)$; moreover, its operator norm is uniformly bounded with respect to $\gamma$ and $\delta$.

If in addition $s+l+r-m \geq 1$ and $a_{\delta}$ are scalar-valued, then $\left[\mathrm{Op}_{\sharp}^{\gamma}\left(a_{\delta}\right), \mathrm{Op}_{\sharp}^{\gamma}(b)\right] \widetilde{\lambda}_{\delta}^{-m+1, \gamma}(Z)$ extends as a linear bounded operator from $H_{\text {tan }, \gamma}^{s+l+r-m-1}(Q)$ into $H_{t a n, \gamma}^{s}(Q)$, and again its operator norm is uniformly bounded with respect to $\gamma$ and $\delta$.

These mapping properties will be usefully applied in the next Sections 4.2, 4.4.

\subsection{The interior equation}

We follow the strategy already explained in the introduction, where now the role of the operator $\lambda_{\delta}^{m-1, \gamma}(Z)$ is replaced by $\lambda_{\chi, \delta}^{m-1, \gamma}(Z)$. Since $\lambda_{\chi, \delta}^{m-1, \gamma} \in \Gamma^{m-1}$ (because of Lemma 4.2) and $u \in H_{t a n, \gamma}^{m-1}(Q)$ (from the inductive hypothesis), after Proposition 3.9 we know that $\lambda_{\chi, \delta}^{m-1, \gamma}(Z) u \in L^{2}(Q)$.

Applying $\lambda_{\chi, \delta}^{m-1, \gamma}(Z)$ to (4.5) we find that $\lambda_{\chi, \delta}^{m-1, \gamma}(Z) u$ must solve

$$
(\gamma+L)\left(\lambda_{\chi, \delta}^{\gamma, m-1}(Z) u\right)+\left[\lambda_{\chi, \delta}^{m-1, \gamma}(Z), L\right] u=\lambda_{\chi, \delta}^{m-1, \gamma}(Z) F, \quad \text { in } Q .
$$

We are going now to show that the commutator term $\left[\lambda_{\chi, \delta}^{m-1, \gamma}(Z), L\right] u$ in the above equation can be actually considered as a lower order term with respect to $\lambda_{\chi, \delta}^{m-1, \gamma}(Z) u$.

To this end, we may decompose this term as the sum of two contributions corresponding respectively to the tangential and normal components of $L$.

Firstly, in view of (1.5), (1.6), we may write the normal coefficient $A_{1}$ as

$$
A_{1}=A_{1}^{1}+A_{1}^{2}, \quad A_{1}^{1}:=\left(\begin{array}{cc}
A_{1}^{I, I} & 0 \\
0 & 0
\end{array}\right), A_{1 \mid x_{1}=0}^{2}=0,
$$

hence

$$
A_{1}^{2} \partial_{1}=H_{1} Z_{1}
$$

where $H_{1}(x)=x_{1}^{-1} A_{1}^{2}(x) \in C_{(0)}^{\infty}(Q)$. Therefore, we split $L$ as

$$
L=A_{1}^{1} \partial_{1}+L_{t a n}, \quad L_{t a n}:=H_{1} Z_{1}+\sum_{j=2}^{n+1} A_{j} Z_{j}+B .
$$

According to this, we have:

$$
\left[\lambda_{\chi, \delta}^{m-1, \gamma}(Z), L\right] u=\left[\lambda_{\chi, \delta}^{m-1, \gamma}(Z), A_{1}^{1} \partial_{1}\right] u+\left[\lambda_{\chi, \delta}^{m-1, \gamma}(Z), L_{t a n}\right] u .
$$

Note that $L_{\text {tan }}$ is just a conormal operator of order 1 , according to the terminology introduced in Section 3.2. 


\subsubsection{The tangential commutator}

Firstly, we study the tangential commutator $\left[\lambda_{\chi, \delta}^{m-1, \gamma}(Z), L_{t a n}\right] u$. Using the identity $\widetilde{\lambda}_{\delta}^{-m+1, \gamma}(Z) \lambda_{\delta}^{m-1, \gamma}(Z)=I$ and the decomposition (4.13), this tangential commutator can be written in terms of $\lambda_{\chi, \delta}^{\gamma, m-1}(Z) u$, modulo some "infinitely smooth" reminder. Indeed we compute:

$$
\begin{aligned}
& {\left[\lambda_{\chi, \delta}^{m-1, \gamma}(Z), L_{\text {tan }}\right] u=\left[\lambda_{\chi, \delta}^{m-1, \gamma}(Z), L_{\text {tan }}\right] \widetilde{\lambda}_{\delta}^{-m+1, \gamma}(Z)\left(\lambda_{\chi, \delta}^{m-1, \gamma}(Z) u+r_{m, \delta}(Z, \gamma) u\right)} \\
& =\left[\lambda_{\chi, \delta}^{m-1, \gamma}(Z), L_{\text {tan }}\right] \widetilde{\lambda}_{\delta}^{-m+1, \gamma}(Z) \lambda_{\chi, \delta}^{m-1, \gamma}(Z) u+s_{m, \delta}(x, Z, \gamma) u
\end{aligned}
$$

where we have set for short

$$
s_{m, \delta}(x, Z, \gamma):=\left[\lambda_{\chi, \delta}^{m-1, \gamma}(Z), L_{t a n}\right] \widetilde{\lambda}_{\delta}^{-m+1, \gamma}(Z) r_{m, \delta}(Z, \gamma) .
$$

\subsubsection{The normal commutator}

We notice that, due to the structure of the matrix $A_{1}^{1}$, the commutator $\left[\lambda_{\chi, \delta}^{\gamma, m-1}(Z), A_{1}^{1} \partial_{1}\right]$ acts non trivially only on the noncharacteristic component of the vector function $u$; namely we have:

$$
\left[\lambda_{\chi, \delta}^{m-1, \gamma}(Z), A_{1}^{1} \partial_{1}\right] u=\left(\begin{array}{c}
{\left[\lambda_{\chi, \delta}^{m-1, \gamma}(Z), A_{1}^{I, I} \partial_{1}\right] u^{I}} \\
0
\end{array}\right) .
$$

Therefore, we focus on the study of the first nontrivial component of the commutator term. Note that the commutator $\left[\lambda_{\chi, \delta}^{m-1, \gamma}(Z), A_{1}^{1} \partial_{1}\right]$ cannot be merely treated by the operator algebra, because of the normal derivative. This subsection is devoted to the study of the normal commutator

$$
\left[\lambda_{\chi, \delta}^{m-1, \gamma}(Z), A_{1}^{I, I} \partial_{1}\right] u^{I} .
$$

The following result is of fundamental importance.

Proposition 4.8. For all $\delta \in] 0,1], \gamma \geq 1$ and $m \in \mathbb{N}$, there exists a symbol $q_{m, \delta}(x, \xi, \gamma) \in \Gamma^{m-2}$ such that

$$
\left[\lambda_{\chi, \delta}^{m-1, \gamma}(Z), A_{1}^{I, I} \partial_{1}\right] w=q_{m, \delta}(x, Z, \gamma)\left(\partial_{1} w\right), \quad \forall w \in C_{(0)}^{\infty}(Q) .
$$

Moreover, the symbol $q_{m, \delta}(x, \xi, \gamma)$ obeys the following estimates. For all $\alpha, \beta \in \mathbb{N}^{n+1}$ there exists a positive constant $C_{m, \alpha, \beta}$, independent of $\gamma$ and $\delta$, such that

$$
\left|\partial_{\xi}^{\alpha} \partial_{x}^{\beta} q_{m, \delta}(x, \xi, \gamma)\right| \leq C_{m, \alpha, \beta} \lambda_{\delta}^{m-2-|\alpha|, \gamma}(\xi), \quad \forall x, \xi \in \mathbb{R}^{n+1} .
$$

Proof. That $q_{m, \delta}(x, \xi, \gamma)$, satisfying estimates (4.22), is a symbol in $\Gamma^{m-2}$ actually follows arguing from (4.22) and inequalities (3.14) as we already did for $\lambda_{\delta}^{m-1, \gamma}(\xi)$ and $\widetilde{\lambda}_{\delta}^{-m+1, \gamma}(\xi)$ (see Section 3.2).

For given $w \in C_{(0)}^{\infty}(Q)$, let us explicitly compute $\left(\left[\lambda_{\chi, \delta}^{m-1, \gamma}(Z), A_{1}^{I, I} \partial_{1}\right] w\right)^{\sharp}$; using 
the identity $\left(\partial_{1} w\right)^{\sharp}=e^{-x_{1}}\left(Z_{1} w\right)^{\sharp}$ and that $\lambda_{\chi, \delta}^{m-1, \gamma}(Z)$ and $Z_{1}$ commute, we find for every $x \in \mathbb{R}^{n+1}$ :

$$
\begin{aligned}
& \left(\left[\lambda_{\chi, \delta}^{m-1, \gamma}(Z), A_{1}^{I, I} \partial_{1}\right] w\right)^{\sharp}(x)=\lambda_{\chi, \delta}^{m-1, \gamma}(D)\left(A_{1}^{I, I, \natural} e^{-(\cdot)_{1}}\left(Z_{1} w\right)^{\sharp}\right)(x) \\
& -A_{1}^{I, I, \natural}(x) e^{-x_{1}}\left(Z_{1} \lambda_{\chi, \delta}^{m-1, \gamma}(Z) w\right)^{\sharp}(x) \\
& =\lambda_{\chi, \delta}^{m-1, \gamma}(D)\left(A_{1}^{I, I, !} e^{-(\cdot)_{1}}\left(Z_{1} w\right)^{\sharp}\right)(x)-A_{1}^{I, I, \downarrow}(x) e^{-x_{1}}\left(\lambda_{\chi, \delta}^{m-1, \gamma}(Z) Z_{1} w\right)^{\sharp}(x) \\
& =\lambda_{\chi, \delta}^{m-1, \gamma}(D)\left(A_{1}^{I, I, \natural} e^{-(\cdot)_{1}}\left(Z_{1} w\right)^{\sharp}\right)(x)-A_{1}^{I, I, \natural}(x) e^{-x_{1}} \lambda_{\chi, \delta}^{m-1, \gamma}(D)\left(Z_{1} w\right)^{\sharp}(x) .
\end{aligned}
$$

Observing that $\lambda_{\chi, \delta}^{m-1, \gamma}(D)$ acts on the space $\mathcal{S}\left(\mathbb{R}^{n+1}\right)$ as the convolution by the inverse Fourier transform of $\lambda_{\chi, \delta}^{m-1, \gamma}$, the preceding expression can be equivalently restated as follows:

$$
\begin{aligned}
& \left(\left[\lambda_{\chi, \delta}^{m-1, \gamma}(Z), A_{1}^{I, I} \partial_{1}\right] w\right)^{\sharp}(x) \\
& =\mathcal{F}^{-1}\left(\lambda_{\chi, \delta}^{m-1, \gamma}\right) * A_{1}^{I, I, \downarrow} e^{-(\cdot)_{1}}\left(Z_{1} w\right)^{\sharp}(x) \\
& -A_{1}^{I, I, \natural}(x) e^{-x_{1}} \mathcal{F}^{-1}\left(\lambda_{\chi, \delta}^{m-1, \gamma}\right) *\left(Z_{1} w\right)^{\sharp}(x) \\
& =\left\langle\mathcal{F}^{-1}\left(\lambda_{\chi, \delta}^{m-1, \gamma}\right), A_{1}^{I, I, \downarrow}(x-\cdot) e^{-\left(x_{1}-(\cdot)_{1}\right)}\left(Z_{1} w\right)^{\sharp}(x-\cdot)\right\rangle \\
& -A_{1}^{I, I, \downarrow}(x) e^{-x_{1}}\left\langle\mathcal{F}^{-1}\left(\lambda_{\chi, \delta}^{m-1, \gamma}\right),\left(Z_{1} w\right)^{\sharp}(x-\cdot)\right\rangle \\
& =\left\langle\eta_{\delta}^{m-1, \gamma}, \chi(\cdot) A_{1}^{I, I, \mathfrak{\natural}}(x-\cdot) e^{-\left(x_{1}-(\cdot)_{1}\right)}\left(Z_{1} w\right)^{\sharp}(x-\cdot)\right\rangle \\
& -\left\langle\eta_{\delta}^{m-1, \gamma}, \chi(\cdot) A_{1}^{I, I, \natural}(x) e^{-x_{1}}\left(Z_{1} w\right)^{\sharp}(x-\cdot)\right\rangle \\
& =\left\langle\eta_{\delta}^{m-1, \gamma}, \chi(\cdot) A_{1}^{I, I, \natural}(x-\cdot)\left(\partial_{1} w\right)^{\sharp}(x-\cdot)\right\rangle \\
& -\left\langle\eta_{\delta}^{m-1, \gamma}, \chi(\cdot) A_{1}^{I, I, \natural}(x) e^{-(\cdot)_{1}}\left(\partial_{1} w\right)^{\sharp}(x-\cdot)\right\rangle \\
& =\left\langle\eta_{\delta}^{m-1, \gamma}, \chi(\cdot)\left[A_{1}^{I, I, \natural}(x-\cdot)-A_{1}^{I, I, \downarrow)}(x) e^{-(\cdot)_{1}}\right]\left(\partial_{1} w\right)^{\sharp}(x-\cdot)\right\rangle \text {, }
\end{aligned}
$$

where $\eta_{\delta}^{m-1, \gamma}:=\mathcal{F}^{-1}\left(\lambda_{\delta}^{m-1, \gamma}\right)$, and the identity $\mathcal{F}^{-1}\left(\lambda_{\chi, \delta}^{m-1, \gamma}\right)=\chi \eta_{\delta}^{m-1, \gamma}$ (following at once from (4.9)) has been used. Just for brevity, let us further set

$$
\mathcal{K}(x, y):=\left[A_{1}^{I, I, \downarrow}(x-y)-A_{1}^{I, I, \natural}(x) e^{-y_{1}}\right] \chi(y) .
$$

Thus the identity above reads as

$$
\left(\left[\lambda_{\chi, \delta}^{m-1, \gamma}(Z), A_{1}^{I, I} \partial_{1}\right] w\right)^{\sharp}(x)=\left\langle\eta_{\delta}^{m-1, \gamma}, \mathcal{K}(x, \cdot)\left(\partial_{1} w\right)^{\sharp}(x-\cdot)\right\rangle,
$$

where the kernel $\mathcal{K}(x, y)$ is a bounded function in $C^{\infty}\left(\mathbb{R}^{n+1} \times \mathbb{R}^{n+1}\right)$, with bounded derivatives of all orders. This regularity of $\mathcal{K}$ is due to the presence of the function $\chi$ in formula (4.25); actually the vanishing of $\chi$ at infinity prevents the blow-up of the exponential factor $e^{-y_{1}}$, as $y_{1} \rightarrow-\infty$. We point out that this is just the step of our analysis of the normal commutator, where this function $\chi$ is needed.

After (4.25), we also have that $\mathcal{K}(x, 0)=0$; then, by a Taylor expansion with respect 
to $y$, we can represent the kernel $\mathcal{K}(x, y)$ as follows

$$
\mathcal{K}(x, y)=\sum_{k=1}^{n+1} b_{k}(x, y) y_{k},
$$

where $b_{k}(x, y)$ are given bounded functions in $C^{\infty}\left(\mathbb{R}^{n+1} \times \mathbb{R}^{n+1}\right)$, with bounded derivatives; it comes from (4.25) that functions $b_{k}$ can be defined so that for some $\varepsilon>1$ and all $x \in \mathbb{R}^{n+1}$ there holds

$$
\operatorname{supp} b_{k}(x, \cdot) \subseteq\{|y| \leq \varepsilon\} .
$$

Inserting (4.27) in (4.26) and using standard properties of the Fourier transform we get

$$
\begin{aligned}
& \left(\left[\lambda_{\chi, \delta}^{m-1, \gamma}(Z), A_{1}^{I, I} \partial_{1}\right] w\right)^{\sharp}(x)=\left\langle\eta_{\delta}^{m-1, \gamma}, \sum_{k=1}^{n} b_{k}(x, \cdot)(\cdot)_{k}\left(\partial_{1} w\right)^{\sharp}(x-\cdot)\right\rangle \\
& =\sum_{k=1}^{n+1}\left\langle(\cdot)_{k} \mathcal{F}^{-1}\left(\lambda_{\delta}^{m-1, \gamma}\right), b_{k}(x, \cdot)\left(\partial_{1} w\right)^{\sharp}(x-\cdot)\right\rangle \\
& =-\sum_{k=1}^{n+1}\left\langle\mathcal{F}^{-1}\left(D_{k} \lambda_{\delta}^{m-1, \gamma}\right), b_{k}(x, \cdot)\left(\partial_{1} w\right)^{\sharp}(x-\cdot)\right\rangle \\
& =-\sum_{k=1}^{n+1}\left\langle D_{k} \lambda_{\delta}^{m-1, \gamma}, \mathcal{F}^{-1}\left(b_{k}(x, \cdot)\left(\partial_{1} w\right)^{\sharp}(x-\cdot)\right)\right\rangle \\
& =-\sum_{k=1}^{n+1} \int_{\mathbb{R}^{n+1}} D_{k} \lambda_{\delta}^{m-1, \gamma}(\xi) \mathcal{F}^{-1}\left(b_{k}(x, \cdot)\left(\partial_{1} w\right)^{\sharp}(x-\cdot)\right)(\xi) d \xi \\
& =-\sum_{k=1}^{n+1}(2 \pi)^{-n-1} \int_{\mathbb{R}^{n+1}} D_{k} \lambda_{\delta}^{m-1, \gamma}(\xi)\left(\int_{\mathbb{R}^{n+1}} e^{i \xi \cdot y} b_{k}(x, y)\left(\partial_{1} w\right)^{\sharp}(x-y) d y\right) d \xi
\end{aligned}
$$

where we have set $D_{k}:=-i \partial_{\xi_{k}}$ (fo each $\left.k=1, \ldots, n+1\right)$. Note that for $w \in C_{(0)}^{\infty}(Q)$ and any $x \in \mathbb{R}^{n+1}$ the function $b_{k}(x, \cdot)\left(\partial_{1} w\right)^{\sharp}(x-\cdot)$ belongs to $\mathcal{S}\left(\mathbb{R}^{n+1}\right)$; hence the last expression in (4.29) makes sense. Henceforth, we replace $\left(\partial_{1} w\right)^{\sharp}$ by any function $v \in \mathcal{S}\left(\mathbb{R}^{n+1}\right)$. Our next goal is writing the integral operator

$$
(2 \pi)^{-n-1} \int_{\mathbb{R}^{n+1}} D_{k} \lambda_{\delta}^{m-1, \gamma}(\xi)\left(\int_{\mathbb{R}^{n+1}} e^{i \xi \cdot y} b_{k}(x, y) v(x-y) d y\right) d \xi
$$

as a pseudo-differential operator.

Firstly, we make use of the inversion formula for the Fourier transformation and Fubini's theorem to recast (4.30) as follows:

$$
\begin{aligned}
& \int_{\mathbb{R}^{n+1}} e^{i \xi \cdot y} b_{k}(x, y) v(x-y) d y \\
& =(2 \pi)^{-n-1} \int_{\mathbb{R}^{n+1}} e^{i \xi \cdot y} b_{k}(x, y)\left(\int_{\mathbb{R}^{n+1}} e^{i(x-y) \cdot \eta} \widehat{v}(\eta) d \eta\right) d y \\
& =(2 \pi)^{-n-1} \int_{\mathbb{R}^{n+1}} e^{i x \cdot \eta}\left(\int_{\mathbb{R}^{n+1}} e^{-i y \cdot(\eta-\xi)} b_{k}(x, y) d y\right) \widehat{v}(\eta) d \eta \\
& =(2 \pi)^{-n-1} \int_{\mathbb{R}^{n+1}} e^{i x \cdot \eta} \widehat{b}_{k}(x, \eta-\xi) \widehat{v}(\eta) d \eta ;
\end{aligned}
$$

for every index $k, \widehat{b}_{k}(x, \zeta)$ denotes the partial Fourier transform of $b_{k}(x, y)$ with respect to $y$. Then, inserting (4.31) into (4.30) we obtain

$$
\begin{aligned}
& (2 \pi)^{-n-1} \int_{\mathbb{R}^{n+1}} D_{k} \lambda_{\delta}^{m-1, \gamma}(\xi)\left(\int_{\mathbb{R}^{n+1}} e^{i \xi \cdot y} b_{k}(x, y) v(x-y) d y\right) d \xi \\
& =(2 \pi)^{-2 n-2} \int_{\mathbb{R}^{n+1}} D_{k} \lambda_{\delta}^{m-1, \gamma}(\xi)\left(\int_{\mathbb{R}^{n+1}} e^{i x \cdot \eta} \widehat{b}_{k}(x, \eta-\xi) \widehat{v}(\eta) d \eta\right) d \xi .
\end{aligned}
$$


Recall that for each $x \in \mathbb{R}^{n+1}$, the function $y \mapsto b_{k}(x, y)$ belongs to $C_{0}^{\infty}\left(\mathbb{R}^{n+1}\right)$ (and its compact support does not depend on $x$, see (4.28)); thus, for each $x \in \mathbb{R}^{n+1}$, $\widehat{b}_{k}(x, \zeta)$ is rapidly decreasing in $\zeta$. Because of the estimates for derivatives of $\lambda_{\delta}^{m-1, \gamma}$ and since $\widehat{v}(\eta)$ is also rapidly decreasing, Fubini's theorem can be used to change the order of the integrations within (4.32). So we get

$$
\begin{aligned}
& (2 \pi)^{-2 n-2} \int_{\mathbb{R}^{n+1}} D_{k} \lambda_{\delta}^{m-1, \gamma}(\xi)\left(\int_{\mathbb{R}^{n+1}} e^{i x \cdot \eta} \widehat{b}_{k}(x, \eta-\xi) \widehat{v}(\eta) d \eta\right) d \xi \\
& =(2 \pi)^{-2 n-2} \int_{\mathbb{R}^{n+1}} e^{i x \cdot \eta}\left(\int_{\mathbb{R}^{n+1}} \widehat{b}_{k}(x, \eta-\xi) D_{k} \lambda_{\delta}^{m-1, \gamma}(\xi) d \xi\right) \widehat{v}(\eta) d \eta \\
& =(2 \pi)^{-n-1} \int_{\mathbb{R}^{n+1}} e^{i x \cdot \eta} q_{k, m, \delta}(x, \eta, \gamma) \widehat{v}(\eta) d \eta
\end{aligned}
$$

where we have set

$$
q_{k, m, \delta}(x, \xi, \gamma):=(2 \pi)^{-n-1} \int_{\mathbb{R}^{n+1}} \widehat{b}_{k}(x, \eta) D_{k} \lambda_{\delta}^{m-1, \gamma}(\xi-\eta) d \eta .
$$

Notice that formula (4.34) defines $q_{k, m, \delta}$ as the convolution of the functions $\widehat{b}_{k}(x, \cdot)$ and $D_{k} \lambda_{\delta}^{m-1, \gamma}$; hence $q_{k, m, \delta}$ is a well defined $C^{\infty}$-function in $\mathbb{R}^{n+1} \times \mathbb{R}^{n+1}$.

The proof of Proposition 4.8 will be accomplished, once the following Lemma will be proved. The proof of Lemma 4.9 is given in [17].

Lemma 4.9. For every $m \in \mathbb{N}, k=1, \ldots, n+1$ and all $\alpha, \beta \in N^{n+1}$ there exists a positive constant $C_{k, m, \alpha, \beta}$, independent of $\gamma$ and $\delta$, such that

$$
\left|\partial_{\xi}^{\alpha} \partial_{x}^{\beta} q_{k, m, \delta}(x, \xi, \gamma)\right| \leq C_{k, m, \alpha, \beta} \lambda_{\delta}^{m-2-|\alpha|, \gamma}(\xi), \quad \forall x, \xi \in \mathbb{R}^{n+1} .
$$

It comes from Lemma 4.9 and the left inequality in (3.14) that, for each index $k$, the function $q_{k, m, \delta}$ is a symbol in $\Gamma^{m-2}$; notice however that the set $\left\{q_{k, m \delta}\right\}_{0<\delta \leq 1}$ is bounded in $\Gamma^{m-1}$ but not in $\Gamma^{m-2}$.

End of the proof of Proposition 4.8. The last row of (4.33) provides the desired representation of (4.30) as a pseudo-differential operator; actually it gives the identity

$$
(2 \pi)^{-n-1} \int_{\mathbb{R}^{n+1}} D_{k} \lambda_{\delta}^{m-1, \gamma}(\xi)\left(\int_{\mathbb{R}^{n+1}} e^{i \xi \cdot y} b_{k}(x, y) v(x-y) d y\right) d \xi=\mathrm{Op}^{\gamma}\left(q_{k, m, \delta}\right) v(x),
$$

for every $v \in \mathcal{S}\left(\mathbb{R}^{n+1}\right)$.

Inserting the above formula (with $\left.v=\left(\partial_{1} w\right)^{\sharp}\right)$ into (4.29) finally gives

$$
\left(\left[\lambda_{\chi, \delta}^{m-1, \gamma}(Z), A_{1}^{I, I} \partial_{1}\right] w\right)^{\sharp}(x)=\mathrm{Op}^{\gamma}\left(q_{m, \delta}\right)\left(\partial_{1} w\right)^{\sharp}(x),
$$

where $q_{m, \delta}=q_{m, \delta}(x, \xi, \gamma)$ is the symbol in $\Gamma^{m-2}$ defined by

$$
q_{m, \delta}(x, \xi, \gamma):=-\sum_{k=1}^{n+1} q_{k, m, \delta}(x, \xi, \gamma) .
$$

Of course, formula (4.21) is equivalent to (4.36), in view of (3.21). Estimates (4.22) are satisfied by $q_{m, \delta}$ by summation over $k$ of the similar estimate satisfied by $q_{m, \delta, k}$ (cf. Lemma 4.9).

This ends the proof of Proposition 4.8. 
Now, we are going to show how the representation in (4.21) can be exploited to treat the normal commutator as a lower order term in the equation (4.16) satisfied by $\lambda_{\chi, \delta}^{m-1, \gamma}(Z) u$.

Firstly, we notice that formula (4.21) has been deduced for smooth functions $w$, while $u$ is just an $L^{2}$-function (actually it belongs to $H_{t a n}^{m-1}(Q)$ ), by the inductive hypothesis). In order to use (4.21) for $u$, we need approximating the latter by smooth functions. This can be done by the help of [21, Prop. 1, Th. 1]; from there, we know there exists a sequence $\left\{u_{\nu}\right\}_{\nu}$ in $C_{(0)}^{\infty}(Q)$ such that

$$
\begin{aligned}
& u_{\nu} \rightarrow u, \quad \text { in } L^{2}(Q), \\
& L u_{\nu} \rightarrow L u, \quad \text { in } L^{2}(Q), \\
& u_{\nu \mid x_{1}=0}^{I} \rightarrow u_{\mid x_{1}=0}^{I}, \quad \text { in } H^{-1 / 2}(\Sigma), \quad \text { as } \nu \rightarrow+\infty .
\end{aligned}
$$

For each index $\nu$, the regular function $u_{\nu} \in C_{(0)}^{\infty}(Q)$ solves the same BVP as the function $u$, with new data $F_{\nu}, G_{\nu}$ defined by

$$
F_{\nu}:=(\gamma+L) u_{\nu} \quad G_{\nu}:=M u_{\nu \mid x_{1}=0} .
$$

It immediately follows from (4.38) that the regular data $F_{\nu}, G_{\nu}$ approximate the original data $F, G$ by

$$
F_{\nu} \rightarrow F, \quad \text { in } L^{2}(Q), \quad G_{\nu} \rightarrow G, \quad \text { in } H^{-1 / 2}(\Sigma), \quad \text { as } \nu \rightarrow+\infty .
$$

The same analysis performed to the BVP (4.5) can be applied to the BVP solved by $u_{\nu}$, for each $\nu$; in particular, we find that $\lambda_{\chi, \delta}^{m-1, \gamma}(Z) u_{\nu}$ satisfies the analogue to (4.16), where $F$ is replaced by $F_{\nu}$. Because of the regularity of $u_{\nu}$, formula (4.21) can be used to represent the normal commutator term $\left[\lambda_{\chi, \delta}^{m-1, \gamma}(Z), A_{1}^{I, I} \partial_{1}\right] u_{\nu}^{I}$. Directly from system $(\gamma+L) u_{\nu}=F_{\nu}$, we can express $\partial_{1} u_{\nu}^{I}$ as a function of tangential derivatives of $u_{\nu}$ in the form

$$
\partial_{1} u_{\nu}^{I}=\left(A_{1}^{I, I}\right)^{-1} F_{\nu}^{I}+\mathcal{L}^{I} u_{\nu},
$$

where $\mathcal{L}^{I}=\mathcal{L}^{I}(x, Z, \gamma)$ denotes the tangential partial differential operator

$$
\mathcal{L}^{I} u_{\nu}:=-\left(A_{1}^{I, I}\right)^{-1}\left[\gamma u_{\nu}^{I}+H_{2} Z_{1} u_{\nu}^{I I}+\left(\sum_{j=2}^{n+1} A_{j} Z_{j} u_{\nu}+B u_{\nu}\right)^{I}\right]
$$

and we have set $H_{2}:=x_{1}^{-1} A_{1}^{I, I I}$ (recall that $H_{2} \in C_{(0)}^{\infty}(Q)$ since $A_{1 \mid x_{1}=0}^{I, I I}=0$ ). Inserting (4.41) into (4.21), written for $w=u_{\nu}^{I}$, leads to

$$
\left[\lambda_{\chi, \delta}^{m-1, \gamma}(Z), A_{1}^{I, I} \partial_{1}\right] u_{\nu}^{I}=q_{m, \delta}(x, Z, \gamma)\left(\left(A_{1}^{I, I}\right)^{-1} F_{\nu}^{I}+\mathcal{L}^{I} u_{\nu}\right) .
$$

On the other hand, plugging

$$
\tilde{\lambda}_{\delta}^{-m+1, \gamma}(Z) \lambda_{\chi, \delta}^{m-1, \gamma}(Z)+\widetilde{\lambda}_{\delta}^{-m+1, \gamma}(Z) r_{m, \delta}(Z, \gamma)=I
$$

into (4.42) gives

$$
\begin{aligned}
& {\left[\lambda_{\chi, \delta}^{m-1, \gamma}(Z), A_{1}^{I, I} \partial_{1}\right] u_{\nu}^{I}=q_{m, \delta}(x, Z, \gamma)\left(\left(A_{1}^{I, I}\right)^{-1} F_{\nu}^{I}\right)} \\
& +q_{m, \delta}(x, Z, \gamma) \mathcal{L}^{I} \widetilde{\lambda}_{\delta}^{-m+1, \gamma}(Z)\left(\lambda_{\chi, \delta}^{m-1, \gamma}(Z) u_{\nu}\right) \\
& +q_{m, \delta}(x, Z, \gamma) \mathcal{L}^{I} \widetilde{\lambda}_{\delta}^{-m+1, \gamma}(Z) r_{m, \delta}(Z, \gamma) u_{\nu} .
\end{aligned}
$$


Now, we let $\nu \rightarrow+\infty$.

From equation (4.5) (written for $u$ and $u_{\nu}$ ), and using that $u_{\nu}^{\sharp} \rightarrow u^{\sharp}$ in $L^{2}\left(\mathbb{R}^{n+1}\right)$, one finds

$$
\begin{aligned}
& \left(A_{1} \partial_{1} u_{\nu}\right)^{\sharp}=F_{\nu}^{\sharp}-\gamma u_{\nu}^{\sharp}-\sum_{j=2}^{n+1} A_{j}^{\natural} \partial_{j}\left(u_{\nu}^{\sharp}\right)-B^{\natural} u_{\nu}^{\sharp} \\
& \rightarrow F^{\sharp}-\gamma u^{\sharp}-\sum_{j=2}^{n+1} A_{j}^{\natural} \partial_{j}\left(u^{\sharp}\right)-B^{\natural} u^{\sharp}=\left(A_{1} \partial_{1} u\right)^{\sharp}, \quad \text { in } \mathcal{S}^{\prime}\left(\mathbb{R}^{n+1}\right) .
\end{aligned}
$$

Then from

$$
\lambda_{\chi, \delta}^{m-1, \gamma}(D)\left(\left(A_{1}^{I, I} \partial_{1} u_{\nu}^{I}\right)^{\sharp}\right) \rightarrow \lambda_{\chi, \delta}^{m-1, \gamma}(D)\left(\left(A_{1}^{I, I} \partial_{1} u^{I}\right)^{\sharp}\right), \text { in } \mathcal{S}^{\prime}\left(\mathbb{R}^{n+1}\right)
$$

and the continuity of $\sharp^{-1}$ as a linear operator from $\mathcal{S}^{\prime}\left(\mathbb{R}^{n+1}\right) \subset \mathcal{D}^{\prime}\left(\mathbb{R}^{n+1}\right)$ to $\mathcal{D}^{\prime}(Q)$, we get

$$
\lambda_{\chi, \delta}^{m-1, \gamma}(Z)\left(A_{1}^{I, I} \partial_{1} u_{\nu}^{I}\right) \rightarrow \lambda_{\chi, \delta}^{m-1, \gamma}(Z)\left(A_{1}^{I, I} \partial_{1} u^{I}\right), \text { in } \mathcal{D}^{\prime}(Q) .
$$

On the other hand, again from $u_{\nu}^{\sharp} \rightarrow u^{\sharp}$ in $L^{2}\left(\mathbb{R}^{n+1}\right)$ we find

$$
\lambda_{\chi, \delta}^{m-1, \gamma}(Z)\left(u_{\nu}\right) \rightarrow \lambda_{\chi, \delta}^{m-1, \gamma}(Z)(u), \quad \text { in } \mathcal{D}^{\prime}(Q),
$$

hence

$$
A_{1}^{I, I} \partial_{1}\left(\lambda_{\chi, \delta}^{m-1, \gamma}(Z)\left(u_{\nu}^{I}\right)\right) \rightarrow A_{1}^{I, I} \partial_{1}\left(\lambda_{\chi, \delta}^{m-1, \gamma}(Z)\left(u^{I}\right)\right), \quad \text { in } \mathcal{D}^{\prime}(Q) .
$$

Adding (4.44), (4.46) then gives

$$
\left[\lambda_{\chi, \delta}^{m-1, \gamma}(Z), A_{1}^{I, I} \partial_{1}\right] u_{\nu}^{I} \rightarrow\left[\lambda_{\chi, \delta}^{m-1, \gamma}(Z), A_{1}^{I, I} \partial_{1}\right] u^{I}, \quad \text { in } \mathcal{D}^{\prime}(Q) .
$$

As to the right-hand side of (4.43), all the operators, acting on $F_{\nu}$ and $u_{\nu}$, that appear are conormal operators. Hence the $L^{2}$-convergences $u_{\nu} \rightarrow u$ and $F_{\nu} \rightarrow F$ and the fact that conormal operators continuously extend to the space of distributions $u \in \mathcal{D}^{\prime}(Q)$, for which $u^{\sharp} \in \mathcal{S}^{\prime}\left(\mathbb{R}^{n+1}\right)$, give the convergences

$$
\begin{aligned}
& q_{m, \delta}(x, Z, \gamma)\left(\left(A_{1}^{I, I}\right)^{-1} F_{\nu}^{I}\right) \rightarrow q_{m, \delta}(x, Z, \gamma)\left(\left(A_{1}^{I, I}\right)^{-1} F^{I}\right), \\
& q_{m, \delta}(x, Z, \gamma) \mathcal{L}^{I} \widetilde{\lambda}_{\delta}^{-m+1, \gamma}(Z)\left(\lambda_{\chi, \delta}^{m-1, \gamma}(Z) u_{\nu}\right) \\
& \rightarrow q_{m, \delta}(x, Z, \gamma) \mathcal{L}^{I} \widetilde{\lambda}_{\delta}^{-m+1, \gamma}(Z)\left(\lambda_{\chi, \delta}^{m-1, \gamma}(Z) u\right), \\
& q_{m, \delta}(x, Z, \gamma) \mathcal{L}^{I} \widetilde{\lambda}_{\delta}^{-m+1, \gamma}(Z) r_{m, \delta}(Z, \gamma) u_{\nu} \\
& \rightarrow q_{m, \delta}(x, Z, \gamma) \mathcal{L}^{I} \widetilde{\lambda}_{\delta}^{-m+1, \gamma}(Z) r_{m, \delta}(Z, \gamma) u, \quad \text { in } \mathcal{D}^{\prime}(Q) .
\end{aligned}
$$

Therefore, the uniqueness of the limit in $\mathcal{D}^{\prime}(Q)$ together with (4.47), (4.48) imply that (4.43) holds true for the $L^{2}$-solution $u$ of (4.5), that is

$$
\begin{aligned}
& {\left[\lambda_{\chi, \delta}^{m-1, \gamma}(Z), A_{1}^{I, I} \partial_{1}\right] u^{I}=q_{m, \delta}(x, Z, \gamma)\left(\left(A_{1}^{I, I}\right)^{-1} F^{I}\right)} \\
& +q_{m, \delta}(x, Z, \gamma) \mathcal{L}^{I} \widetilde{\lambda}_{\delta}^{-m+1, \gamma}(Z)\left(\lambda_{\chi, \delta}^{m-1, \gamma}(Z) u\right) \\
& +q_{m, \delta}(x, Z, \gamma) \mathcal{L}^{I} \widetilde{\lambda}_{\delta}^{-m+1, \gamma}(Z) r_{m, \delta}(Z, \gamma) u
\end{aligned}
$$

Let us come back to the commutator term $\left[\lambda_{\chi, \delta}^{m-1, \gamma}(Z), L\right] u$ appearing in the interior equation (4.16). 
Substituting (4.19), (4.49) into (4.18) gives for this term the following representation

$$
\left[\lambda_{\chi, \delta}^{m-1, \gamma}(Z), L\right] u=\rho_{m, \delta}(x, Z, \gamma)\left(\lambda_{\chi, \delta}^{m-1, \gamma}(Z) u\right)+\tau_{m, \delta}(x, Z, \gamma) u+\eta_{m, \delta}(x, Z, \gamma) F,
$$

where we have set for short:

$$
\begin{aligned}
\rho_{m, \delta}(x, Z, \gamma) & :=\left[\lambda_{\chi, \delta}^{m-1, \gamma}(Z), L_{t a n}\right] \widetilde{\lambda}_{\delta}^{-m+1, \gamma}(Z)+\left(\begin{array}{c}
q_{m, \delta}(x, Z, \gamma) \mathcal{L}^{I} \widetilde{\lambda}_{\delta}^{-m+1, \gamma}(Z) \\
0
\end{array}\right) \\
\tau_{m, \delta}(x, Z, \gamma) & :=s_{m, \delta}(x, Z, \gamma)+\left(\begin{array}{c}
q_{m, \delta}(x, Z, \gamma) \mathcal{L}^{I} \widetilde{\lambda}_{\delta}^{-m+1, \gamma}(Z) r_{m, \delta}(Z, \gamma) \\
0
\end{array}\right) \\
\eta_{m, \delta}(x, Z, \gamma) & :=\left(\begin{array}{cr}
q_{m, \delta}(x, Z, \gamma)\left(A_{1}^{I, I}\right)^{-1} & 0 \\
0 & 0
\end{array}\right) .
\end{aligned}
$$

Consequently, the interior equation (4.16) can be restated as

$$
\begin{aligned}
& \left(\gamma+L+\rho_{m, \delta}(x, Z, \gamma)\right)\left(\lambda_{\chi, \delta}^{m-1, \gamma}(Z) u\right)+\tau_{m, \delta}(x, Z, \gamma) u+\eta_{m, \delta}(x, Z, \gamma) F \\
& =\lambda_{\chi, \delta}^{m-1, \gamma}(Z) F, \quad \text { in } Q .
\end{aligned}
$$

Since $L_{\text {tan }}$ and $\mathcal{L}^{I}$ are conormal operators with symbol in $\Gamma^{1}$, Lemma 4.5 and Proposition 4.8 imply that $\rho_{m, \delta}(x, Z, \gamma)$ is a conormal operator with symbol in $\Gamma^{0}$, for each $0<\delta \leq 1$; moreover the family of symbols $\left\{\rho_{m, \delta}\right\}_{0<\delta \leq 1}$ is a bounded subset of $\Gamma^{0}$.

$\rho_{m, \delta}(x, Z, \gamma)$ can be regarded as a lower order term in the equation (4.52) of type $B_{2}$, see assumption (4).

Concerning the terms $\tau_{m, \delta}(x, Z, \gamma) u, \eta_{m, \delta}(x, Z, \gamma) F$, they can be both moved into the right-hand side of (4.52), to be treated as a part of the interior source term, as will be detailed in Section 4.4 .

\subsection{The boundary condition}

Now we are going to look for an appropriate boundary condition to be coupled with the interior equation (4.16), in order to state a BVP solved by $\lambda_{\chi, \delta}^{m-1, \gamma}(Z) u$.

To this end, it is worth-while to make an additional hypothesis about the smooth function $\chi$ involved in the definition of $\lambda_{\chi, \delta}^{m-1, \gamma}(Z)$. We assume that $\chi$ has the form:

$$
\forall x=\left(x_{1}, x^{\prime}\right) \in \mathbb{R}^{n+1}, \quad \chi(x)=\chi_{1}\left(x_{1}\right) \widetilde{\chi}\left(x^{\prime}\right),
$$

where $\chi_{1} \in C^{\infty}(\mathbb{R})$ and $\tilde{\chi} \in C^{\infty}(\Sigma)$ are given positive even functions, to be chosen in such a way that conditions (4.8) are satisfied.

As it was done for the analysis of the normal commutator (cf. Proposition 4.8), we start our reasoning by dealing with smooth functions. In this case, we are able to prove the following

Proposition 4.10. Assume that $\chi$ obeys the assumptions (4.8), (4.53). Then, for all $\delta \in] 0,1], \gamma \geq 1$ and $m \in \mathbb{N}$ there exists a $\gamma$-depending symbol $b_{m, \delta}^{\prime}=$ $b_{m, \delta}^{\prime}\left(\xi^{\prime}, \gamma\right) \in \Gamma^{m-1}$ in $\Sigma\left(x^{\prime}=\left(x_{2}, \ldots, x_{n+1}\right)\right.$ are the space variables in $\Sigma$ and $\xi^{\prime}:=$ 
$\left(\xi_{2}, \ldots, \xi_{n+1}\right)$ the Fourier dual variables) such that for all functions $w \in C_{(0)}^{\infty}(Q)$ there holds

$$
\forall x^{\prime} \in \Sigma, \quad\left(\lambda_{\chi, \delta}^{m-1, \gamma}(Z) w\right)_{\mid x_{1}=0}\left(x^{\prime}\right)=b_{m, \delta}^{\prime}\left(D^{\prime}, \gamma\right)\left(w_{\mid x_{1}=0}\right)\left(x^{\prime}\right),
$$

where we denote $D^{\prime}=\left(D_{2}, \ldots, D_{n+1}\right)$ and $D_{j}=-i \partial_{j}$ for $j=2, \ldots, n+1$, and $b_{m, \delta}^{\prime}\left(D^{\prime}, \gamma\right)$ stands for the ordinary pseudo-differential operator with symbol $b_{m, \delta}^{\prime}$ in $\Sigma$.

Proof. Let $w \in C_{(0)}^{\infty}(Q)$; to find a symbol $b_{m, \delta}^{\prime}$ satisfying (4.54), from (4.9) we firstly compute

$$
\begin{aligned}
& \left(\lambda_{\chi, \delta}^{m-1, \gamma}(Z) w\right)^{\sharp}(x)=\lambda_{\chi, \delta}^{m-1, \gamma}(D) w^{\sharp}(x) \\
& =\left(\mathcal{F}^{-1}\left(\lambda_{\chi, \delta}^{m-1, \gamma}\right) * w^{\sharp}\right)(x)=\left\langle\mathcal{F}^{-1}\left(\lambda_{\chi, \delta}^{m-1, \gamma}\right), w^{\sharp}(x-\cdot)\right\rangle \\
& =\left\langle\mathcal{F}^{-1}\left(\lambda_{\delta}^{m-1, \gamma}\right), \chi(\cdot) e^{\frac{\left.x_{1}-(\cdot)\right)_{1}}{2}} w\left(e^{x_{1}-(\cdot)_{1}}, x^{\prime}-(\cdot)^{\prime}\right)\right\rangle, \quad \forall\left(x_{1}, x^{\prime}\right) \in \mathbb{R}^{n+1},
\end{aligned}
$$

hence $\forall x_{1}>0, \forall x^{\prime} \in \Sigma$,

$$
\begin{aligned}
& \lambda_{\chi, \delta}^{m-1, \gamma}(Z) w(x)=\left\langle\mathcal{F}^{-1}\left(\lambda_{\delta}^{m-1, \gamma}\right), \chi(\cdot) e^{\frac{-(\cdot)_{1}}{2}} w\left(x_{1} e^{-(\cdot)_{1}}, x^{\prime}-(\cdot)^{\prime}\right)\right\rangle \\
& =\left\langle\lambda_{\delta}^{m-1, \gamma}, \mathcal{F}^{-1}\left(\chi(\cdot) e^{\frac{-(\cdot)_{1}}{2}} w\left(x_{1} e^{-(\cdot)_{1}}, x^{\prime}-(\cdot)^{\prime}\right)\right)\right\rangle \\
& =(2 \pi)^{-n} \int \lambda_{\delta}^{m-1, \gamma}(\xi)\left(\int e^{i \xi \cdot y} \chi(y) e^{-\frac{y_{1}}{2}} w\left(x_{1} e^{-y_{1}}, x^{\prime}-y^{\prime}\right) d y\right) d \xi .
\end{aligned}
$$

The presence of $\chi$ and the regularity of $w$ legitimate all the above calculations. Setting $x_{1}=0$ in the last expression above, we deduce the corresponding expression for the trace on the boundary of $\lambda_{\chi, \delta}^{m-1, \gamma}(Z) w$

$$
\begin{aligned}
& \left(\lambda_{\chi, \delta}^{m-1, \gamma}(Z) w\right)_{\mid x_{1}=0}\left(x^{\prime}\right) \\
& =(2 \pi)^{-n-1} \int \lambda_{\delta}^{m-1, \gamma}(\xi)\left(\int e^{i \xi \cdot y} \chi(y) e^{-\frac{y_{1}}{2}}\left(w_{\mid x_{1}=0}\right)\left(x^{\prime}-y^{\prime}\right) d y\right) d \xi .
\end{aligned}
$$

Now we substitute (4.53) into the $y$-integral appearing in the last expression above; then Fubini's theorem gives

$$
\begin{aligned}
& \int e^{i \xi \cdot y} \chi_{1}\left(y_{1}\right) \widetilde{\chi}\left(y^{\prime}\right) e^{-\frac{y_{1}}{2}}\left(w_{\mid x_{1}=0}\right)\left(x^{\prime}-y^{\prime}\right) d y \\
& =\int e^{i \xi^{\prime} \cdot y^{\prime}}\left(\int e^{i \xi_{1} y_{1}} e^{-\frac{y_{1}}{2}} \chi_{1}\left(y_{1}\right) d y_{1}\right) \tilde{\chi}\left(y^{\prime}\right)\left(w_{\mid x_{1}=0}\right)\left(x^{\prime}-y^{\prime}\right) d y^{\prime} \\
& =\left(e^{\frac{(\cdot)_{1}}{2}} \chi_{1}\right)^{\wedge_{1}}\left(\xi_{1}\right) \int e^{i \xi^{\prime} \cdot y^{\prime}} \widetilde{\chi}\left(y^{\prime}\right)\left(w_{\mid x_{1}=0}\right)\left(x^{\prime}-y^{\prime}\right) d y^{\prime},
\end{aligned}
$$

where $\wedge_{1}$ is used to denote the one-dimensional Fourier transformation with respect to $y_{1}$. Writing, by the inversion formula, $\left(w_{\mid x_{1}=0}\right)\left(x^{\prime}-y^{\prime}\right)=$ $(2 \pi)^{-n} \int e^{i\left(x^{\prime}-y^{\prime}\right) \cdot \eta^{\prime}} \widehat{w_{\mid x_{1}=0}}\left(\eta^{\prime}\right) d \eta^{\prime}$ and using once more Fubini's theorem, we further obtain

$$
\begin{aligned}
& \int e^{i \xi^{\prime} \cdot y^{\prime}} \widetilde{\chi}\left(y^{\prime}\right)\left(w_{\mid x_{1}=0}\right)\left(x^{\prime}-y^{\prime}\right) d y^{\prime} \\
& =(2 \pi)^{-n} \int e^{i \xi^{\prime} \cdot y^{\prime}} \widetilde{\chi}\left(y^{\prime}\right)\left(\int e^{i\left(x^{\prime}-y^{\prime}\right) \cdot \eta^{\prime}} \widehat{w_{\mid x_{1}=0}}\left(\eta^{\prime}\right) d \eta^{\prime}\right) d y^{\prime} \\
& =\int e^{i x^{\prime} \cdot \eta^{\prime}}\left((2 \pi)^{-n} \int e^{i\left(\xi^{\prime}-\eta^{\prime}\right) \cdot y^{\prime}} \widetilde{\chi}\left(y^{\prime}\right) d y^{\prime}\right) \widehat{w_{\mid x_{1}=0}}\left(\eta^{\prime}\right) d \eta^{\prime} \\
& =(2 \pi)^{-n} \int e^{i x^{\prime} \cdot \eta^{\prime}} \widehat{\widetilde{\chi}}\left(\xi^{\prime}-\eta^{\prime}\right) \widehat{w_{\mid x_{1}=0}}\left(\eta^{\prime}\right) d \eta^{\prime} ;
\end{aligned}
$$


here $\wedge$ is used here to denote the $n$-dimensional Fourier transformation with respect to $x^{\prime}$. Inserting (4.56), (4.57) into (4.55) then leads to

$$
\begin{aligned}
& \left(\lambda_{\chi, \delta}^{m-1, \gamma}(Z) w\right)_{\mid x_{1}=0}\left(x^{\prime}\right)= \\
& (2 \pi)^{-n-1} \int \lambda_{\delta}^{m-1, \gamma}(\xi)\left(e^{\frac{(\cdot)_{1}}{2}} \chi_{1}\right)^{\wedge 1}\left(\xi_{1}\right)\left((2 \pi)^{-n} \int e^{i x^{\prime} \cdot \eta^{\prime}} \widehat{\widetilde{\chi}}\left(\xi^{\prime}-\eta^{\prime}\right) \widehat{w_{\mid x_{1}=0}}\left(\eta^{\prime}\right) d \eta^{\prime}\right) d \xi .
\end{aligned}
$$

Because $\left(e^{\frac{(\cdot)_{1}}{2}} \chi_{1}\right)^{\wedge_{1}} \in \mathcal{S}(\mathbb{R}), \widehat{\tilde{\chi}} \in \mathcal{S}(\Sigma)$ and $\widehat{w_{\mid x_{1}=0}} \in \mathcal{S}(\Sigma)$, the double integral

$$
\iint e^{i x^{\prime} \cdot \eta^{\prime}} \lambda_{\delta}^{m-1, \gamma}(\xi)\left(e^{\frac{(\cdot)_{1}}{2}} \chi_{1}\right)^{\wedge_{1}}\left(\xi_{1}\right) \widehat{\widetilde{\chi}}\left(\xi^{\prime}-\eta^{\prime}\right) \widehat{w_{\mid x_{1}=0}}\left(\eta^{\prime}\right) d \eta^{\prime} d \xi
$$

converges absolutely; hence Fubini's theorem can be used to find

$$
\left(\lambda_{\chi, \delta}^{m-1, \gamma}(Z) w\right)_{\mid x_{1}=0}\left(x^{\prime}\right)=(2 \pi)^{-n} \int e^{i x^{\prime} \cdot \eta^{\prime}} b_{m, \delta}^{\prime}\left(\eta^{\prime}, \gamma\right) \widehat{w_{\mid x_{1}=0}}\left(\eta^{\prime}\right) d \eta^{\prime},
$$

where we have set

$$
b_{m, \delta}^{\prime}\left(\eta^{\prime}, \gamma\right):=(2 \pi)^{-n-1} \int \lambda_{\delta}^{m-1, \gamma}(\xi)\left(e^{\frac{(\cdot)_{1}}{2}} \chi_{1}\right)^{\wedge_{1}}\left(\xi_{1}\right) \widehat{\tilde{\chi}}\left(\xi^{\prime}-\eta^{\prime}\right) d \xi .
$$

This shows the identity (4.54). It remains now to prove that $b_{m, \delta}^{\prime}$ defined by (4.61) is a $\gamma$-symbol in $\Gamma^{m-1}$. This will be accomplished, once the following Lemma will be proved.

Lemma 4.11. Let $b_{m, \delta}^{\prime}$ be defined by (4.61). Then the following estimates are satisfied: for all $\alpha^{\prime}=\left(\alpha_{2}, \ldots, \alpha_{n+1}\right) \in \mathbb{N}^{n}$ there exists a positive constant $C_{m, \alpha^{\prime}}$, independent of $\gamma$ and $\delta$, such that

$$
\left|\partial_{\xi^{\prime}}^{\alpha^{\prime}} b_{m, \delta}^{\prime}\left(\xi^{\prime}, \gamma\right)\right| \leq C_{m, \alpha^{\prime}} \lambda_{\delta}^{m-1-\left|\alpha^{\prime}\right|, \gamma}\left(\xi^{\prime}\right), \quad \forall \xi^{\prime} \in \mathbb{R}^{n} .
$$

The proof of Lemma 4.11 is similar to that for Lemma 4.9.

Let us now illustrate how formula (4.54) can be used to derive the desired boundary condition satisfied by $\lambda_{\chi, \delta}^{m-1, \gamma}(Z) u$.

Again, let $u$ be the $L^{2}$-solution to the BVP (4.5) and $\left\{u_{\nu}\right\}_{\nu=1}^{+\infty}$ the corresponding sequence in $C_{(0)}^{\infty}(Q)$, approximating $u$ in the sense of (4.38).

The last convergence in (4.38) and the Sobolev continuity of standard pseudodifferential operators (see Proposition 3.8) gives in particular that

$$
b_{m, \delta}^{\prime}\left(D^{\prime}, \gamma\right)\left(u_{\nu \mid x_{1}=0}^{I}\right) \rightarrow b_{m, \delta}^{\prime}\left(D^{\prime}, \gamma\right)\left(u_{\mid x_{1}=0}^{I}\right), \quad \text { in } H^{-m+1 / 2}(\Sigma) .
$$

On the other hand, (4.38) and (4.52) (written both for $u$ and $u_{\nu}$ ) can be used to prove that the traces $\left(\lambda_{\chi, \delta}^{m-1, \gamma}(Z) u^{I}\right)_{\mid x_{1}=0}$ and $\left(\lambda_{\chi, \delta}^{m-1, \gamma}(Z) u_{\nu}^{I}\right)_{\mid x_{1}=0}$, for each $\nu$, are well defined in $H^{-1 / 2}(\Sigma)$ and the convergence

$$
\left(\lambda_{\chi, \delta}^{m-1, \gamma}(Z) u_{\nu}^{I}\right)_{\mid x_{1}=0} \rightarrow\left(\lambda_{\chi, \delta}^{m-1, \gamma}(Z) u^{I}\right)_{\mid x_{1}=0}
$$

holds true, at least in $\mathcal{D}^{\prime}(\Sigma)$. The proof of this assertion is given in [17, Lemma 25]. Since $u_{\nu}$ are smooth functions, from Proposition 4.10 it comes that for each $\nu$ :

$$
\left(\lambda_{\chi, \delta}^{m-1, \gamma}(Z) u_{\nu}^{I}\right)_{\mid x_{1}=0}=b_{m, \delta}^{\prime}\left(D^{\prime}, \gamma\right)\left(u_{\nu \mid x_{1}=0}^{I}\right) .
$$


Then, letting $\nu \rightarrow+\infty$, (4.63) and (4.64) yield

$$
\left(\lambda_{\chi, \delta}^{m-1, \gamma}(Z) u^{I}\right)_{\mid x_{1}=0}=b_{m, \delta}^{\prime}\left(D^{\prime}, \gamma\right)\left(u_{\mid x_{1}=0}^{I}\right) .
$$

Recalling that $M=\left(I_{d}, 0\right)$, from the boundary condition in (4.5) and (4.66) we immediately find

$$
\begin{aligned}
& \left(M\left(\lambda_{\chi, \delta}^{m-1, \gamma}(Z) u\right)\right)_{\mid x_{1}=0}=M\left(\lambda_{\chi, \delta}^{m-1, \gamma}(Z) u\right)_{\mid x_{1}=0}^{I}=M b_{m, \delta}^{\prime}\left(D^{\prime}, \gamma\right)\left(u_{\mid x_{1}=0}^{I}\right) \\
& =b_{m, \delta}^{\prime}\left(D^{\prime}, \gamma\right)\left(M u_{x_{1}=0}\right)=b_{m, \delta}^{\prime}\left(D^{\prime}, \gamma\right) G .
\end{aligned}
$$

\subsection{Derivation of the conormal regularity at the order $m$}

We are now in the position to get the desired conormal regularity of the solution $u$ of (4.5), under the assumptions that $F \in H_{t a n, \gamma}^{m+1}(Q), G \in H_{\gamma}^{m+1}(\Sigma)$. As before explained, from the inductive hypothesis we already know that $u \in H_{t a n, \gamma}^{m-1}(Q)$, with $u_{\mid x_{1}=0}^{I} \in H_{\gamma}^{m-1}(\Sigma)$, provided that $\gamma \geq \gamma_{m-1}$ and $\gamma_{m-1} \geq 1$ is sufficiently large. Moreover the solution obeys the estimate (4.7) of order $m-1$. From the calculations made in the previous Sections 4.2, 4.3, we know that the function $\lambda_{\chi, \delta}^{m-1, \gamma}(Z) u \in L^{2}(Q)$ solves the following BVP

$$
\begin{aligned}
& \left(\gamma+L+\rho_{m, \delta}(x, Z, \gamma)\right)\left(\lambda_{\chi, \delta}^{\gamma, m-1}(Z) u\right) \\
& =\left(\lambda_{\chi, \delta}^{m-1, \gamma}(Z)-\eta_{m, \delta}(x, Z, \gamma)\right) F-\tau_{m, \delta}(x, Z, \gamma) u, \text { in } Q, \\
& \quad M\left(\lambda_{\chi, \delta}^{m-1, \gamma}(Z) u\right)=b_{m, \delta}^{\prime}\left(D^{\prime}, \gamma\right) G, \text { on } \Sigma .
\end{aligned}
$$

The problem for $\lambda_{\chi, \delta}^{m-1, \gamma}(Z) u$ reads as the original BVP (4.5) solved by $u$, where the role of the lower order term $B$ is played here by the conormal operator $B+$ $\rho_{m, \delta}(x, Z, \gamma)$. As already discussed in the end of Section 4.2, in view of Proposition 3.9 and Lemma 4.5 , the symbol of $B+\rho_{m, \delta}(x, Z, \gamma)$ belongs to $\Gamma^{0}$ and $\left\{\rho_{m, \delta}\right\}_{0<\delta \leq 1}$ is a bounded subset of $\Gamma^{0}$.

As regards to the terms $\tau_{m, \delta}(x, Z, \gamma) u$ and $\eta_{m, \delta}(x, Z, \gamma) F$ appearing into the righthand side of (4.68), they can be regarded as a part of the source term in the interior equation (4.68): this is the reason why we moved them to the right side of the equality.

Let us firstly focus on $\tau_{m, \delta}(x, Z, \gamma) u$. After Lemma 4.5 and Proposition 3.9 (see also Proposition 4.8, Remark 4.7 and formulas (4.20), (4.51)), we know that for any $k \in \mathbb{N}$ the operators $\left[\lambda_{\chi, \delta}^{m-1, \gamma}(Z), L_{t a n}\right] \widetilde{\lambda}_{\delta}^{-m+1, \gamma}(Z)$ and $q_{m, \delta}(x, Z, \gamma) \mathcal{L}^{I} \widetilde{\lambda}_{\delta}^{-m+1, \gamma}(Z)$ extend as linear bounded mappings from $H_{\text {tan, },}^{k}(Q)$ into itself, and their operator norms are uniformly bounded with respect to $\gamma$ and $\delta$. Combining with the result of Lemma 4.3, it follows that a positive constant $C_{s}>0$, independent of $\gamma$ and $\delta$, can be found in such a way that

$$
\left\|\tau_{m, \delta}(x, Z, \gamma) u\right\|_{H_{t a n, \gamma}^{1}(Q)} \leq C \gamma\|u\|_{L^{2}(Q)} .
$$

Concerning now the term $\left(\lambda_{\chi, \delta}^{m-1, \gamma}(Z)-\eta_{m, \delta}(x, Z, \gamma)\right) F$, after Lemma 4.5 and Proposition 4.8 we already know that the symbol $\eta_{m, \delta}(x, \xi, \gamma)$ has order $m-2$ and obeys 
the same decay estimates as in (4.22). From (3.13) then follows that $\left\{\eta_{m, \delta}\right\}_{0<\delta \leq 1}$ is a bounded subset of $\Gamma^{m-1}$; because $\left\{\lambda_{\chi, \delta}^{m-1, \gamma}\right\}_{0<\delta \leq 1}$ is also a bounded subset of $\Gamma^{m}$ (as a consequence of (4.10) and (3.13) again), after Proposition 27 in [17] we conclude that there exists a positive constant $C$, independent of $\gamma$ and $\delta$, such that

$$
\left\|\left(\lambda_{\chi, \delta}^{m-1, \gamma}(Z)-\eta_{m, \delta}(x, Z, \gamma)\right) F\right\|_{H_{t a n, \gamma}^{1}(Q)} \leq C\|F\|_{H_{t a n, \gamma}^{m+1}(Q)} .
$$

As regards to the boundary datum $b_{m, \delta}^{\prime}\left(D^{\prime}, \gamma\right) G$ in (4.69), the family of symbols $\left\{b_{m, \delta}^{\prime}\right\}_{0<\delta \leq 1}$ in $\Sigma$ defines a bounded subset of $\Gamma^{m}$; this follows from estimates (4.62) and the inequality (3.13) (in dimension $n$ ). Therefore, Proposition 3.8 (for symbols in $\Sigma$ ) implies the existence of a positive constant $C$, independent of $\gamma$ and $\delta$, such that:

$$
\left\|b_{m, \delta}^{\prime}\left(D^{\prime}, \gamma\right) G\right\|_{H_{\gamma}^{1}(\Sigma)} \leq C\|G\|_{H_{\gamma}^{m+1}(\Sigma)} .
$$

From the assumption (4) made about the $\operatorname{BVP}(4.5), \lambda_{\chi, \delta}^{m-1, \gamma}(Z) u$ is the unique $L^{2}$-solution to (4.68)-(4.69). Then we may find positive constants $\widetilde{\gamma}_{m}, \widetilde{C}_{m}$ such that for all $\gamma \geq \widetilde{\gamma}_{m}$ and $\left.\left.\delta \in\right] 0,1\right]$

$$
\begin{aligned}
& \gamma\left\|\lambda_{\chi, \delta}^{m-1, \gamma}(Z) u\right\|_{L^{2}(Q)}^{2}+\left\|\left(\lambda_{\chi, \delta}^{m-1, \gamma}(Z) u^{I}\right)_{\mid x_{1}=0}\right\|_{L^{2}(\Sigma)}^{2} \\
& \leq \widetilde{C}_{m}\left(\frac{1}{\gamma^{3}}\left\|\left(\lambda_{\chi, \delta}^{m-1, \gamma}(Z)-\eta_{m, \delta}(x, Z, \gamma)\right) F\right\|_{H_{t a n, \gamma}^{1}(Q)}^{2}\right. \\
& \left.+\frac{1}{\gamma^{2}}\left\|b_{m, \delta}^{\prime}\left(D^{\prime}, \gamma\right) G\right\|_{H_{\gamma}^{1}(\Sigma)}^{2}+\frac{1}{\gamma}\|u\|_{L^{2}(Q)}^{2}\right) \\
& \leq \widetilde{C}_{m}\left(\frac{1}{\gamma^{3}}\|F\|_{H_{t a n, \gamma}^{m+1}(Q)}^{2}+\frac{1}{\gamma^{2}}\|G\|_{H_{\gamma}^{m+1}(\Sigma)}^{2}\right) .
\end{aligned}
$$

The estimate (4.6) is used to majorize the $L^{2}$-norm of $u$ in the middle line of (4.73).

Since the quantity in the last line of (4.73) is independent of $\delta$, the $L^{2}$-norms $\left\|\lambda_{\chi, \delta}^{m-1, \gamma}(Z) u\right\|_{L^{2}(Q)}$ are bounded by (4.73) uniformly with respect to $\left.\left.\delta \in\right] 0,1\right]$. Then Corollary 4.4 gives that $u \in H_{\text {tan }, \gamma}^{m}(Q)$.

As to the Sobolev regularity of the trace on the boundary of the noncharacteristic component $u^{I}$, in view of the identity (4.66) the estimate (4.73) also gives a uniform bound, with respect to $\delta \in] 0,1]$, of $\left\|\left(\lambda_{\chi, \delta}^{m-1, \gamma}(Z) u^{I}\right)_{\mid x_{1}=0}\right\|_{L^{2}(\Sigma)}=$ $\left\|b_{m, \delta}^{\prime}\left(D^{\prime}, \gamma\right)\left(u_{\mid x_{1}=0}^{I}\right)\right\|_{L^{2}(\Sigma)}$. Then $u_{\mid x_{1}=0}^{I} \in H_{\gamma}^{m}(\Sigma)$ can be derived from the next result, the proof of which will be given in [17].

Lemma 4.12. For $m \in \mathbb{N}$ and $\delta \in] 0,1]$, let $b_{m, \delta}^{\prime}\left(\xi^{\prime}, \gamma\right)$ be defined by (4.61). Then there exists a symbol $\beta_{m, \delta}\left(\xi^{\prime}, \gamma\right) \in \Gamma^{m-2}$ such that:

$$
b_{m, \delta}^{\prime}\left(\xi^{\prime}, \gamma\right)=\lambda_{\delta}^{m-1, \gamma}\left(\xi^{\prime}\right)+\beta_{m, \delta}\left(\xi^{\prime}, \gamma\right), \quad \forall \xi^{\prime} \in \mathbb{R}^{n} .
$$

In addition, the symbol $\beta_{m, \delta}$ satisfies the following estimates: for every $\alpha^{\prime} \in \mathbb{N}^{n}$ there exists a positive constant $C_{m, \alpha^{\prime}}$, independent of $\gamma$ and $\delta$, such that

$$
\left|\partial_{\xi^{\prime}}^{\alpha^{\prime}} \beta_{m, \delta}\left(\xi^{\prime}, \gamma\right)\right| \leq C_{m, \alpha^{\prime}} \lambda_{\delta}^{m-2-\left|\alpha^{\prime}\right|, \gamma}\left(\xi^{\prime}\right), \quad \forall \xi^{\prime} \in \mathbb{R}^{n} .
$$

Arguing as was done to derive Corollary 4.4 from Lemma 4.3, from Lemma 4.12 we deduce the following 
Corollary 4.13. For every positive integer $m$ and $\gamma \geq 1, v \in H_{\gamma}^{m}(\Sigma)$ if and only if $v \in H_{\gamma}^{m-1}(\Sigma)$ and the set $\left\{\left\|b_{m, \delta}^{\prime}\left(D^{\prime}, \gamma\right) v\right\|_{L^{2}(\Sigma)}\right\}_{0<\delta \leq 1}$ is bounded.

After the result of Corollary 4.13, we conclude that $u_{\mid x_{1}=0}^{I} \in H_{\gamma}^{m}(\Sigma)$.

From estimate (4.73) and the use of the identities (4.13) and (4.74), we also get

$$
\begin{aligned}
& \gamma\left\|\lambda_{\delta}^{m-1, \gamma}(Z) u\right\|_{L^{2}(Q)}^{2}+\left\|\lambda_{\delta}^{m-1, \gamma}\left(D^{\prime}\right)\left(u_{\mid x_{1}=0}^{I}\right)\right\|_{L^{2}(\Sigma)}^{2} \\
& \leq C_{m}^{\prime}\left(\frac{1}{\gamma^{3}}\|F\|_{H_{t a n, \gamma}^{m+1}(Q)}^{2}+\frac{1}{\gamma^{2}}\|G\|_{H_{\gamma}^{m+1}(\Sigma)}^{2}\right) \\
& +2\left(\gamma\left\|r_{m, \delta}(Z, \gamma) u\right\|_{L^{2}(Q)}^{2}+\left\|\beta_{m, \delta}\left(D^{\prime}, \gamma\right)\left(u_{\mid x_{1}=0}^{I}\right)\right\|_{L^{2}(\Sigma)}^{2}\right),
\end{aligned}
$$

where the positive constant $C_{m}^{\prime}$ is again independent of $\gamma$ and $\delta$. On the other hand, using Lemma 4.3 and that $\left\{\beta_{m, \delta}\right\}_{0<\delta \leq 1}$ is a bounded subset of $\Gamma^{m-1}$ (that follows at once from Lemma 4.12 and inequality (3.13)), one can estimate

$$
\begin{aligned}
& \gamma\left\|r_{m, \delta}(Z, \gamma) u\right\|_{L^{2}(Q)}^{2}+\left\|\beta_{m, \delta}\left(D^{\prime}, \gamma\right)\left(u_{\mid x_{1}=0}^{I}\right)\right\|_{L^{2}(\Sigma)}^{2} \\
& \leq C_{m}\left(\gamma\|u\|_{L^{2}(Q)}^{2}+\left\|u_{\mid x_{1}=0}^{I}\right\|_{H_{\gamma}^{m-1}(\Sigma)}^{2}\right)
\end{aligned}
$$

with positive constant $C_{m}$ independent, once again, of $\gamma$ and $\delta$.

In the end, combining (4.76), (4.77) and using the a priori estimate (4.7) of order $m-1$ on $u$, which holds true by the inductive assumption, we conclude that there exist constants $C_{m}^{\prime \prime}>0$ and $\gamma_{m}^{\prime} \geq 1$ sufficiently large such that

$$
\begin{aligned}
& \gamma\left\|\lambda_{\delta}^{m-1, \gamma}(Z) u\right\|_{L^{2}(Q)}^{2}+\left\|\lambda_{\delta}^{m-1, \gamma}\left(D^{\prime}\right)\left(u_{\mid x_{1}=0}^{I}\right)\right\|_{L^{2}(\Sigma)}^{2} \\
& \leq C_{m}^{\prime \prime}\left(\frac{1}{\gamma^{3}}\|F\|_{H_{t a n, \gamma}^{m+1}(Q)}^{2}+\frac{1}{\gamma^{2}}\|G\|_{H_{\gamma}^{m+1}(\Sigma)}^{2}\right)
\end{aligned}
$$

for all $\gamma \geq \gamma_{m}^{\prime}$ and $\left.\left.\delta \in\right] 0,1\right]$.

The energy estimate (4.4) of order $m$ follows by letting $\delta \rightarrow 0$ into the left-hand side of (4.78) (for an arbitrarily fixed $\gamma \geq \gamma_{m}^{\prime}$ ) and exploiting the results of Propositions 3.1 and 3.2 .

\section{The IBVP. Proof for $m=1$}

Let us define the following space

$$
V_{\text {tan, } \gamma}^{2}\left(Q_{T}\right)=\left\{F \in H_{\text {tan }, \gamma}^{2}\left(Q_{T}\right): F_{\mid t=0} \in H_{\gamma}^{1}\left(\mathbb{R}_{+}^{n}\right)\right\},
$$

equipped with its natural norm. The main aim of this section is to prove the following theorem.

Theorem 5.1. Assume $\left(S_{0}, A_{i}, B\right) \in \mathcal{C}_{T}\left(H_{*, \gamma}^{\sigma}\right) \times \mathcal{C}_{T}\left(H_{*, \gamma}^{\sigma}\right) \times \mathcal{C}_{T}\left(H_{*, \gamma}^{\sigma-2}\right)$, for $i=$ $1, \ldots, n$, where $\sigma \geq[(n+1) / 2]+6$, and that problem (1.1)-(1.3) obeys the assumptions (1)-(5). Then for all $F \in V_{\text {tan, } \gamma}^{2}\left(Q_{T}\right), G \in H_{\gamma}^{2}\left(\Sigma_{T}\right), f \in H_{\gamma}^{2}\left(\mathbb{R}_{+}^{n}\right)$, satisfying the compatibility conditions $M f=G_{\mid t=0}$, and $M f^{(1)}=\partial_{t} G_{\mid t=0}$ on $\mathbb{R}^{n-1}$, the unique solution $u$ to (1.1)-(1.3), with data $(F, G, f)$, belongs to $\mathcal{C}_{T}\left(H_{*, \gamma}^{1}\right)$ and $u_{\mid \Sigma_{T}}^{I} \in H_{\gamma}^{1}\left(\Sigma_{T}\right)$. 
Moreover, there exist constants $\gamma_{1}>0, C_{1}>0$, such that for all $\gamma \geq \gamma_{1}$ u satisfies the a priori estimate

$$
\begin{aligned}
& \gamma\left\|u_{\gamma}\right\|_{H_{*, \gamma}^{1}\left(Q_{T}\right)}^{2}+\max _{t \in[0, T]} \mid\left\|u_{\gamma}(t)\right\|\left\|_{1, *, \gamma}^{2}+\right\| u_{\gamma \mid \Sigma_{T}}^{I} \|_{H_{\gamma}^{1}\left(\Sigma_{T}\right)}^{2} \\
& \leq C_{1}\left(\frac{1}{\gamma^{2}}\|f \mid\|_{2, \tan , \gamma}^{2}+\frac{1}{\gamma^{3}}\left\|F_{\gamma}\right\|_{H_{\text {tan, }}^{2}\left(Q_{T}\right)}^{2}+\frac{1}{\gamma^{2}}\left\|G_{\gamma}\right\|_{H_{\gamma}^{2}\left(\Sigma_{T}\right)}^{2}\right) .
\end{aligned}
$$

The constant $C_{1}>0$ depends on $\left\|S_{0}\right\|_{W^{2, \infty}\left(Q_{T}\right)},\left\|A_{j}\right\|_{W^{2, \infty}\left(Q_{T}\right)},\|B\|_{W^{1, \infty}\left(Q_{T}\right)}$, $\left\|\Gamma_{\beta}\right\|_{W^{1, \infty}\left(Q_{T}\right)},\|\Psi\|_{W^{1, \infty}\left(Q_{T}\right)},\left\|\Gamma_{0}\right\|_{W^{1, \infty}\left(Q_{T}\right)}$, see $(5.6)$.

We notice that Theorem 5.1 yields directly Theorem 1.2 for $m=1$ because $H_{*, \gamma}^{2}\left(Q_{T}\right) \hookrightarrow H_{\text {tan }, \gamma}^{2}\left(Q_{T}\right)$.

As a first step of the proof, we approximate the data with regularized functions satisfying the same compatibility conditions.

Lemma 5.2. Assume that problem (1.1)-(1.3) obeys the assumptions (1)-(3). Let $F \in V_{\text {tan, } \gamma}^{2}\left(Q_{T}\right), G \in H_{\gamma}^{2}\left(\Sigma_{T}\right), f \in H_{\gamma}^{2}\left(\mathbb{R}_{+}^{n}\right)$, such that $M f=G_{\mid t=0}$, and $M f^{(1)}=$ $\partial_{t} G_{\mid t=0}$ on $\mathbb{R}^{n-1}$.

Then there exist $F_{k} \in H_{\gamma}^{3}\left(Q_{T}\right), G_{k} \in H_{\gamma}^{3}\left(\Sigma_{T}\right), f_{k} \in H_{\gamma}^{3}\left(\mathbb{R}_{+}^{n}\right)$, such that $M f_{k}=G_{k \mid t=0}, M f_{k}^{(1)}=\partial_{t} G_{k \mid t=0}$ on $\mathbb{R}^{n-1}$ for every $k$, and such that $F_{k} \rightarrow F$ in $H_{t a n, \gamma}^{2}\left(Q_{T}\right), F_{k \mid t=0} \rightarrow F_{\mid t=0}$ in $H_{\gamma}^{1}\left(\mathbb{R}_{+}^{n}\right), G_{k} \rightarrow G$ in $H_{\gamma}^{2}\left(\Sigma_{T}\right), f_{k}^{(i)} \rightarrow f^{(i)}$ in $H_{\gamma}^{2-i}\left(\mathbb{R}_{+}^{n}\right)$ for $i=0,1,2$, as $k \rightarrow+\infty$.

Proof. For noncharacteristic homogeneous $(G=0)$ boundary conditions and the statement in standard Sobolev spaces $H^{m}$, a similar proposition has been proved in [22, Lemma 3.3]. Then it has been adapted to characteristic boundaries in $[2,20]$, again in standard Sobolev spaces $H^{m}$. In $H_{*}^{m}$ spaces it seems that this can be done only for $m=1$, see [25, Lemma 5.1]. The present adaptation to the nonhomogeneous case $(G \neq 0)$ follows the same lines of the proof of [25, Lemma 5.1], so we will omit the details.

Remark 5.3. Given the a priori estimate (5.1), it is natural to try to prove Theorem 5.1 under the weaker assumption $f \in H_{\text {tan, } \gamma}^{2}\left(\mathbb{R}_{+}^{n}\right)$, instead of $f \in H_{\gamma}^{2}\left(\mathbb{R}_{+}^{n}\right)$. Unfortunately, if we assume that, then we can find functions $f_{k}$ as in Lemma 5.2 which satisfy $f_{k}^{(i)} \rightarrow f^{(i)}$ in $H_{t a n, \gamma}^{2-i}\left(\mathbb{R}_{+}^{n}\right)$ for $i=0,1$, but we are not able to prove $f_{k}^{(2)} \rightarrow f^{(2)}$ in $L^{2}\left(\mathbb{R}_{+}^{n}\right)$, the latter fact being needed for our density argument, see (5.10).

Proof of Theorem 5.1. First we assume that the matrices $S_{0}, A_{j}, B$ are of $C^{\infty}$-class ${ }^{\mathrm{c}}$. Given the functions $F_{k}, G_{k}, f_{k}$ as in Lemma 5.2, we first calculate through equations

${ }^{\mathrm{c}}$ The coefficients $S_{0}, A_{j}, B$, if originally defined on $Q_{T}$, may be extended to all $Q$ in order to maintain the same regularity as in Theorem 5.1, and the properties of the block-wise structure as in (2). 
$L u=F_{k}, u_{\mid t=0}=f_{k}$, the initial time derivatives $f_{k}^{(1)} \in H_{\gamma}^{2}\left(\mathbb{R}_{+}^{n}\right)$ and $f_{k}^{(2)} \in H_{\gamma}^{1}\left(\mathbb{R}_{+}^{n}\right)$. Then we take a function $w_{k} \in H_{\gamma}^{3}\left(Q_{T}\right)$ such that

$$
w_{k \mid t=0}=f_{k}, \quad \partial_{t} w_{k \mid t=0}=f_{k}^{(1)}, \quad \partial_{t t}^{2} w_{k \mid t=0}=f_{k}^{(2)} .
$$

Notice that by definition of $f_{k}^{(1)}, f_{k}^{(2)}$ this yields

$$
\left(L w_{k}\right)_{\mid t=0}=F_{k \mid t=0}, \quad \partial_{t}\left(L w_{k}\right)_{\mid t=0}=\partial_{t} F_{k \mid t=0} .
$$

Now we look for an approximated solution $u_{k}$ of (1.1)-(1.3) with data $F_{k}, G_{k}, f_{k}$, of the form $u_{k}=v_{k}+w_{k}$, where $v_{k}$ is solution to

$$
\begin{array}{ll}
L v_{k}=F_{k}-L w_{k}, & \text { in } Q_{T} \\
M v_{k}=G_{k}-M w_{k}, & \text { on } \Sigma_{T} \\
v_{k \mid t=0}=0, & \text { in } \mathbb{R}_{+}^{n} .
\end{array}
$$

Let us denote again $L_{\gamma}=\gamma+L, u_{k \gamma}=e^{-\gamma t} u_{k}, v_{k \gamma}=e^{-\gamma t} v_{k}$ and so on. Then (5.3) is equivalent to

$$
\begin{array}{ll}
L_{\gamma} v_{k \gamma}=F_{k \gamma}-L_{\gamma} w_{k \gamma}, & \text { in } Q_{T} \\
M v_{k \gamma}=G_{k \gamma}-M w_{k \gamma}, & \text { on } \Sigma_{T} \\
v_{k \gamma \mid t=0}=0, & \text { in } \mathbb{R}_{+}^{n} .
\end{array}
$$

We easily verify that (5.2) yields

$$
\left(F_{k \gamma}-L_{\gamma} w_{k \gamma}\right)_{\mid t=0}=0, \quad \partial_{t}\left(F_{k \gamma}-L_{\gamma} w_{k \gamma}\right)_{\mid t=0}=0,
$$

and $M f_{k \mid x_{1}=0}=G_{k \mid t=0}, M f_{k \mid x_{1}=0}^{(1)}=\partial_{t} G_{k \mid t=0}$ yield

$$
\left(G_{k \gamma}-M w_{k \gamma}\right)_{\mid t=0}=0, \quad \partial_{t}\left(G_{k \gamma}-M w_{k \gamma}\right)_{\mid t=0}=0 .
$$

Thus the sufficient conditions (4.3) of Theorem 4.1 hold for $h=0,1$. We also notice that $F_{k \gamma}-L_{\gamma} w_{k \gamma} \in H_{\gamma}^{2}\left(Q_{T}\right)$ and $G_{k \gamma}-M w_{k \gamma} \in H_{\gamma}^{5 / 2}\left(\Sigma_{T}\right) \hookrightarrow H_{\gamma}^{2}\left(\Sigma_{T}\right)$. We may apply Theorem 4.1 for $\gamma$ large enough, and find $v_{k} \in H_{\text {tan, } \gamma}^{1}\left(Q_{T}\right)=H_{*, \gamma}^{1}\left(Q_{T}\right)$, with $v_{k \mid \Sigma_{T}}^{I} \in H_{\gamma}^{1}\left(\Sigma_{T}\right)$. Accordingly we infer $u_{k} \in H_{*, \gamma}^{1}\left(Q_{T}\right)$, with $u_{k \mid \Sigma_{T}}^{I} \in H_{\gamma}^{1}\left(\Sigma_{T}\right)$. Moreover $u_{k}$ solves

$$
\begin{array}{ll}
L u_{k}=F_{k}, & \text { in } Q_{T} \\
M u_{k}=G_{k}, & \text { on } \Sigma_{T} \\
u_{k \mid t=0}=f_{k}, & \text { in } \mathbb{R}_{+}^{n} .
\end{array}
$$

We look for the problem solved by $Z u_{k}=\left(Z_{1} u_{k}, \ldots, Z_{n+1} u_{k}\right) \in L^{2}\left(Q_{T}\right)$ (where $\left.Z_{n+1}=\partial_{t}\right)$. As already observed by Rauch [21], there exist matrices $\Gamma_{\beta}, \Gamma_{0}, \Psi$ such that

$$
\left[L, Z_{i}\right]=-\sum_{|\beta|=1} \Gamma_{\beta} Z^{\beta}+\Gamma_{0}+\Psi L, \quad i=1, \ldots, n+1 .
$$

As shown in [18, Lemma 41], see Lemma Appendix B.9, $\Gamma_{\beta}$ looses at most one normal and one tangential derivative w.r.t. the $A_{j}$ 's (i.e. a weight 3 in $H_{*, \gamma}^{m}$ spaces) and $\Gamma_{0}, \Psi$ loose at most one tangential derivative (weight 1 in $H_{*, \gamma}^{m}$ spaces). However, for smooth matrices $A_{j}, B$ we do not need to care about that loss of regularity. 
Applying the operators $Z_{i}$ to (5.5) and taking account of (5.6), we infer that $Z u_{k}$ solves problem

$$
\begin{array}{ll}
L Z_{i} u_{k}+\sum_{|\beta|=1} \Gamma_{\beta} Z^{\beta} u_{k}=\left(Z_{i}+\Psi\right) F_{k}+\Gamma_{0} u_{k}, & \text { in } Q_{T}, \\
M Z_{i} u_{k}=Z_{i} G_{k}, & \text { on } \Sigma_{T}, \\
Z_{i} u_{k \mid t=0}=Z_{i} f_{k}, & \text { in } \mathbb{R}_{+}^{n} .
\end{array}
$$

Applying the a priori estimate (1.11) to a difference of solutions $u_{k}-u_{h}$ of problems (5.5) readily gives

$$
\begin{aligned}
& \gamma\left\|\left(u_{k}-u_{h}\right)_{\gamma}\right\|_{L^{2}\left(Q_{t}\right)}^{2}+\left\|\left(u_{k}-u_{h}\right)_{\gamma}(t)\right\|_{L^{2}\left(\mathbb{R}_{+}^{n}\right)}^{2}+\left\|\left(u_{k}-u_{h}\right)_{\gamma \mid \Sigma_{T}}^{I}\right\|_{L^{2}\left(\Sigma_{t}\right)}^{2} \\
& \leq C_{0}\left(\frac{1}{\gamma^{3}}\left\|\left(F_{k}-F_{h}\right)_{\gamma}\right\|_{H_{t a n, \gamma}^{1}\left(Q_{t}\right)}^{2}+\frac{1}{\gamma^{2}} \mid\left\|f_{k}-f_{h}\right\|\left\|_{1, t a n, \gamma}^{2}+\frac{1}{\gamma^{2}}\right\|\left(G_{k}-G_{h}\right)_{\gamma} \|_{H_{\gamma}^{1}\left(\Sigma_{t}\right)}^{2}\right) .
\end{aligned}
$$

Applying the a priori estimate (1.11) also to a difference of solutions $Z u_{k}-Z u_{h}$ of problems (5.7) gives

$$
\begin{aligned}
& \gamma\left\|\left(Z u_{k}-Z u_{h}\right)_{\gamma}\right\|_{L^{2}\left(Q_{t}\right)}^{2}+\left\|\left(Z u_{k}-Z u_{h}\right)_{\gamma}(t)\right\|_{L^{2}\left(\mathbb{R}_{+}^{n}\right)}^{2}+\left\|\left(Z u_{k}-Z u_{h}\right)_{\gamma \mid \Sigma_{T}}^{I}\right\|_{L^{2}\left(\Sigma_{t}\right)}^{2} \\
& \leq C_{0}\left(\frac{1}{\gamma^{3}}\left\|\left((Z+\Psi)\left(F_{k}-F_{h}\right)\right)_{\gamma}+\Gamma_{0}\left(u_{k}-u_{h}\right)_{\gamma}\right\|_{H_{t a n, \gamma}^{1}\left(Q_{t}\right)}^{2}\right. \\
& \left.+\frac{1}{\gamma^{2}}\left\|Z f_{k}-Z f_{h}\right\|\left\|_{1, t a n, \gamma}^{2}+\frac{1}{\gamma^{2}}\right\|\left(Z G_{k}-Z G_{h}\right)_{\gamma} \|_{H_{\gamma}^{1}\left(\Sigma_{t}\right)}^{2}\right) \\
& \leq C_{0}^{\prime}\left(\frac{1}{\gamma^{3}}\left\|\left(F_{k}-F_{h}\right)_{\gamma}\right\|_{H_{t a n, \gamma}^{2}}^{2}\left(Q_{t}\right)+\frac{1}{\gamma^{3}}\left\|\left(u_{k}-u_{h}\right)_{\gamma}\right\|_{H_{t a n, \gamma}^{1}\left(Q_{t}\right)}^{2}\right. \\
& \left.+\frac{1}{\gamma^{2}}\left\|f_{k}-f_{h}\right\|\left\|_{2, t a n, \gamma}^{2}+\frac{1}{\gamma^{2}}\right\|\left(G_{k}-G_{h}\right)_{\gamma} \|_{H_{\gamma}^{2}\left(\Sigma_{t}\right)}^{2}\right) .
\end{aligned}
$$

From (5.8) and (5.9) we then get for $\gamma$ large enough and a suitable constant $C_{1}$

$$
\begin{aligned}
& \gamma\left\|\left(u_{k}-u_{h}\right)_{\gamma}\right\|_{H_{*, \gamma}^{1}\left(Q_{t}\right)}^{2}+\left\|\mid\left(u_{k}-u_{h}\right)_{\gamma}(t)\right\|\left\|_{1, *, \gamma}^{2}+\right\|\left(u_{k}-u_{h}\right)_{\gamma \mid \Sigma_{T}}^{I} \|_{H_{\gamma}^{1}\left(\Sigma_{t}\right)}^{2} \\
& \leq C_{1}\left(\frac{1}{\gamma^{3}}\left\|\left(F_{k}-F_{h}\right)_{\gamma}\right\|_{H_{t a n, \gamma}^{2}}^{2}\left(Q_{t}\right)+\frac{1}{\gamma^{2}}\left\|f_{k}-f_{h}\right\|_{2, t a n, \gamma}^{2}+\frac{1}{\gamma^{2}}\left\|\left(G_{k}-G_{h}\right)_{\gamma}\right\|_{H_{\gamma}^{2}\left(\Sigma_{t}\right)}^{2}\right) .
\end{aligned}
$$

We remark that the constant $C_{0}^{\prime}$ in (5.9) depends on $\left\|S_{0}\right\|_{W^{2, \infty}\left(Q_{T}\right)},\left\|A_{j}\right\|_{W^{2, \infty}\left(Q_{T}\right)}$, $\|B\|_{W^{1, \infty}\left(Q_{T}\right)}$, in the same way as $C_{0}$ in (1.9),(5.8), but also on the norm $\left\|\Gamma_{\beta}\right\|_{W^{1, \infty}\left(Q_{T}\right)}$; the constant $C_{1}$ in (5.10) incorporates $C_{0}^{\prime}$ and also depends on $\|\Psi\|_{W^{1, \infty}\left(Q_{T}\right)},\left\|\Gamma_{0}\right\|_{W^{1, \infty}\left(Q_{T}\right)}$.

From (5.10) and Lemma 5.2 we infer that $\left\{u_{k}\right\}$ is a Cauchy sequence in $\mathcal{C}_{T}\left(H_{*, \gamma}^{1}\right)$, and $\left\{u_{k \mid \Sigma_{T}}^{I}\right\}$ is a Cauchy sequence in $H_{\gamma}^{1}\left(\Sigma_{T}\right)$. Therefore there exists a function in $\mathcal{C}_{T}\left(H_{*, \gamma}^{1}\right)$ which is the limit of $\left\{u_{k}\right\}$. Passing to the limit in (5.5) as $k \rightarrow \infty$, we see that this function is a solution to (1.1)-(1.3). The uniqueness of the $L^{2}$ solution yields $u \in \mathcal{C}_{T}\left(H_{*, \gamma}^{1}\right)$ and $u_{\mid \Sigma_{T}}^{I} \in H_{\gamma}^{1}\left(\Sigma_{T}\right)$. Applying the a priori estimate (1.11) to the solutions $u_{k}, Z_{i} u_{k}$ of problems (5.5), (5.7), with calculations as above, gives (5.1) for $u_{k}$. Passing to the limit in $k$ finally gives (5.1) for $u$.

Up to now we have considered matrices $S_{0}, A_{j}, B$ of $C^{\infty}$-class. Now we wish to solve the problem with coefficients with finite regularity as in Theorem 5.1, by a density argument. 
Given matrices $S_{0}, A_{j}, B$ with the properties prescribed in the statement of Theorem 5.1, let us take approximating sequences $S_{0}^{(k)}, A_{j}^{(k)}, B^{(k)}$ in $C_{(0)}^{\infty}$, such that $S_{0}^{(k)} \rightarrow S_{0}, A_{j}^{(k)} \rightarrow A_{j}$ in $\mathcal{C}_{T}\left(H_{*, \gamma}^{\sigma}\right)$, and $B^{(k)} \rightarrow B$ in $\mathcal{C}_{T}\left(H_{*, \gamma}^{\sigma-2}\right)$, as $k \rightarrow \infty$, where $\sigma \geq[(n+1) / 2]+6$. We may also assume that $S_{0}^{(k)}$ is definite positive, and that the new boundary matrix has the same properties as in (2). This yields operators $L^{(k)}$ converging to $L$, where assumptions (1)-(4) are still satisfied. This is the point where we use assumption (5), in order to get (4) also for $L^{(k)}$.

In this context now we prove the following result. To avoid overloading with the introduction of a new notation, we use the same symbols of Lemma 5.2.

Lemma 5.4. Let $F, G, f$ be given as in the statement of Theorem 5.1. There exist $F_{k} \in H_{\gamma}^{3}\left(Q_{T}\right), G_{k} \in H_{\gamma}^{3}\left(\Sigma_{T}\right), f_{k} \in H_{\gamma}^{3}\left(\mathbb{R}_{+}^{n}\right)$, such that $M f_{k}=G_{k \mid t=0}, M f_{k}^{(1)}=$ $\partial_{t} G_{k \mid t=0}$ on $\mathbb{R}^{n-1}$ for every $k$, and such that $F_{k} \rightarrow F$ in $H_{t a n, \gamma}^{2}\left(Q_{T}\right), F_{k \mid t=0} \rightarrow F_{\mid t=0}$ in $H_{\gamma}^{1}\left(\mathbb{R}_{+}^{n}\right), G_{k} \rightarrow G$ in $H_{\gamma}^{2}\left(\Sigma_{T}\right), f_{k}^{(i)} \rightarrow f^{(i)}$ in $H_{\gamma}^{2-i}\left(\mathbb{R}_{+}^{n}\right)$ for $i=0,1,2$, as $k \rightarrow+\infty$, where now $f_{k}^{(1)}$ and $f_{k}^{(2)}$ are defined by

$$
\begin{gathered}
f_{k}^{(1)}+\sum_{i=1}^{n} A_{i \mid t=0}^{(k)} \partial_{i} f_{k}+B_{\mid t=0}^{(k)} f_{k}=F_{k \mid t=0} \\
f_{k}^{(2)}+\sum_{i=1}^{n} A_{i \mid t=0}^{(k)} \partial_{i} f_{k}^{(1)}+\sum_{i=1}^{n} \partial_{t} A_{i \mid t=0}^{(k)} \partial_{i} f_{k}+B_{\mid t=0}^{(k)} f_{k}^{(1)}+\partial_{t} B_{\mid t=0}^{(k)} f_{k}=\partial_{t} F_{k \mid t=0} .
\end{gathered}
$$

Proof. The proof is quite similar to that of Lemma 5.2, so we refer again to [25, Lemma 5.1].

We consider the problems

$$
\begin{array}{ll}
L^{(k)} u^{(k)}=F_{k}, & \text { in } Q_{T} \\
M u^{(k)}=G_{k}, & \text { on } \Sigma_{T} \\
u_{\mid t=0}^{(k)}=f_{k}, & \text { in } \mathbb{R}_{+}^{n} .
\end{array}
$$

The operator $L^{(k)}$ has $C^{\infty}$ coefficients and the data have the required regularity and enjoy the compatibility condition of order 0 and 1 . Therefore we may apply the previous step of the proof and find solutions $u^{(k)} \in \mathcal{C}_{T}\left(H_{*, \gamma}^{1}\right)$ with $u_{\mid \Sigma_{T}}^{I(k)} \in H_{\gamma}^{1}\left(\Sigma_{T}\right)$. Since $S_{0}^{(k)}, A_{j}^{(k)}$ are uniformly bounded in $\mathcal{C}_{T}\left(H_{*, \gamma}^{[(n+1) / 2]+5}\right)$, applying the imbedding Theorem Appendix B.2 shows that they are uniformly bounded in $C^{0}\left([0, T] ; W^{2, \infty}\right) \cap C^{1}\left([0, T] ; W^{1, \infty}\right) \cap C^{2}\left([0, T] ; L^{\infty}\right)$, and therefore in $W^{2, \infty}\left(Q_{T}\right)$. Similarly one infers that the $B^{(k)}$ 's are uniformly bounded in $W^{1, \infty}\left(Q_{T}\right)$.

Again, since $S_{0}^{(k)}, A_{j}^{(k)}$ are uniformly bounded in $\mathcal{C}_{T}\left(H_{*, \gamma}^{[(n+1) / 2]+6}\right)$ as well, by Lemma Appendix B.9 the approximating matrices $\Gamma_{\beta}^{(k)}$ are uniformly bounded in $C\left([0, T] ; H_{*, \gamma}^{[(n+1) / 2]+3}\right)$. We may apply the imbedding Theorem Appendix B.2 and obtain the uniform boundedness in $W^{1, \infty}\left(Q_{T}\right)$. Similarly we infer the uniform boundedness in $W^{1, \infty}\left(Q_{T}\right)$ of $\Gamma_{0}^{(k)}, \Psi^{(k)}$. 
Then the $u^{(k)}$ 's satisfy the a priori estimate (5.1) with uniformly bounded constants $C_{1}^{(k)}$. Therefore the sequence $\left\{u^{(k)}\right\}$ is bounded in $\mathcal{C}_{T}\left(H_{*, \gamma}^{1}\right)$ with $\left\{u_{\mid \Sigma_{T}}^{I(k)}\right\}$ bounded in $H_{\gamma}^{1}\left(\Sigma_{T}\right)$. Passing to a subsequence we get a solution $u \in$ $L^{\infty}\left(0, T ; H_{*, \gamma}^{1}\left(\mathbb{R}_{+}^{n}\right)\right) \cap W^{1, \infty}\left(0, T ; L^{2}\left(\mathbb{R}_{+}^{n}\right)\right)$ with $u_{\mid \Sigma_{T}}^{I} \in H_{\gamma}^{1}\left(\Sigma_{T}\right)$. The uniqueness of the solution yields the convergence of the whole sequence. The strong continuity in time follows by adapting Majda's approach [16]. This completes the proof of Theorem 5.1.

\section{The IBVP. Proof for $m \geq 2$}

The proof proceeds by induction. Assume that Theorem 1.2 holds up to $m-1$. Given the data $(F, G, f)$ as in Theorem 1.2, by the inductive hypothesis there exists a unique solution $u$ of problem (1.1)-(1.3) such that $u \in \mathcal{C}_{T}\left(H_{*, \gamma}^{m-1}\right)$ and $u_{\mid \Sigma_{T}}^{I} \in$ $H_{\gamma}^{m-1}\left(\Sigma_{T}\right)$.

In order to show that $u \in \mathcal{C}_{T}\left(H_{*, \gamma}^{m}\right)$, we have to increase the regularity of $u$ by order one, that is by one more tangential derivative and, if $m$ is even, also by one more normal derivative. The idea is the same as in $[24,25]$, revisited as in $[6,18,26]$. At every step we can estimate some derivatives of $u$ through equations where in the right-hand side we can put other derivatives of $u$ that have already been estimated at previous steps. The big difference is that now we have to deal with the loss of one derivative in the right-hand side. For the increase of regularity we consider the system (6.4) of equations for purely tangential derivatives, of the type of (1.1)(1.3), where we can use the inductive assumption, and other systems (6.9), (6.11) of equations for mixed tangential and normal derivatives where the boundary matrix vanishes identically, so that no boundary condition is needed and we can apply a standard energy method, under the assumption of the symmetrizable system.

When we consider the system (6.4) of equations for purely tangential derivatives, we have the loss of one derivative in the right-hand side. However the terms in the right-hand side have order $m-1$; after the loss of one derivative they become essentially of order $m$, and can be absorbed for $\gamma$ large by similar terms in the left-hand side.

From now on in this section we will assume that the system of equations (1.1) has been written in symmetric form and we write $A_{j}$ instead of $S_{0} A_{j}, B$ instead of $S_{0} B, F$ for $S_{0} F$; we also denote $A_{n+1}=S_{0}$.

\subsection{Purely tangential regularity}

Let us start by considering all the tangential derivatives $Z^{\alpha} u,|\alpha|=m-1$. We decompose $\partial_{1} u=\left(\begin{array}{c}\partial_{1} u^{I} \\ \partial_{1} u^{I I}\end{array}\right)$. By inverting $A_{1}^{I, I}$ in (1.1), we can write $\partial_{1} u^{I}$ as the sum of tangential derivatives by

$$
\partial_{1} u^{I}=\Lambda Z u+R
$$


where

$$
\begin{aligned}
& \Lambda Z u=-\left(A_{1}^{I, I}\right)^{-1}\left[\left(A_{n+1} Z_{n+1} u+\sum_{j=2}^{n} A_{j} Z_{j} u\right)^{I}+A_{1}^{I, I I} \partial_{1} u^{I I}\right], \\
& R=\left(A_{1}^{I, I}\right)^{-1}(F-B u)^{I} .
\end{aligned}
$$

Here and below, everywhere it is needed, we use the fact that, if a matrix $A$ vanishes on $\left\{x_{1}=0\right\}$, we can write $A \partial_{1} u=H Z_{1} u$, where $H$ is a suitable matrix such that $\|H\|_{H_{*}^{s-2}\left(\mathbb{R}^{n}\right)} \leq c\|A\|_{H_{*}^{s},\left(\mathbb{R}_{+}^{n}\right)}$ see Lemmata Appendix B.10 and Appendix B.11; this trick transforms some normal derivatives into tangential derivatives. We obtain $\Lambda \in \mathcal{C}_{T}\left(H_{*, \gamma}^{s-2}\right)$.

Applying the operator $Z^{\alpha}$ to (1.1), with $\alpha=\left(\alpha^{\prime}, \alpha_{n+1}\right), \alpha^{\prime}=\left(\alpha_{1}, \cdots, \alpha_{n}\right)$, and substituting (6.1) gives

$$
\begin{aligned}
& L\left(Z^{\alpha} u\right)+\sum_{|\gamma|=|\alpha|-1} \sum_{j=2}^{n+1} Z A_{j} Z_{j} Z^{\gamma} u+\sum_{|\gamma|=|\alpha|-1} Z A_{1}\left(\begin{array}{c}
\Lambda Z\left(Z^{\gamma} u\right) \\
0
\end{array}\right) \\
& -\alpha_{1} A_{1}\left(\begin{array}{c}
\Lambda Z\left(Z_{1}^{\alpha_{1}-1} Z_{2}^{\alpha_{2}} \cdots Z_{n+1}^{\alpha_{n+1}} u\right) \\
0
\end{array}\right) \\
& +\left(\sum_{|\gamma|=|\alpha|-1} Z A_{1} Z^{\gamma}-\alpha_{1} A_{1} Z_{1}^{\alpha_{1}-1} Z_{2}^{\alpha_{2}} \cdots Z_{n+1}^{\alpha_{n+1}}\right)\left(\begin{array}{c}
0 \\
\partial_{1} u^{I I}
\end{array}\right)=F_{\alpha},
\end{aligned}
$$

where

$$
\begin{aligned}
F_{\alpha}= & -\sum_{|\beta| \geq 2, \beta \leq \alpha}\left[\sum_{j=2}^{n+1} Z^{\beta} A_{j} Z_{j} Z^{\alpha-\beta} u+Z^{\beta} A_{1} Z^{\alpha-\beta}\left(\begin{array}{c}
\Lambda Z u+R \\
\partial_{1} u^{I I}
\end{array}\right)\right] \\
& -\left(\begin{array}{c}
\alpha_{1} \\
2
\end{array}\right) A_{1} Z_{1}^{\alpha_{1}-2} Z_{2}^{\alpha_{2}} \cdots Z_{n+1}^{\alpha_{n+1}}\left(\begin{array}{c}
\Lambda Z u+R \\
\partial_{1} u^{I I}
\end{array}\right) \\
& -A_{1} \partial_{1} Z_{n+1}^{\alpha_{n+1}}\left[\left(Z_{1}-1\right)^{\alpha_{1}}-Z_{1}^{\alpha_{1}}+\alpha_{1}\left(Z_{1}-1\right)^{\alpha_{1}-1}\right. \\
& \left.-\left(\begin{array}{c}
\alpha_{1} \\
2
\end{array}\right)\left(Z_{1}-1\right)^{\alpha_{1}-2}\right] Z_{2}^{\alpha_{2}} \cdots Z_{n}^{\alpha_{n}} u-\sum_{\left|\alpha^{\prime}\right|=|\alpha|-1} Z A_{1}\left[Z^{\alpha^{\prime}},\left(\begin{array}{c}
\Lambda \\
0
\end{array}\right)\right] Z u \\
& +\alpha_{1} A_{1}\left[Z_{1}^{\alpha_{1}-1} Z_{2}^{\alpha_{2}} \cdots Z_{n+1}^{\alpha_{n+1}},\left(\begin{array}{c}
\Lambda \\
0
\end{array}\right)\right] Z u-\left[Z^{\alpha}, B\right] u \\
& -\left(\sum_{\left|\alpha^{\prime}\right|=|\alpha|-1} Z A_{1} Z^{\alpha^{\prime}}-\alpha_{1} A_{1} Z_{1}^{\alpha_{1}-1} Z_{2}^{\alpha_{2}} \cdots Z_{n+1}^{\alpha_{n+1}}\right)\left(\begin{array}{c}
R \\
0
\end{array}\right)+Z^{\alpha} F .
\end{aligned}
$$

Equation (6.2) takes the form $(L+\bar{B}) Z^{\alpha} u=F_{\alpha}$ with $\bar{B} \in \mathcal{C}_{T}\left(H_{*, \gamma}^{s-3}\right)$. As for the regularity of $\bar{B}$, we notice that $s-3 \geq[(n+1) / 2]+4$, as required in Theorem 5.1 for the zero order term $B$.

Then we consider the problem satisfied by the vector of all tangential derivatives $Z^{\alpha} u$ of order $|\alpha|=m-1$. From (6.2) this problem takes the form

$$
\begin{array}{ll}
(\mathcal{L}+\mathcal{B}) Z^{\alpha} u=\mathcal{F} & \text { in } Q_{T}, \\
\mathcal{M} Z^{\alpha} u=Z^{\alpha} G & \text { on } \Sigma_{T}, \\
\left.Z^{\alpha} u\right|_{t=0}=\tilde{f} & \text { in } \mathbb{R}_{+}^{n},
\end{array}
$$


where

$$
\mathcal{L}=\left(\begin{array}{lll}
L & & \\
& \ddots & \\
& & L
\end{array}\right), \quad \mathcal{M}=\left(\begin{array}{lll}
M & & \\
& \ddots & \\
& & M
\end{array}\right)
$$

$\mathcal{B} \in \mathcal{C}_{T}\left(H_{*, \gamma}^{s-3}\right)$ is a suitable matrix and $\mathcal{F}$ is the vector of all right-hand sides $F_{\alpha}$. The initial datum $\tilde{f}$ is the vector of functions $Z^{\alpha^{\prime}} f^{\left(\alpha_{n+1}\right)}$.

Our aim is to increase the regularity of $Z^{\alpha} u$ by applying Theorem 5.1. We first observe that $G \in H_{\gamma}^{m+1}\left(\Sigma_{T}\right)$ readily yields $Z^{\alpha} G \in H_{\gamma}^{2}\left(\Sigma_{T}\right)$. Moreover the regularity of the initial data d yields $\tilde{f} \in H_{\gamma}^{2}\left(\mathbb{R}_{+}^{n}\right)$, and the data satisfy the compatibility conditions of order 0 and 1 .

If we may prove that $\mathcal{F} \in V_{\text {tan, } \gamma}^{2}\left(Q_{T}\right)$, then applying Theorem 5.1 will yield $Z^{\alpha} u \in \mathcal{C}_{T}\left(H_{*, \gamma}^{1}\right)$ with $Z^{\alpha} u_{\mid \Sigma_{T}}^{I} \in H_{\gamma}^{1}\left(\Sigma_{T}\right)$, for all $|\alpha|=m-1$. It is easily verified that the initial regularity of the data yields $\mathcal{F}_{\mid t=0} \in H_{\gamma}^{1}\left(\mathbb{R}_{+}^{n}\right)$, see (6.3). Then we estimate $\mathcal{F}$ in $H_{\text {tan, }}^{2}\left(Q_{T}\right)$, where we make use of the space $K_{*, \gamma}^{m}$, defined in (B.29), and specifically of the property about products of functions given in Theorem Appendix B.8. For the first terms in the right-hand side of (6.3) we have (hereafter $C$ is independent of $\gamma \geq 1$ )

$$
\begin{aligned}
& \sum_{j=2}^{n+1} \sum_{|\beta| \geq 2, \beta \leq \alpha}\left\|\left(Z^{\beta} A_{j} Z_{j} Z^{\alpha-\beta} u\right)_{\gamma}\right\|_{H_{t a n, \gamma}^{2}\left(Q_{t}\right)} \leq C \sum_{j=2}^{n+1} \sum_{|\delta|=2}\left\|Z^{\delta} A_{j} Z u_{\gamma}\right\|_{H_{t a n, \gamma}^{m-1}\left(Q_{t}\right)} \\
& \leq C \sum_{j=2}^{n+1} \sum_{|\delta|=2}\left\|Z^{\delta} A_{j}\right\|_{\mathcal{C}_{t}\left(H_{*, \gamma}^{s-2}\right)}\left\|Z u_{\gamma}\right\|_{K_{*, \gamma}^{m-1}\left(Q_{t}\right)} \leq C \sum_{j=2}^{n+1}\left\|A_{j}\right\|_{\mathcal{C}_{t}\left(H_{*, \gamma}^{s}\right)}\left\|u_{\gamma}\right\|_{K_{*, \gamma}^{m}\left(Q_{t}\right)} .
\end{aligned}
$$

A similar calculation gives (here we also use Theorem Appendix B.5)

$$
\begin{aligned}
\sum_{|\beta| \geq 2, \beta \leq \alpha} & \left\|Z^{\beta} A_{1} Z^{\alpha-\beta}\left(\begin{array}{c}
\Lambda Z u_{\gamma} \\
0
\end{array}\right)\right\|_{H_{t a n, \gamma}^{2}\left(Q_{t}\right)} \\
& \leq C\left\|A_{1}\right\|_{\mathcal{C}_{t}\left(H_{*, \gamma}^{s}\right)}\|\Lambda\|_{\mathcal{C}_{t}\left(H_{*, \gamma}^{s-2}\right)}\left\|u_{\gamma}\right\|_{K_{*, \gamma}^{m}\left(Q_{t}\right)} .
\end{aligned}
$$

The most critical term is (recall the decomposition $A_{1}=A_{1}^{1}+A_{1}^{2}$ in (4.17)):

$$
\begin{aligned}
& \sum_{|\beta| \geq 2, \beta \leq \alpha}\left\|Z^{\beta} A_{1}^{2} Z^{\alpha-\beta}\left(\begin{array}{c}
0 \\
\partial_{1} u_{\gamma}^{I I}
\end{array}\right)\right\|_{H_{\text {tan, }}^{2}\left(Q_{t}\right)} \leq \sum_{|\beta|=2}\left\|Z^{\beta} A_{1}^{2} Z^{\alpha-\beta}\left(\begin{array}{c}
0 \\
\partial_{1} u_{\gamma}^{I I}
\end{array}\right)\right\|_{H_{t a n, \gamma}^{2}\left(Q_{t}\right)} \\
& +\sum_{3 \leq|\beta| \leq m-2, \beta \leq \alpha}\left\|Z^{\beta} A_{1}^{2} Z^{\alpha-\beta}\left(\begin{array}{c}
0 \\
\partial_{1} u_{\gamma}^{I I}
\end{array}\right)\right\|_{H_{t a n, \gamma}^{2}\left(Q_{t}\right)}+\left\|Z^{\alpha} A_{1}^{2}\left(\begin{array}{c}
0 \\
\partial_{1} u_{\gamma}^{I I}
\end{array}\right)\right\|_{H_{t a n, \gamma}^{2}\left(Q_{t}\right)} .
\end{aligned}
$$

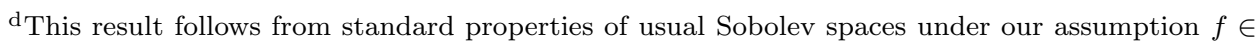
$H_{\gamma}^{m+1}\left(\mathbb{R}_{+}^{n}\right), \partial_{t}^{i} F_{\mid t=0} \in H_{\gamma}^{m-i}\left(\mathbb{R}_{+}^{n}\right)$ for $i=0, \ldots, m-1$.
} 
As for the first term in the r.h.s. of (6.5) we have

$$
\begin{aligned}
& \sum_{|\beta|=2}\left\|Z^{\beta} A_{1}^{2} Z^{\alpha-\beta}\left(\begin{array}{c}
0 \\
\partial_{1} u_{\gamma}^{I I}
\end{array}\right)\right\|_{H_{t a n, \gamma}^{2}\left(Q_{t}\right)} \leq C \sum_{|\beta|=2}\left\|Z^{\beta} H_{1} Z^{\alpha-\beta} Z_{1} u_{\gamma}\right\|_{H_{t a n, \gamma}^{2}\left(Q_{t}\right)} \\
& \leq C \sum_{|\delta|=2}\left\|Z^{\delta} H_{1}\right\|_{\mathcal{C}_{t}\left(H_{*, \gamma}^{[(n+1) / 2]+3}\right)}\left\|u_{\gamma}\right\|_{H_{t a n, \gamma}^{m}\left(Q_{t}\right)} \leq C\left\|A_{1}\right\|_{\mathcal{C}_{t}\left(H_{*, \gamma}^{s}\right)}\left\|u_{\gamma}\right\|_{K_{*, \gamma}^{m}\left(Q_{t}\right)},
\end{aligned}
$$

taking account of case $m=2$ in Theorem Appendix B.8, and Lemma Appendix B.11. For the second term in the r.h.s. of (6.5) we have

$$
\begin{aligned}
& \sum_{3 \leq|\beta| \leq m-2, \beta \leq \alpha}\left\|Z^{\beta} A_{1}^{2} Z^{\alpha-\beta}\left(\begin{array}{c}
0 \\
\partial_{1} u_{\gamma}^{I I}
\end{array}\right)\right\|_{H_{t a n, \gamma}^{2}\left(Q_{t}\right)} \\
& \leq C\left\|Z^{\beta} A_{1}^{2} Z\left(\begin{array}{c}
0 \\
\partial_{1} u_{\gamma}^{I I}
\end{array}\right)\right\|_{H_{t a n, \gamma}^{m-3}\left(Q_{t}\right)} \\
& \leq C \sum_{|\beta|=3}\left\|Z^{\beta} A_{1}\right\|_{\mathcal{C}_{t}\left(H_{*, \gamma}^{s-3}\right)}\left\|Z \partial_{1} u_{\gamma}^{I I}\right\|_{K_{*, \gamma}^{m-3}\left(Q_{t}\right)} \\
& \leq C\left\|A_{1}\right\|_{\mathcal{C}_{t}\left(H_{*, \gamma}^{s}\right)}\left(\left\|\partial_{1} u_{\gamma}^{I I}\right\|_{H_{t a n, \gamma}^{m-2}\left(Q_{t}\right)}+\gamma\left\|u_{\gamma}\right\|_{H_{*, \gamma}^{m-1}\left(Q_{t}\right)}\right) .
\end{aligned}
$$

For the last term in the r.h.s. of (6.5) we also get

$$
\left\|Z^{\alpha} A_{1}^{2}\left(\begin{array}{c}
0 \\
\partial_{1} u_{\gamma}^{I I}
\end{array}\right)\right\|_{H_{t a n, \gamma}^{2}\left(Q_{t}\right)} \leq C\left\|A_{1}\right\|_{\mathcal{C}_{t}\left(H_{*, \gamma}^{s}\right)}\left\|u_{\gamma}\right\|_{K_{*, \gamma}^{m}\left(Q_{t}\right)} .
$$

The estimate of the other terms in $\mathcal{F}$ proceeds in a similar way and in summary gives

$$
\begin{aligned}
& \left\|\mathcal{F}_{\gamma}\right\|_{H_{t a n, \gamma}^{2}\left(Q_{t}\right)} \\
& \leq C\left(\left\|u_{\gamma}\right\|_{H_{t a n, \gamma}^{m}\left(Q_{t}\right)}+\left\|\partial_{1} u_{\gamma}^{I I}\right\|_{H_{t a n, \gamma}^{m-2}\left(Q_{t}\right)}+\gamma\left\|u_{\gamma}\right\|_{H_{*, \gamma}^{m-1}\left(Q_{t}\right)}+\left\|F_{\gamma}\right\|_{H_{t a n, \gamma}^{m+1}\left(Q_{t}\right)}\right),
\end{aligned}
$$

where the constant $C$ depends on $\left\|A_{j}\right\|_{\mathcal{C}_{T}\left(H_{*, \gamma}^{s}\right)},\|B\|_{\mathcal{C}_{T}\left(H_{*, \gamma}^{s-1}\right)}$. Applying estimate (5.1) to the solution $Z^{\alpha} u$ of (6.4) and using (6.6) and (1.13) at step $m-1$ gives, for all $\gamma$ sufficiently large and $0<t \leq T$,

$$
\begin{aligned}
\gamma\left\|u_{\gamma}\right\|_{H_{t a n, \gamma}^{m}\left(Q_{t}\right)}^{2}+\|\| u_{\gamma}(t)\|\|_{m, t a n, \gamma}^{2}+\left\|u_{\gamma \mid \Sigma_{t}}^{I}\right\|_{H_{\gamma}^{m}\left(\Sigma_{t}\right)}^{2} \\
\leq C\left(\frac{1}{\gamma^{2}}\|f\| \|_{m+1, *, \gamma}^{2}+\frac{1}{\gamma^{3}}\left(\left\|F_{\gamma}\right\|_{H_{*, \gamma}^{m+1}\left(Q_{t}\right)}^{2}+\left\|\partial_{1} u_{\gamma}^{I I}\right\|_{H_{t a n, \gamma}^{m-2}\left(Q_{t}\right)}\right)\right. \\
\left.\quad+\frac{1}{\gamma^{2}}\left\|G_{\gamma}\right\|_{H_{\gamma}^{m+1}\left(\Sigma_{t}\right)}^{2}\right) .
\end{aligned}
$$

\subsection{Tangential and one normal derivatives}

We apply to the part $I I$ of (1.1) the operator $Z^{\beta} \partial_{1}$, with $|\beta|=m-2$. We obtain equation (28) in [6], that is

$$
\begin{aligned}
& {\left[\left(L+\partial_{1} A_{1}\right) Z^{\beta}+\sum_{|\gamma|=|\beta|-1}\left(Z A_{0} \partial_{t}+\sum_{j=1}^{n} Z A_{j} \partial_{j}\right) Z^{\gamma}\right.} \\
& \left.-\beta_{1} A_{1} \partial_{1} Z_{1}^{\beta_{1}-1} Z_{2}^{\beta_{2}} \cdots Z_{n+1}^{\beta_{n+1}}\right]^{I I, I I} \partial_{1} u^{I I}=\mathcal{G}
\end{aligned}
$$


where the exact expression of $\mathcal{G}$ may be found in [6]. Using (6.1) again, we write $(6.8)$ as

$$
(\tilde{\mathcal{L}}+\tilde{\mathcal{C}}) Z^{\beta} \partial_{1} u^{I I}=\mathcal{G}
$$

where

$$
\tilde{\mathcal{L}}=\left(\begin{array}{lll}
\tilde{L} & & \\
& \ddots & \\
& & \tilde{L}
\end{array}\right)
$$

with $\tilde{L}=A_{0}^{I I, I I} \partial_{t}+\sum_{j=1}^{n} A_{j}^{I I, I I} \partial_{j}$ and where $\tilde{\mathcal{C}} \in \mathcal{C}_{T}\left(H_{*, \gamma}^{s-2}\right)$ is a suitable matrix. Here a crucial point is that (6.9) is a transport-type equation, because the boundary matrix of $\tilde{\mathcal{L}}$ vanishes at $\left\{x_{1}=0\right\}$. Thus we do not need any boundary condition. Moreover, a standard energy argument gives an $L^{2}$ a priori estimate for the solution with no loss of regularity w.r.t. the source term $\mathcal{G}$. For its estimate it is important to observe that the only derivatives of $u$ of order $m$ contained in $\mathcal{G}$ are tangential derivatives, estimated in (6.7). We get the a priori estimate

$$
\begin{aligned}
& \gamma\left\|\partial_{1} u_{\gamma}^{I I}\right\|_{H_{t a n, \gamma}^{m-2}\left(Q_{t}\right)}+\|\mid\| \partial_{1} u_{\gamma}^{I I}(t)\|\|_{m-2, t a n, \gamma} \\
& \leq C\left(\|\| f \|\left.\right|_{m, *, \gamma} ^{2}+\frac{1}{\gamma}\left(\left\|F_{\gamma}\right\|_{H_{*, \gamma}^{m}\left(Q_{t}\right)}^{2}+\left\|u_{\gamma}\right\|_{H_{t a n, \gamma}^{m}\left(Q_{t}\right)}+\gamma\left\|u_{\gamma}\right\|_{H_{*, \gamma}^{m-1}\left(Q_{t}\right)}\right)\right),
\end{aligned}
$$

for all $\gamma$ sufficiently large and $0<t \leq T$.

Combining (6.7), (6.10) and applying Theorem 5.1, we infer $u \in \mathcal{C}_{T}\left(H_{t a n, \gamma}^{m}\right)$, and $u_{\mid \Sigma_{T}}^{I} \in H_{\gamma}^{m}\left(\Sigma_{T}\right)$. We also deduce that equation (6.9) has a unique solution $Z^{\beta} \partial_{1} u^{I I} \in C_{T}\left(L^{2}\right):=C\left([0, T] ; L^{2}\left(\mathbb{R}_{+}^{n}\right)\right)$, for all $|\beta|=m-2$, i.e. $\partial_{1} u^{I I} \in \mathcal{C}_{T}\left(H_{t a n, \gamma}^{m-2}\right)$. Finally, using (6.1) again, we infer $\partial_{1} u \in \mathcal{C}_{T}\left(H_{t a n, \gamma}^{m-2}\right)$.

\subsection{Normal derivatives}

The last step is again by induction, as in [24], page 867, (ii). For convenience of the reader, we provide a brief sketch of the proof.

Suppose that for some fixed $k$, with $1 \leq k<[m / 2]$, it has already been shown that $Z^{\alpha} \partial_{1}^{h} u$ belongs to $C_{T}\left(L^{2}\right)$, for any $h$ and $\alpha$ such that $h=1, \cdots, k,|\alpha|+2 h \leq m$. From (6.1) it immediately follows that $Z^{\alpha} \partial_{1}^{k+1} u^{I} \in C_{T}\left(L^{2}\right)$. It rests to prove that $Z^{\alpha} \partial_{1}^{k+1} u^{I I} \in C_{T}\left(L^{2}\right)$.

We apply operator $Z^{\alpha} \partial_{1}^{k+1},|\alpha|+2 k=m-2$, to the part $I I$ of (1.1) and obtain an equation similar to (6.9) of the form

$$
\left(\tilde{\mathcal{L}}+\tilde{\mathcal{C}}_{k}\right) Z^{\alpha} \partial_{1}^{k+1} u^{I I}=\mathcal{G}_{k},
$$

where $\tilde{\mathcal{C}}_{k} \in \mathcal{C}_{T}\left(H_{*, \gamma}^{s-3}\right)$ is a suitable linear operator. The right-hand side $\mathcal{G}_{k}$ contains derivatives of $u$ of order $m$ (in $H_{*, \gamma}^{m}$, i.e. counting 1 for each tangential derivative and 2 for normal derivatives), but contains only normal derivatives that have already been estimated. We infer $\mathcal{G}_{k} \in L^{2}\left(Q_{T}\right)$. Again it is crucial that the boundary matrix 
of $\tilde{\mathcal{L}}$ vanishes at $\left\{x_{1}=0\right\}$. We infer that the solution $Z^{\alpha} \partial_{1}^{k+1} u^{I I}$ is in $C_{T}\left(L^{2}\right)$ for all $\alpha, k$ with $|\alpha|+2 k=m-2$. By repeating this procedure we obtain the result for any $k \leq[m / 2]$, hence $u \in \mathcal{C}_{T}\left(H_{*, \gamma}^{m}\right)$.

The apriori estimate (1.13) follows from (6.7) plus standard $L^{2}$ energy estimates for equations (6.9) and (6.11), and the direct estimate of the normal derivative of $u$ by tangential derivatives via (6.1). All products of functions are estimated in spaces $H_{*, \gamma}^{m}$ by the rules given in Theorem Appendix B.5 and Lemmata Appendix B.10 and Appendix B.11. We refer the reader to $[6,24,25]$ for similar details.

This concludes the proof of Theorem 1.2.

\section{Acknowledgement}

The work was supported by the national research project PRIN 2007 "Equations of Fluid Dynamics of Hyperbolic Type and Conservation Laws".

\section{Appendix A. Proof of Theorem 1.1}

Assume that all the hypotheses of Theorem 1.1 hold. Given the operator $L$ with matrix $A_{1}$ as in (2) we may associate some strictly dissipative boundary conditions, namely we may find a boundary matrix $M_{1}$, with ker $A_{1} \subseteq \operatorname{ker} M_{1}$, and there exists a constant $\epsilon>0$ such that

$$
-\left\langle A_{1}(x, t) w, w\right\rangle \geq \epsilon\left|w^{I}\right|^{2}-\frac{1}{\epsilon}\left|M_{1} w\right|^{2} \quad \forall w \in \mathbb{R}^{N},(x, t) \in \Sigma,
$$

where $\langle\cdot, \cdot\rangle$ denotes the scalar product in $\mathbb{R}^{N}$. Let us consider the initial-boundary value problem

$$
\begin{array}{ll}
L w=F & \text { in } Q_{T}, \\
M_{1} w=0 & \text { on } \Sigma_{T}, \\
w_{\mid t=0}=f & \text { in } \mathbb{R}_{+}^{n} .
\end{array}
$$

It is well known, see e.g. [4], that (A.2) admits a unique strong solution $w \in$ $C\left([0, T] ; L^{2}\left(\mathbb{R}_{+}^{n}\right)\right)$ with $w_{\mid \Sigma_{T}}^{I} \in L^{2}\left(\Sigma_{T}\right)$. Moreover, using (A.1) a standard argument gives the a priori estimate for $w_{\gamma}=e^{-\gamma t} w$

$$
\gamma\left\|w_{\gamma}\right\|_{L^{2}\left(Q_{t}\right)}^{2}+\left\|w_{\gamma}(t)\right\|_{L^{2}\left(\mathbb{R}_{+}^{n}\right)}^{2}+\left\|w_{\gamma \mid \Sigma_{t}}^{I}\right\|_{L^{2}\left(\Sigma_{t}\right)}^{2} \leq C\left(\|f\|_{L^{2}\left(\mathbb{R}_{+}^{n}\right)}^{2}+\frac{1}{\gamma}\left\|F_{\gamma}\right\|_{L^{2}\left(Q_{t}\right)}^{2}\right)
$$

for all $\gamma \geq \gamma_{0}$ and $0<t \leq T$, where $\gamma_{0}$ is taken sufficiently large.

For our subsequent use we need an estimate of $w_{\gamma \mid \Sigma_{T}}^{I}$ in $H_{\gamma}^{1}\left(\Sigma_{T}\right)$. Applying the operators $Z_{i}$ to (A.2) and taking account of (5.6), we infer that $Z w=\left(Z_{1} w, \ldots, Z_{n+1} w\right)$ (where $Z_{n+1}=\partial_{t}$ ) solves the problem

$$
\begin{array}{ll}
L Z_{i} w+\sum_{|\beta|=1} \Gamma_{\beta} Z^{\beta} w=\left(Z_{i}+\Psi\right) F+\Gamma_{0} w, & \text { in } Q_{T}, \\
M_{1} Z_{i} w=-\left(Z_{i} M_{1}\right) w, & \text { on } \Sigma_{T}, \\
Z_{i} w_{\mid t=0}=Z_{i} f, & \text { in } \mathbb{R}_{+}^{n} .
\end{array}
$$


An analysis of the commutator formula (5.6), similar to that of [18, Lemma C.1], yields $\Gamma_{\beta} \in L^{\infty}\left(Q_{T}\right), \Gamma_{0} \in L^{\infty}\left(Q_{T}\right), \Psi \in W^{1, \infty}\left(Q_{T}\right)$. Moreover, the assumptions on $F, f$ in Theorem 1.1 yield $\left(Z_{i}+\Psi\right) F \in L^{2}\left(Q_{T}\right), Z_{i} f \in L^{2}\left(\mathbb{R}_{+}^{n}\right)$, and therefore $Z_{i} w \in C\left([0, T] ; L^{2}\left(\mathbb{R}_{+}^{n}\right)\right)$ with $Z_{i} w_{\mid \Sigma_{T}}^{I} \in L^{2}\left(\Sigma_{T}\right)$. Using the same argument as for (A.3) gives

$$
\begin{aligned}
& \gamma\left\|(Z w)_{\gamma}\right\|_{L^{2}\left(Q_{t}\right)}^{2}+\left\|(Z w)_{\gamma}(t)\right\|_{L^{2}\left(\mathbb{R}_{+}^{n}\right)}^{2}+\left\|(Z w)_{\gamma \mid \Sigma_{t}}^{I}\right\|_{L^{2}\left(\Sigma_{t}\right)}^{2} \\
& \leq C\left(\|Z f\|_{L^{2}\left(\mathbb{R}_{+}^{n}\right)}^{2}+\left\|w_{\gamma \mid \Sigma_{t}}^{I}\right\|_{L^{2}\left(\Sigma_{t}\right)}^{2}+\frac{1}{\gamma}\left\|\left(\left(Z_{i}+\Psi\right) F+\Gamma_{0} w\right)_{\gamma}\right\|_{L^{2}\left(Q_{t}\right)}^{2}\right),
\end{aligned}
$$

which gives, combining with (A.3), the estimate

$$
\begin{aligned}
& \gamma\left\|w_{\gamma}\right\|_{H_{\text {tan, }}^{1}\left(Q_{t}\right)}^{2}+\left\||| w_{\gamma}(t)\right\|_{1, t a n, \gamma}^{2}+\left\|w_{\gamma \mid \Sigma_{t}}^{I}\right\|_{H_{\gamma}^{1}\left(\Sigma_{t}\right)}^{2} \\
& \leq C\left(\left.\|f\|\right|_{1, t a n, \gamma} ^{2}+\frac{1}{\gamma}\left\|F_{\gamma}\right\|_{H_{t a n, \gamma}^{1}\left(Q_{t}\right)}^{2}\right)
\end{aligned}
$$

for all $\gamma \geq \gamma_{0}$ and $0<t \leq T$, where $\gamma_{0}$ is taken sufficiently large.

Now we consider the initial-boundary value problem

$$
\begin{array}{ll}
L v=0 & \text { in } Q_{T}, \\
M v=G-M w & \text { on } \Sigma_{T}, \\
v_{\mid t=0}=0 & \text { in } \mathbb{R}_{+}^{n} .
\end{array}
$$

Since $(G-M w)_{\mid t=0}=G_{\mid t=0}-M f=0$ on $\mathbb{R}^{n-1}$, we may extend $(G-M w)_{\mid \Sigma_{T}}$ from $[0, T]$ to $]-\infty, T]$ by setting it equal to zero for all negative times and get a function in $H_{\gamma}^{1}\left(\omega_{T}\right)$. By assumption (4) there exists the solution of (A.5) $v \in L^{2}\left(Q_{T}\right)$ such that $v_{\mid \Sigma_{T}}^{I} \in L^{2}\left(\Sigma_{T}\right)$. Furthermore $v \in C\left([0, T] ; L^{2}\left(\mathbb{R}_{+}^{n}\right)\right)$, and it satisfies the a priori estimate

$$
\gamma\left\|v_{\gamma}\right\|_{L^{2}\left(Q_{t}\right)}^{2}+\left\|v_{\gamma}(t)\right\|_{L^{2}\left(\mathbb{R}_{+}^{n}\right)}^{2}+\left\|v_{\gamma \mid \Sigma_{t}}^{I}\right\|_{L^{2}\left(\Sigma_{t}\right)}^{2} \leq \frac{C}{\gamma^{2}}\left\|(G-M w)_{\gamma}\right\|_{H_{\gamma}^{1}\left(\Sigma_{t}\right)}^{2}
$$

for all $\gamma$ sufficiently large and $0<t \leq T$. It is clear that $u=v+w$ is a solution of (1.1)-(1.3) with the required properties; combining (A.3), (A.4), (A.6) gives (1.11). Finally, we observe that the uniqueness of the solution to (1.1)-(1.3) is a consequence of (4). The proof of Theorem 1.1 is complete.

\section{Appendix B. Properties of anisotropic Sobolev spaces}

Most of the theorems that we prove in this Appendix have already appeared in [18] in the $\gamma$-independent version. Here we prove the results with $\gamma$-dependent norms, and our main concern is to show that the a priori estimates of the previous sections do not explode but are uniformly controlled when $\gamma$ is taken sufficiently large.

In the sequel, we denote by $C_{(0)}^{\infty}\left(\mathbb{R}_{+}^{n}\right)$ the set of restrictions to $\mathbb{R}_{+}^{n}$ of functions of $C_{0}^{\infty}\left(\mathbb{R}^{n}\right)$. A similar definition is given for $C_{(0)}^{\infty}\left(Q_{T}\right)$. We also denote by $C_{B}^{0}\left(\mathbb{R}_{+}^{n}\right)$ the space of all bounded continuous functions over $\mathbb{R}_{+}^{n}$.

Theorem Appendix B.1. For all integers $m \geq 1$ and $\gamma \geq 1, C_{(0)}^{\infty}\left(\mathbb{R}_{+}^{n}\right)$ is dense in $H_{*, \gamma}^{m}\left(\mathbb{R}_{+}^{n}\right)$. 
44 A. Morando \&s P. Secchi

Proof. See [20].

Theorem Appendix B.2. Let $n \geq 2$ and $\gamma \geq 1$. For every integer $m \geq\left[\frac{n+1}{2}\right]+1$ the continuous imbedding $H_{*, \gamma}^{m}\left(\mathbb{R}_{+}^{n}\right) \hookrightarrow C_{B}^{0}\left(\mathbb{R}_{+}^{n}\right)$ holds. Moreover, there exists a constant $C$ such that for every $u \in H_{*, \gamma}^{m}\left(\mathbb{R}_{+}^{n}\right)$

$$
\left.\gamma^{m-(n+1) / 2}|| u\right|_{L^{\infty}\left(\mathbb{R}_{+}^{n}\right)} \leq\left. C|| u\right|_{H_{*, \gamma}^{m}\left(\mathbb{R}_{+}^{n}\right)} \quad \forall \gamma \geq 1 .
$$

Proof. As $C_{(0)}^{\infty}\left(\mathbb{R}_{+}^{n}\right)$ is dense in $H_{*, \gamma}^{m}\left(\mathbb{R}_{+}^{n}\right)$ by Theorem Appendix B.1, it is sufficient to prove (B.1) for $u \in C_{(0)}^{\infty}\left(\mathbb{R}_{+}^{n}\right)$. Given any $x_{1}$, let $\hat{u}$ denote the partial Fourier transform of $u$ defined by

$$
\hat{u}\left(x_{1}, \xi^{\prime}\right)=\int_{\mathbb{R}^{n-1}} e^{-i x^{\prime} \cdot \xi^{\prime}} u\left(x_{1}, x^{\prime}\right) d x^{\prime} .
$$

We have

$$
\begin{aligned}
& \left|u\left(x_{1}, x^{\prime}\right)\right|=(2 \pi)^{-n+1}\left|\int_{\mathbb{R}^{n-1}} e^{i \xi^{\prime} \cdot x^{\prime}} \lambda^{m-1, \gamma}\left(\xi^{\prime}\right) \hat{u}\left(x_{1}, \xi^{\prime}\right) \lambda^{-(m-1), \gamma}\left(\xi^{\prime}\right) d \xi^{\prime}\right| \\
& \leq(2 \pi)^{-(n-1) / 2} \|\left. u\left(x_{1}, \cdot\right)\right|_{H_{\gamma}^{m-1}\left(\mathbb{R}^{n-1}\right)}\left(\int_{\mathbb{R}^{n-1}} \lambda^{-2(m-1), \gamma}\left(\xi^{\prime}\right) d \xi^{\prime}\right)^{1 / 2} .
\end{aligned}
$$

We estimate

$$
\begin{aligned}
& \int_{\left\{\left|\xi^{\prime}\right| \leq A\right\}} \lambda^{-2(m-1), \gamma}\left(\xi^{\prime}\right) d \xi^{\prime} \leq C \frac{A^{n-1}}{\gamma^{2(m-1)}}, \\
& \int_{\left\{\left|\xi^{\prime}\right| \geq A\right\}} \lambda^{-2(m-1), \gamma}\left(\xi^{\prime}\right) d \xi^{\prime} \leq \frac{C}{A^{2 m-n-1}},
\end{aligned}
$$

(for the convergence of the second integral we use $2(m-1)>n-1)$ and the best $A=\gamma$ gives

$$
\int_{\mathbb{R}^{n-1}} \lambda^{-2(m-1), \gamma}\left(\xi^{\prime}\right) d \xi^{\prime} \leq C \gamma^{-(2 m-n-1)} .
$$

Substituting into (B.2) gives

$$
\gamma^{m-(n+1) / 2}\left\|u\left(x_{1}, \cdot\right)\right\|_{L^{\infty}\left(\mathbb{R}^{n-1}\right)} \leq C\left\|u\left(x_{1}, \cdot\right)\right\|_{H_{\gamma}^{m-1}\left(\mathbb{R}^{n-1}\right)} .
$$

On the other hand, we have $(\Re=$ real part $)$

$$
\begin{aligned}
& \left\|u\left(x_{1}, \cdot\right)\right\|_{H_{\gamma}^{m-1}\left(\mathbb{R}^{n-1}\right)}^{2}=(2 \pi)^{-n+1} \int_{\mathbb{R}^{n-1}}\left|\lambda^{m-1, \gamma}\left(\xi^{\prime}\right) \hat{u}\left(x_{1}, \xi^{\prime}\right)\right|^{2} d \xi^{\prime} \\
& =-(2 \pi)^{-n+1} \int_{\mathbb{R}^{n-1}} \lambda^{2(m-1), \gamma}\left(\xi^{\prime}\right)\left(\int_{x_{1}}^{\infty} \partial_{1}\left|\hat{u}\left(\xi_{1}, \xi^{\prime}\right)\right|^{2} d \xi_{1}\right) d \xi^{\prime} \\
& =-(2 \pi)^{-n+1} \int_{\mathbb{R}^{n-1}}^{\infty} \lambda^{2(m-1), \gamma}\left(\xi^{\prime}\right) 2 \Re\left(\int_{x_{1}}^{\infty} \hat{u}(\xi) \partial_{1} \overline{\hat{u}(\xi)} d \xi_{1}\right) d \xi^{\prime} \\
& =-2(2 \pi)^{-n+1} \Re \int_{x_{1}}^{+\infty} \int_{\mathbb{R}^{n-1}} \lambda^{m, \gamma}\left(\xi^{\prime}\right) \hat{u}(\xi) \times \lambda^{m-2, \gamma}\left(\xi^{\prime}\right) \partial_{1} \overline{\hat{u}(\xi)} d \xi \\
& \leq 2\|u\|_{H_{*, \gamma}^{m}\left(\mathbb{R}_{+}^{n}\right)}^{2}
\end{aligned}
$$

by application of the Cauchy-Schwarz inequality. Thus we have

$$
\left\|\left.u\left(x_{1}, \cdot\right)\right|_{H_{\gamma}^{m-1}\left(\mathbb{R}^{n-1}\right)} \leq \sqrt{2}\right\| u \|_{H_{*, \gamma}^{m}\left(\mathbb{R}_{+}^{n}\right)} .
$$


From (B.3) and (B.4) we obtain the thesis.

The following theorem provides some summability properties of anisotropic Sobolev functions with low order of regularity.

Theorem Appendix B.3. Let $\gamma \geq 1$. The following continuous imbeddings hold true:

a. If $n>5$ and $2 \leq m<\frac{n-1}{2}$, then

$$
H_{*, \gamma}^{m}\left(\mathbb{R}_{+}^{n}\right) \hookrightarrow L^{r}\left(\mathbb{R}_{+}^{n}\right), \quad \forall r \in\left[2, r^{*}\right], \quad \frac{1}{r^{*}}=\frac{1}{2}-\frac{m}{n+1} .
$$

There exists a constant $C$ such that for every $u \in H_{*, \gamma}^{m}\left(\mathbb{R}_{+}^{n}\right)$

$$
\gamma^{m-(n+1)(1 / 2-1 / r)}\left\|\left.u\right|_{L^{r}\left(\mathbb{R}_{+}^{n}\right)} \leq C\right\| u \|_{H_{*, \gamma}^{m}\left(\mathbb{R}_{+}^{n}\right)} \quad \forall \gamma \geq 1, r \in\left[2, r^{*}\right] .
$$

b. If $n \geq 5$ is odd, then

$$
H_{*, \gamma}^{(n-1) / 2}\left(\mathbb{R}_{+}^{n}\right) \hookrightarrow L^{r}\left(\mathbb{R}_{+}^{n}\right), \quad \forall r \in[2, n+1[.
$$

There exists a constant $C$ such that for every $u \in H_{*, \gamma}^{(n-1) / 2}\left(\mathbb{R}_{+}^{n}\right)$

$$
\gamma^{(n+1) / r-1}\|u\|_{L^{r}\left(\mathbb{R}_{+}^{n}\right)} \leq C|| u \|_{H_{*, \gamma}^{(n-1) / 2}\left(\mathbb{R}_{+}^{n}\right)} \quad \forall \gamma \geq 1, r \in[2, n+1[.
$$

c. If $n \geq 4$ is even, then

$$
H_{*, \gamma}^{n / 2}\left(\mathbb{R}_{+}^{n}\right) \hookrightarrow L^{r}\left(\mathbb{R}_{+}^{n}\right), \quad \forall r \in[2,2 n] .
$$

There exists a constant $C$ such that for every $u \in H_{*, \gamma}^{n / 2}\left(\mathbb{R}_{+}^{n}\right)$

$$
\begin{array}{r}
\gamma^{1 / 2 n}\|u\|_{L^{2 n}\left(\mathbb{R}_{+}^{n}\right)} \leq C\|u\|_{H_{*, \gamma}^{n / 2}\left(\mathbb{R}_{+}^{n}\right)}, \\
\gamma^{1 / 2+1 / n}\|u\|_{L^{n}\left(\mathbb{R}_{+}^{n}\right)} \leq C\|u\|_{H_{*, \gamma}^{n / 2}\left(\mathbb{R}_{+}^{n}\right)} \quad \forall \gamma \geq 1 .
\end{array}
$$

Proof. a. For $n>5$, let $m$ be a given integer such that $2 \leq m<\frac{n-1}{2}$. In order to prove the first imbedding (B.5), firstly we use the standard Sobolev imbedding

$$
H^{m-1}\left(\mathbb{R}^{n-1}\right) \hookrightarrow L^{q}\left(\mathbb{R}^{n-1}\right),
$$

where $\frac{1}{q}=\frac{1}{2}-\frac{m-1}{n-1}>0$, to find the inequality

$$
\left\|u\left(x_{1}, \cdot\right)\right\|_{L^{q}\left(\mathbb{R}^{n-1}\right)} \leq C\left\|u\left(x_{1}, \cdot\right)\right\|_{H^{m-1}\left(\mathbb{R}^{n-1}\right)} \leq C\left\|u\left(x_{1}, \cdot\right)\right\|_{H_{\gamma}^{m-1}\left(\mathbb{R}^{n-1}\right)} \quad \forall \gamma \geq 1 .
$$

Then, using (B.4) gives

$$
\|u\|_{L^{\infty}\left(0,+\infty ; L^{q}\left(\mathbb{R}^{n-1}\right)\right)} \leq C|| u \|_{H_{*, \gamma}^{m}\left(\mathbb{R}_{+}^{n}\right)} .
$$

Similarly, from the imbedding $H^{m}\left(\mathbb{R}^{n-1}\right) \hookrightarrow L^{p}\left(\mathbb{R}^{n-1}\right)$, with $\frac{1}{p}=\frac{1}{2}-\frac{m}{n-1}>0$, we derive

$$
\|u\|_{L^{2}\left(0,+\infty ; L^{p}\left(\mathbb{R}^{n-1}\right)\right)}^{2} \leq C \int_{0}^{+\infty}\left\|u\left(x_{1}, \cdot\right)\right\|_{H_{\gamma}^{m}\left(\mathbb{R}^{n-1}\right)}^{2} d x_{1} \leq C\|u\|_{H_{*, \gamma}^{m}\left(\mathbb{R}_{+}^{n}\right)}^{2},
$$


for a suitable $C>0$ independent of $u$. Let $\theta$ be arbitrarily fixed in ]0,1[. For $\frac{1}{r}=\frac{\theta}{q}+\frac{1-\theta}{p}$, interpolating between $L^{q}\left(\mathbb{R}^{n-1}\right)$ and $L^{p}\left(\mathbb{R}^{n-1}\right)$ and Fubini's theorem gives

$$
\begin{aligned}
& \|u\|_{L^{r}\left(\mathbb{R}_{+}^{n}\right)}^{r}=\int_{0}^{+\infty}\left\|u\left(x_{1}, \cdot\right)\right\|_{L^{r}\left(\mathbb{R}^{n-1}\right)}^{r} d x_{1} \\
& \leq \int_{0}^{+\infty}\left\|u\left(x_{1}, \cdot\right)\right\|_{L^{q}\left(\mathbb{R}^{n-1}\right)}^{r \theta}\left\|u\left(x_{1}, \cdot\right)\right\|_{L^{p}\left(\mathbb{R}^{n-1}\right)}^{r(1-\theta)} d x_{1} \\
& \leq\|u\|_{L^{\infty}\left(0,+\infty ; L^{q}\left(\mathbb{R}^{n-1}\right)\right)}^{r \theta} \int_{0}^{+\infty}\left\|u\left(x_{1}, \cdot\right)\right\|_{L^{p}\left(\mathbb{R}^{n-1}\right)}^{r(1-\theta)} d x_{1} .
\end{aligned}
$$

Setting $r(1-\theta)=2$, from $\frac{1}{r}=\frac{\theta}{q}+\frac{1-\theta}{p}$ we compute for $\theta$ and $r$ the values

$$
\theta=\theta^{*}:=\frac{\frac{1}{2}-\frac{1}{p}}{\frac{1}{2}-\frac{1}{p}+\frac{1}{q}}=\frac{2 m}{n+1}, \quad \frac{1}{r}=\frac{1}{r^{*}}:=\frac{1}{2}-\frac{m}{n+1} .
$$

Setting $r=r^{*}$ and $\theta=\theta^{*}$ in (B.15) and using estimates (B.13) and (B.14), we get

$$
\|u\|_{L^{r^{*}\left(\mathbb{R}_{+}^{n}\right)}} \leq C\|u\|_{H_{*, \gamma}^{m}\left(\mathbb{R}_{+}^{n}\right)},
$$

which proves the imbedding $H_{*, \gamma}^{m}\left(\mathbb{R}_{+}^{n}\right) \hookrightarrow L^{r^{*}}\left(\mathbb{R}_{+}^{n}\right)$. The imbedding $H_{*, \gamma}^{m}\left(\mathbb{R}_{+}^{n}\right) \hookrightarrow$ $L^{r}\left(\mathbb{R}_{+}^{n}\right)$ for all $r \in\left[2, r^{*}\right]$ immediately follows from the interpolation between $L^{2}\left(\mathbb{R}_{+}^{n}\right)$ and $L^{r^{*}}\left(\mathbb{R}_{+}^{n}\right)$. This ends the proof of (B.5). Finally, interpolating between (B.16) and the inequality $\gamma^{m}\|u\|_{L^{2}\left(\mathbb{R}_{+}^{n}\right)} \leq C\|u\|_{H_{*, \gamma}^{m}\left(\mathbb{R}_{+}^{n}\right)}$ yields (B.6).

b. Assume now that $n$ is odd and $\geq 5$. Here we have the difficulty of the limiting case of Sobolev imbedding theorem that $H^{(n-1) / 2}\left(\mathbb{R}^{n-1}\right)$ is not imbedded into $L^{\infty}\left(\mathbb{R}^{n-1}\right)$; in order to solve it we argue as follows.

Given any two positive real numbers $h_{1}$ and $h_{2}$ such that $h_{1}<\frac{n-1}{2}<h_{2}$, the imbedding $A: H_{\gamma}^{h_{1}}\left(\mathbb{R}^{n-1}\right) \rightarrow L^{2}\left(\mathbb{R}^{n-1}\right)$ is a bounded linear operator with norm $M_{0} \leq C \gamma^{-h_{1}}$, as follows from (2.3). From (B.3), $A$ is also a bounded linear operator from $H_{\gamma}^{h_{2}}\left(\mathbb{R}^{n-1}\right)$ into $L^{\infty}\left(\mathbb{R}^{n-1}\right)$, with norm $M_{1} \leq C \gamma^{-\left(h_{2}-(n-1) / 2\right)}$. By interpolation, see [5], it follows that $A$ is a bounded linear operator from $\left(H_{\gamma}^{h_{1}}, H_{\gamma}^{h_{2}}\right)_{\theta, 2}$ into $\left(L^{2}, L^{\infty}\right)_{\theta, 2}$ as well, for any $0<\theta<1$, with norm

$$
M_{\theta} \leq M_{0}^{1-\theta} M_{1}^{\theta} \leq C \gamma^{-\left[h_{1}(1-\theta)+\left(h_{2}-(n-1) / 2\right) \theta\right]} .
$$

We have $\left(H_{\gamma}^{h_{1}}, H_{\gamma}^{h_{2}}\right)_{\theta, 2}=H_{\gamma}^{h_{1}(1-\theta)+h_{2} \theta}$ and $\left(L^{2}, L^{\infty}\right)_{\theta, 2}=L^{a(\theta), 2}$ where $a(\theta)=$ $2 /(1-\theta)$; moreover $L^{a(\theta), 2} \subset L^{a(\theta)}$ because $a(\theta)>2$. Now, given any $p$ such that $2<p<\infty$, we take $\theta=1-2 / p$ so that $a(\theta)=p$, and consequently choose $h_{1}<\frac{n-1}{2}<h_{2}$ such that $h_{1}(1-\theta)+h_{2} \theta=\frac{n-1}{2}$. It follows that $A$ is a bounded linear operator from $H_{\gamma}^{(n-1) / 2}\left(\mathbb{R}^{n-1}\right)$ into $L^{p}\left(\mathbb{R}^{n-1}\right)$, and from (B.17) we get the estimate

$$
\gamma^{(n-1) / p}\left\|u\left(x_{1}, \cdot\right)\right\|_{L^{p}\left(\mathbb{R}^{n-1}\right)} \leq C\left\|u\left(x_{1}, \cdot\right)\right\|_{H_{\gamma}^{(n-1) / 2}\left(\mathbb{R}^{n-1}\right)}, \quad 2<p<\infty .
$$

From (B.18) and integration w.r.t. $x_{1}$ it readily follows

$$
\gamma^{(n-1) / p}|| u\left\|_{L^{2}\left(0,+\infty ; L^{p}\left(\mathbb{R}^{n-1}\right)\right)} \leq C|| u\right\|_{H_{*, \gamma}^{(n-1) / 2}\left(\mathbb{R}_{+}^{n}\right)}, \quad 2 \leq p<\infty .
$$


From the inequality (B.13) with $m=\frac{n-1}{2}$ and $q=n-1$ (recall that (B.13) follows from (B.12) that is true as long as $m-1<\frac{n-1}{2}$ ) we get

$$
\|u\|_{L^{\infty}\left(0,+\infty ; L^{n-1}\left(\mathbb{R}^{n-1}\right)\right)} \leq C|| u \|_{H_{*, \gamma}^{(n-1) / 2}\left(\mathbb{R}_{+}^{n}\right)} .
$$

Interpolating between (B.19) and (B.20) we derive

$$
\gamma^{(n+1) / r-1}|| u\left\|_{L^{r}\left(\mathbb{R}_{+}^{n}\right)} \leq C\right\| u \|_{H_{*, \gamma}^{(n-1) / 2}\left(\mathbb{R}_{+}^{n}\right)},
$$

where $r=2(n-1)\left(\frac{1}{2}-\frac{1}{p}+\frac{1}{n-1}\right)$. Then the continuous imbedding (B.7) follows by noticing that the map $p \mapsto r(p)=2(n-1)\left(\frac{1}{2}-\frac{1}{p}+\frac{1}{n-1}\right)$ is increasing and continuous over $[2,+\infty[$ and $r(p) \nearrow n+1$ as $p \nearrow+\infty$. From (B.21) we have (B.8).

c. To conclude, we prove the continuous imbedding (B.9) and (B.10). Thus, we assume that $n \geq 4$ is even. Again, by (B.13) for $m=\frac{n}{2}$ we derive that

$$
\|u\|_{L^{\infty}\left(0,+\infty ; L^{\left.2(n-1)\left(\mathbb{R}^{n-1}\right)\right)}\right.} \leq C\|u\|_{H_{*, \gamma}^{n / 2}\left(\mathbb{R}_{+}^{n}\right)} .
$$

Moreover, applying (B.3) with $m=n / 2+1>(n+1) / 2$ gives

$$
\gamma^{\frac{1}{2}}\|u\|_{L^{2}\left(0,+\infty ; L^{\infty}\left(\mathbb{R}^{n-1}\right)\right)} \leq C\|u\|_{H_{*, \gamma}^{n / 2}\left(\mathbb{R}_{+}^{n}\right)} .
$$

For all $r>q$ we find

$$
\left\|u\left(x_{1}, \cdot\right)\right\|_{L^{r}\left(\mathbb{R}^{n-1}\right)}^{r} \leq\left\|u\left(x_{1}, \cdot\right)\right\|_{L^{\infty}\left(\mathbb{R}^{n-1}\right)}^{r-q}\left\|u\left(x_{1}, \cdot\right)\right\|_{L^{q}\left(\mathbb{R}^{n-1}\right)}^{q},
$$

which yields

$$
\begin{aligned}
& \|u\|_{L^{r}\left(\mathbb{R}_{+}^{n}\right)}^{r}=\int_{0}^{+\infty}\left\|u\left(x_{1}, \cdot\right)\right\|_{L^{r}\left(\mathbb{R}^{n-1}\right)}^{r} d x_{1} \\
& \leq\|u\|_{L^{\infty}\left(0,+\infty ; L^{q}\left(\mathbb{R}^{n-1}\right)\right)}^{q} \int_{0}^{+\infty}\left\|u\left(x_{1}, \cdot\right)\right\|_{L^{\infty}\left(\mathbb{R}^{n-1}\right)}^{r-q} d x_{1} .
\end{aligned}
$$

Setting now $r=q+2=2 n$ and using (B.22), (B.23), we derive the continuous imbedding $H_{*}^{\frac{n}{2}}\left(\mathbb{R}_{+}^{n}\right) \hookrightarrow L^{2 n}\left(\mathbb{R}_{+}^{n}\right)$ with (B.10). Interpolating between $L^{2}\left(\mathbb{R}_{+}^{n}\right)$ and $L^{2 n}\left(\mathbb{R}_{+}^{n}\right)$ gives the continuous imbeddings in (B.9) and (B.11).

Corollary Appendix B.4. Let $n \geq 4$ and let $2^{*}$ be defined by $1 / 2^{*}=1 / 2-1 / n$. There exists a constant $C$ independent of $\gamma$ such that

$$
\gamma^{1-1 / n}\|u\|_{L^{2^{*}}\left(\mathbb{R}_{+}^{n}\right)} \leq C\|u\|_{H_{*, \gamma}^{2}\left(\mathbb{R}_{+}^{n}\right)}, \quad \forall \gamma \geq 1,
$$

for any $u \in H_{*, \gamma}^{2}\left(\mathbb{R}_{+}^{n}\right)$.

Proof. The proof follows from (B.11) if $n=4$, (B.8) with $r=2^{*}$ if $n=5$, and from (B.6) with $r=2^{*}$ if $n>5$.

Observe that the standard Sobolev imbedding yields $\|u\|_{L^{2^{*}}\left(\mathbb{R}_{+}^{n}\right)} \leq$ $C\|u\|_{H^{1}\left(\mathbb{R}_{+}^{n}\right)} \leq C\|u\|_{H_{*, \gamma}^{2}\left(\mathbb{R}_{+}^{n}\right)}$. Thus (B.24) improves the dependence on $\gamma$.

The next theorem deals with the product of two anisotropic Sobolev functions, one of which may have a low order of regularity. 
Theorem Appendix B.5. Let $m \geq 1$ be an integer and $s=\max \left\{m,\left[\frac{n+1}{2}\right]+2\right\}$. For any $u \in H_{*, \gamma}^{m}\left(\mathbb{R}_{+}^{n}\right)$ and $v \in H_{*, \gamma}^{s}\left(\mathbb{R}_{+}^{n}\right)$ one has uv $\in H_{*, \gamma}^{m}\left(\mathbb{R}_{+}^{n}\right)$. Moreover, there exists a constant $C$ independent of $\gamma$ such that

$$
\gamma^{s-(n+1) / 2}\|u v\|_{H_{*, \gamma}^{m}\left(\mathbb{R}_{+}^{n}\right)} \leq C\|u\|_{H_{*, \gamma}^{m}\left(\mathbb{R}_{+}^{n}\right)}\|v\|_{H_{*, \gamma}^{s}\left(\mathbb{R}_{+}^{n}\right)}, \quad \forall \gamma \geq 1 .
$$

Proof. Let us assume first that $1 \leq m<s=\left[\frac{n+1}{2}\right]+2$. For $m=1$ the result is true as a consequence of the imbedding Theorem Appendix B.2; indeed for $u \in H_{*, \gamma}^{1}\left(\mathbb{R}_{+}^{n}\right)$ and $v \in H_{*, \gamma}^{s}\left(\mathbb{R}_{+}^{n}\right)$ we have

$$
\begin{aligned}
& \gamma^{s-(n+1) / 2} \gamma\|u v\|_{L^{2}\left(\mathbb{R}_{+}^{n}\right)} \leq \gamma^{s-(n+1) / 2+1}\|u\|_{L^{2}\left(\mathbb{R}_{+}^{n}\right)}\|v\|_{L^{\infty}\left(\mathbb{R}_{+}^{n}\right)} \\
& \leq C \gamma^{2}\|u\|_{L^{2}\left(\mathbb{R}_{+}^{n}\right)}\|v\|_{H_{*, \gamma}^{s-1}\left(\mathbb{R}_{+}^{n}\right)} \leq C\|u\|_{H_{*, \gamma}^{1}\left(\mathbb{R}_{+}^{n}\right)}\|v\|_{H_{*, \gamma}^{s}\left(\mathbb{R}_{+}^{n}\right)},
\end{aligned}
$$

and similarly

$$
\begin{aligned}
& \gamma^{s-(n+1) / 2}\left\|Z_{i}(u v)\right\|_{L^{2}\left(\mathbb{R}_{+}^{n}\right)} \\
& \leq C \gamma\left\|Z_{i} u\right\|_{L^{2}\left(\mathbb{R}_{+}^{n}\right)}\|v\|_{H_{*, \gamma}^{s-1}\left(\mathbb{R}_{+}^{n}\right)}+C \gamma\|u\|_{L^{2}\left(\mathbb{R}_{+}^{n}\right)}\left\|Z_{i} v\right\|_{H_{*, \gamma}^{s-1}\left(\mathbb{R}_{+}^{n}\right)} \\
& \leq C\|u\|_{H_{*, \gamma}^{1}\left(\mathbb{R}_{+}^{n}\right)}\|v\|_{H_{*, \gamma}^{s}\left(\mathbb{R}_{+}^{n}\right)} .
\end{aligned}
$$

For $2 \leq m \leq s-1$, assume the result has been already proven up to order $m-1$. By hypothesis, if $u \in H_{*, \gamma}^{m}\left(\mathbb{R}_{+}^{n}\right)$ and $v \in H_{*, \gamma}^{s}\left(\mathbb{R}_{+}^{n}\right)$, we know that $u v \in H_{*, \gamma}^{m-1}\left(\mathbb{R}_{+}^{n}\right)$ with

$$
\gamma^{s-(n+1) / 2}\|u v\|_{H_{*, \gamma}^{m-1}\left(\mathbb{R}_{+}^{n}\right)} \leq C\|u\|_{H_{*, \gamma}^{m-1}\left(\mathbb{R}_{+}^{n}\right)}\|v\|_{H_{*, \gamma}^{s}\left(\mathbb{R}_{+}^{n}\right)}, \quad \forall \gamma \geq 1 .
$$

It remains to prove that $Z^{\alpha} \partial_{1}^{k}(u v) \in L^{2}\left(\mathbb{R}_{+}^{n}\right)$, when $|\alpha|+2 k=m$, with a suitable a priori estimate. By Leibniz's formula, we compute

$$
\left\|Z^{\alpha} \partial_{1}^{k}(u v)\right\|_{L^{2}\left(\mathbb{R}_{+}^{n}\right)} \leq C \sum_{(\beta, h),(\gamma, l) \in I(\alpha, k)}\left\|Z^{\beta} \partial_{1}^{h} u Z^{\gamma} \partial_{1}^{l} v\right\|_{L^{2}\left(\mathbb{R}_{+}^{n}\right)},
$$

where $I(\alpha, k):=\{(\beta, h),(\gamma, l): \beta+\gamma=\alpha, h+l=k\}$. For later use notice that $(m-|\beta|-2 h)+(s-|\gamma|-2 l)=s$. Let us first assume $n \geq 4$. We split $I(\alpha, k)$ as $I(\alpha, k)=I_{1}(\alpha, k) \cup I_{2}(\alpha, k) \cup I_{3}(\alpha, k) \cup I_{4}(\alpha, k) \cup I_{5}(\alpha, k)$, where

$$
\begin{aligned}
& I_{1}(\alpha, k)::=\left\{(\beta, h),(\gamma, l) \in I(\alpha, k): 2 \leq m-|\beta|-2 h<\frac{n-1}{2}\right. \\
&\text { and } \left.2 \leq s-|\gamma|-2 l<\frac{n-1}{2}\right\} ; \\
& I_{2}(\alpha, k)::=\{(\beta, h),(\gamma, l) \in I(\alpha, k): m-|\beta|-2 h \leq 1\} ; \\
& I_{3}(\alpha, k):=\{(\beta, h),(\gamma, l) \in I(\alpha, k): s-|\gamma|-2 l \leq 1\} ; \\
& I_{4}(\alpha, k):=\left\{(\beta, h),(\gamma, l) \in I(\alpha, k): m-|\beta|-2 h \geq \frac{n-1}{2}\right\} ; \\
& I_{5}(\alpha, k):=\left\{(\beta, h),(\gamma, l) \in I(\alpha, k): s-|\gamma|-2 l \geq \frac{n-1}{2}\right\}
\end{aligned}
$$


(we remark that $I_{1}(\alpha, k)=\emptyset$, as long as $\left.n \leq 5\right)$. According to the splitting above, we decompose the sum in the right-hand side of (B.27) as:

$$
\sum_{(\beta, h),(\gamma, l) \in I(\alpha, k)}\left\|Z^{\beta} \partial_{1}^{h} u Z^{\gamma} \partial_{1}^{l} v\right\|_{L^{2}\left(\mathbb{R}_{+}^{n}\right)}=K_{1}+K_{2}+K_{3}+K_{4}+K_{5},
$$

and we estimate separately each term, where for $i=1, \ldots, 5$,

$$
K_{i}:=\sum_{(\beta, h),(\gamma, l) \in I_{i}(\alpha, k)}\left\|Z^{\beta} \partial_{1}^{h} u Z^{\gamma} \partial_{1}^{l} v\right\|_{L^{2}\left(\mathbb{R}_{+}^{n}\right)} .
$$

We consider $K_{1}$ (from the above remark $n \geq 6$ ). From Theorem Appendix B.3 a., we get for all $(\beta, h),(\gamma, l) \in I_{1}(\alpha, k)$

$$
\begin{aligned}
& Z^{\beta} \partial_{1}^{h} u \in H_{*, \gamma}^{m-|\beta|-2 h}\left(\mathbb{R}_{+}^{n}\right) \hookrightarrow L^{p}\left(\mathbb{R}_{+}^{n}\right), \quad \frac{1}{p}=\frac{1}{2}-\frac{m-|\beta|-2 h}{n+1} ; \\
& Z^{\gamma} \partial_{1}^{l} v \in H_{*, \gamma}^{s-|\gamma|-2 l}\left(\mathbb{R}_{+}^{n}\right) \hookrightarrow L^{q}\left(\mathbb{R}_{+}^{n}\right), \quad \frac{1}{q}=\frac{1}{2}-\frac{s-|\gamma|-2 l}{n+1} .
\end{aligned}
$$

We notice that $\frac{1}{p}+\frac{1}{q}=: \frac{1}{r}<\frac{1}{2}$. Let us denote $\theta_{1}:=\frac{p-r}{p-2}$ and $\theta_{2}=\frac{q-r}{q-2}$. From Theorem Appendix B.3 a., we get

$$
\begin{aligned}
& \gamma^{s-(n+1) / 2}\left\|Z^{\beta} \partial_{1}^{h} u Z^{\gamma} \partial_{1}^{l} v\right\|_{L^{2}\left(\mathbb{R}_{+}^{n}\right)} \leq \gamma^{s-(n+1) / 2}\left\|Z^{\beta} \partial_{1}^{h} u\right\|_{L^{\frac{2 p}{r}}\left(\mathbb{R}_{+}^{n}\right)}\left\|Z^{\gamma} \partial_{1}^{l} v\right\|_{L^{\frac{2 q}{r}}\left(\mathbb{R}_{+}^{n}\right)} \\
& \leq \gamma^{-(n+1) / 2}\left(\gamma^{m-|\beta|-2 h}\left\|Z^{\beta} \partial_{1}^{h} u\right\|_{L^{2}\left(\mathbb{R}_{+}^{n}\right)}\right)^{1-\theta_{1}}\left(\gamma^{m-|\beta|-2 h}|| Z^{\beta} \partial_{1}^{h} u \|_{L^{p}\left(\mathbb{R}_{+}^{n}\right)}\right)^{\theta_{1}} \times \\
& \times\left(\gamma^{s-|\gamma|-2 l}\left\|Z^{\gamma} \partial_{1}^{l} v\right\|_{L^{2}\left(\mathbb{R}_{+}^{n}\right)}\right)^{1-\theta_{2}}\left(\gamma^{s-|\gamma|-2 l}|| Z^{\gamma} \partial_{1}^{l} v \|_{L^{q}\left(\mathbb{R}_{+}^{n}\right)}\right)^{\theta_{2}} \\
& \leq C \gamma^{-(n+1) / 2} \gamma^{(m-|\beta|-2 h) \theta_{1}}\left\|\left.Z^{\beta} \partial_{1}^{h} u\right|_{H_{*, \gamma}^{m-|\beta|-2 h}\left(\mathbb{R}_{+}^{n}\right)} \gamma^{(s-|\gamma|-2 l) \theta_{2}}\right\| Z^{\gamma} \partial_{1}^{l} v \|_{H_{*, \gamma}^{s-|\gamma|-2 l}\left(\mathbb{R}_{+}^{n}\right)} \\
& \leq C \gamma^{-(n+1) / 2} \gamma^{(m-|\beta|-2 h) \theta_{1}} \gamma^{(s-|\gamma|-2 l) \theta_{2}}\|u\|_{H_{*, \gamma}^{m}\left(\mathbb{R}_{+}^{n}\right)}\|v\|_{H_{*, \gamma}^{s}\left(\mathbb{R}_{+}^{n}\right)} \\
& =C\|u\|_{H_{*, \gamma}^{m}\left(\mathbb{R}_{+}^{n}\right)}\|v\|_{H_{*, \gamma}^{s}\left(\mathbb{R}_{+}^{n}\right)} \text {. }
\end{aligned}
$$

Let us consider now $K_{2}$. For all $(\beta, h),(\gamma, l) \in I_{2}(\alpha, k)$, one has $|\beta|+2 h \geq m-1$ and $|\gamma|+2 l=m-(|\beta|+2 h) \leq 1$. Then, $Z^{\gamma} \partial_{1}^{l} v \in H_{*, \gamma}^{s-1}\left(\mathbb{R}_{+}^{n}\right) \hookrightarrow L^{\infty}\left(\mathbb{R}_{+}^{n}\right)$ by the imbedding Theorem Appendix B.2. One immediately derives

$$
\begin{aligned}
& \gamma^{s-(n+1) / 2}\left\|Z^{\beta} \partial_{1}^{h} u Z^{\gamma} \partial_{1}^{l} v\right\|_{L^{2}\left(\mathbb{R}_{+}^{n}\right)} \\
& \leq \gamma^{m-|\beta|-2 h}\left\|Z^{\beta} \partial_{1}^{h} u\right\|_{L^{2}\left(\mathbb{R}_{+}^{n}\right)} \gamma^{(s-|\gamma|-2 l)-(n+1) / 2}\left\|Z^{\gamma} \partial_{1}^{l} v\right\|_{L^{\infty}\left(\mathbb{R}_{+}^{n}\right)} \\
& \leq C\|u\|_{H_{*, \gamma}^{m}\left(\mathbb{R}_{+}^{n}\right)}\|v\|_{H_{*, \gamma}^{s}\left(\mathbb{R}_{+}^{n}\right)} .
\end{aligned}
$$

Let us estimate $K_{3}$. Firstly, we observe that for $2 \leq m<s-1, I_{3}(\alpha, k)$ is empty, because otherwise $(\gamma, l) \in I_{3}(\alpha, k)$ would satisfy both $|\gamma|+2 l \geq s-1$ and $|\gamma|+2 l \leq$ $|\alpha|+2 k=m$. For $m=s-1$, one computes that all $(\beta, h),(\gamma, l) \in I_{3}(\alpha, k)$ satisfy $|\gamma|+2 l=m$ (thus $(\gamma, l)=(\alpha, k))$ and $|\beta|+2 h=m-(|\gamma|+2 l)=0$ (thus $(\beta, h)=$ $(0,0))$. Again by Theorem Appendix B.2 (applied to $\left.H_{*, \gamma}^{m}\left(\mathbb{R}_{+}^{n}\right)=H_{*, \gamma}^{s-1}\left(\mathbb{R}_{+}^{n}\right)\right)$, this yields

$$
\begin{aligned}
& \gamma^{s-(n+1) / 2}\left\|Z^{\beta} \partial_{1}^{h} u Z^{\gamma} \partial_{1}^{l} v\right\|_{L^{2}\left(\mathbb{R}_{+}^{n}\right)}=\gamma^{s-(n+1) / 2}\left\|u Z^{\alpha} \partial_{1}^{k} v\right\|_{L^{2}\left(\mathbb{R}_{+}^{n}\right)} \\
& \leq \gamma^{s-1-(n+1) / 2}\|u\|_{L^{\infty}\left(\mathbb{R}_{+}^{n}\right)} \gamma\left\|Z^{\alpha} \partial_{1}^{k} v\right\|_{L^{2}\left(\mathbb{R}_{+}^{n}\right)} \leq C\|u\|_{H_{*, \gamma}^{m}\left(\mathbb{R}_{+}^{n}\right)}\|v\|_{H_{*, \gamma}^{s}\left(\mathbb{R}_{+}^{n}\right)} .
\end{aligned}
$$

Let us consider now the term $K_{4}$. We divide the proof in several steps.

i) First, we assume that $n \geq 4$ is even. Setting $n=2 k$ ( $k$ integer $\geq 2$ ), we compute 
that $\frac{n-1}{2}=k-\frac{1}{2}$ and $\left[\frac{n+1}{2}\right]+1=k+1$. Since each $(\beta, h) \in I_{4}(\alpha, k)$ satisfies $m-|\beta|-2 h \geq \frac{n-1}{2}$ and $m \leq\left[\frac{n+1}{2}\right]+1$, we deduce $k-\frac{1}{2} \leq m \leq k+1$, hence $k \leq m \leq k+1$.

i.1) For $m=k$, inequality $m-|\beta|-2 h \geq \frac{n-1}{2}$ implies that $|\beta|+2 h=0$ and $|\gamma|+2 l=m-|\beta|-2 h=k$. Hence, by Theorem Appendix B.3 c., we obtain

$$
Z^{\beta} \partial_{1}^{h} u=u \in H_{*, \gamma}^{m}\left(\mathbb{R}_{+}^{n}\right)=H_{*, \gamma}^{k}\left(\mathbb{R}_{+}^{n}\right)=H_{*, \gamma}^{\frac{n}{2}}\left(\mathbb{R}_{+}^{n}\right) \hookrightarrow L^{n}\left(\mathbb{R}_{+}^{n}\right)
$$

with estimate (B.11). On the other hand, since $s-k=\left[\frac{n+1}{2}\right]+2-k=2$, Corollary Appendix B.4 gives

$$
Z^{\gamma} \partial_{1}^{l} v \in H_{*, \gamma}^{s-k}\left(\mathbb{R}_{+}^{n}\right)=H_{*, \gamma}^{2}\left(\mathbb{R}_{+}^{n}\right) \hookrightarrow L^{2^{*}}\left(\mathbb{R}_{+}^{n}\right), \quad \frac{1}{2^{*}}=\frac{1}{2}-\frac{1}{n},
$$

with the estimate (B.24). Hence, we obtain

$$
\begin{aligned}
& \gamma^{s-(n+1) / 2}\left\|Z^{\beta} \partial_{1}^{h} u Z^{\gamma} \partial_{1}^{l} v\right\|_{L^{2}\left(\mathbb{R}_{+}^{n}\right)}=\gamma^{3 / 2}\left\|u Z^{\gamma} \partial_{1}^{l} v\right\|_{L^{2}\left(\mathbb{R}_{+}^{n}\right)} \\
& \leq \gamma^{1 / 2+1 / n}\|u\|_{L^{n}\left(\mathbb{R}_{+}^{n}\right)} \gamma^{1-1 / n}\left\|Z^{\gamma} \partial_{1}^{l} v\right\|_{L^{2^{*}}\left(\mathbb{R}_{+}^{n}\right)} \leq C\|u\|_{H_{*, \gamma}^{m}\left(\mathbb{R}_{+}^{n}\right)}\|v\|_{H_{*, \gamma}^{s}\left(\mathbb{R}_{+}^{n}\right)} .
\end{aligned}
$$

i.2) For $m=k+1$, we find that for $(\beta, h) \in I_{4}(\alpha, k), m-|\beta|-2 h=k+1-|\beta|-2 h \geq$ $k-\frac{1}{2}$ implies $|\beta|+2 h \leq 1$. We have to consider two cases.

i.2.1) For $|\beta|+2 h=0$, then $|\gamma|+2 l=m=k+1$; hence by Theorem Appendix B.2 we get $Z^{\beta} \partial_{1}^{h} u=u \in H_{*, \gamma}^{m}\left(\mathbb{R}_{+}^{n}\right)=H_{*, \gamma}^{\left[\frac{n+1}{2}\right]+1}\left(\mathbb{R}_{+}^{n}\right) \hookrightarrow L^{\infty}\left(\mathbb{R}_{+}^{n}\right)$, then

$$
\begin{aligned}
& \gamma^{s-(n+1) / 2}\left\|Z^{\beta} \partial_{1}^{h} u Z^{\gamma} \partial_{1}^{l} v\right\|_{L^{2}\left(\mathbb{R}_{+}^{n}\right)} \leq \gamma^{s-(n+1) / 2}\|u\|_{L^{\infty}\left(\mathbb{R}_{+}^{n}\right)}\left\|Z^{\gamma} \partial_{1}^{l} v\right\|_{L^{2}\left(\mathbb{R}_{+}^{n}\right)} \\
& \leq C \gamma^{s-m}\|u\|_{H_{*, \gamma}^{m}\left(\mathbb{R}_{+}^{n}\right)}\left\|Z^{\gamma} \partial_{1}^{l} v\right\|_{L^{2}\left(\mathbb{R}_{+}^{n}\right)} \leq C\|u\|_{H_{*, \gamma}^{m}\left(\mathbb{R}_{+}^{n}\right)}\|v\|_{H_{*, \gamma}^{s}\left(\mathbb{R}_{+}^{n}\right)} .
\end{aligned}
$$

i.2.2) For $|\beta|+2 h=1$ we have $|\gamma|+2 l=k$; then, as in step i.1), we find

$$
\begin{gathered}
Z^{\beta} \partial_{1}^{h} u \in H_{*, \gamma}^{k}\left(\mathbb{R}_{+}^{n}\right)=H_{*, \gamma}^{\frac{n}{2}}\left(\mathbb{R}_{+}^{n}\right) \hookrightarrow L^{n}\left(\mathbb{R}_{+}^{n}\right), \\
Z^{\gamma} \partial_{1}^{l} v \in H_{*, \gamma}^{s-k}\left(\mathbb{R}_{+}^{n}\right)=H_{*, \gamma}^{2}\left(\mathbb{R}_{+}^{n}\right) \hookrightarrow L^{2^{*}}\left(\mathbb{R}_{+}^{n}\right) .
\end{gathered}
$$

and we conclude as for i.1).

ii) Assume now that $n \geq 5$ is odd; setting $n=2 k+1$ ( $k$ integer $\geq 2)$, we compute $\frac{n-1}{2}=k$ and $\left[\frac{n+1}{2}\right]+1=k+2$. Hence, from $m-|\beta|-2 h \geq \frac{n-1}{2}$ and $m \leq\left[\frac{n+1}{2}\right]+1$, we find that $k \leq m \leq k+2$. We have to consider three different cases.

ii.1) For $m=k$, inequalities $k \geq m-|\beta|-2 h \geq \frac{n-1}{2}=k$ imply that $|\beta|+2 h=0$ and $|\gamma|+2 l=k$. Then, by Theorem Appendix B.3 b., we get

$$
Z^{\beta} \partial_{1}^{h} u=u \in H_{*, \gamma}^{k}\left(\mathbb{R}_{+}^{n}\right)=H_{*, \gamma}^{\frac{n-1}{2}}\left(\mathbb{R}_{+}^{n}\right) \hookrightarrow L^{r}\left(\mathbb{R}_{+}^{n}\right), \quad \forall r \in[2, n+1[,
$$

and choosing $r=n$ in (B.8) gives

$$
\gamma^{1 / n}\|u\|_{L^{n}\left(\mathbb{R}_{+}^{n}\right)} \leq C\|u\|_{H_{*, \gamma}^{(n-1) / 2}\left(\mathbb{R}_{+}^{n}\right)} .
$$

On the other hand, since $s-k=3$, we have

$$
Z^{\gamma} \partial_{1}^{l} v \in H_{*, \gamma}^{s-k}\left(\mathbb{R}_{+}^{n}\right)=H_{*, \gamma}^{3}\left(\mathbb{R}_{+}^{n}\right) \hookrightarrow L^{2^{*}}\left(\mathbb{R}_{+}^{n}\right) .
$$


We obtain from (B.24), (B.28)

$$
\begin{aligned}
& \gamma^{s-(n+1) / 2}\left\|Z^{\beta} \partial_{1}^{h} u Z^{\gamma} \partial_{1}^{l} v\right\|_{L^{2}\left(\mathbb{R}_{+}^{n}\right)}=\gamma^{2}\left\|u Z^{\gamma} \partial_{1}^{l} v\right\|_{L^{2}\left(\mathbb{R}_{+}^{n}\right)} \\
& \leq \gamma^{1 / n}\|u\|_{L^{n}\left(\mathbb{R}_{+}^{n}\right)} \gamma^{2-1 / n}\left\|Z^{\gamma} \partial_{1}^{l} v\right\|_{L^{2^{*}}\left(\mathbb{R}_{+}^{n}\right)} \leq C\|u\|_{H_{*, \gamma}^{m}\left(\mathbb{R}_{+}^{n}\right)}\|v\|_{H_{*, \gamma}^{s}\left(\mathbb{R}_{+}^{n}\right)} .
\end{aligned}
$$

ii.2) For $m=k+1$, inequality $k+1-|\beta|-2 h \geq \frac{n-1}{2}=k$ gives that $|\beta|+2 h \leq 1$; moreover $k=m-1 \leq|\gamma|+2 l \leq m=k+1$. Since $s-(k+1)=2$, applying again Theorem Appendix B.3 b. for $r=n$ and Corollary Appendix B.4 yields

$$
\begin{aligned}
& Z^{\beta} \partial_{1}^{h} u \in H_{*, \gamma}^{m-1}\left(\mathbb{R}_{+}^{n}\right)=H_{*, \gamma}^{k}\left(\mathbb{R}_{+}^{n}\right)=H_{*, \gamma}^{\frac{n-1}{2}}\left(\mathbb{R}_{+}^{n}\right) \hookrightarrow L^{n}\left(\mathbb{R}_{+}^{n}\right), \\
& Z^{\gamma} \partial_{1}^{l} v \in H_{*, \gamma}^{s-(k+1)}\left(\mathbb{R}_{+}^{n}\right)=H_{*, \gamma}^{2}\left(\mathbb{R}_{+}^{n}\right) \hookrightarrow L^{2^{*}}\left(\mathbb{R}_{+}^{n}\right),
\end{aligned}
$$

and we conclude as in the preceding case.

ii.3) For $m=k+2$, inequality $k+2-|\beta|-2 h \geq k$ implies $|\beta|+2 h \leq 2$. We consider two different cases.

ii.3.1) When $1 \leq|\beta|+2 h \leq 2$ then $k \leq|\gamma|+2 l \leq k+1$. Thus Theorem Appendix B.3 and Corollary Appendix B.4 imply again

$$
\begin{aligned}
& Z^{\beta} \partial_{1}^{h} u \in H_{*, \gamma}^{m-2}\left(\mathbb{R}_{+}^{n}\right)=H_{*, \gamma}^{k}\left(\mathbb{R}_{+}^{n}\right)=H_{*, \gamma}^{\frac{n-1}{2}}\left(\mathbb{R}_{+}^{n}\right) \hookrightarrow L^{n}\left(\mathbb{R}_{+}^{n}\right), \\
& Z^{\gamma} \partial_{1}^{l} v \in H_{*, \gamma}^{s,(k+1)}\left(\mathbb{R}_{+}^{n}\right)=H_{*, \gamma}^{2}\left(\mathbb{R}_{+}^{n}\right) \hookrightarrow L^{2^{*}}\left(\mathbb{R}_{+}^{n}\right),
\end{aligned}
$$

and we conclude as in the preceding case.

ii.3.2) When $|\beta|+2 h=0$, Theorem Appendix B.2 immediately yields

$$
Z^{\beta} \partial_{1}^{h} u=u \in H_{*, \gamma}^{m}\left(\mathbb{R}_{+}^{n}\right)=H_{*, \gamma}^{k+2}\left(\mathbb{R}_{+}^{n}\right)=H_{*, \gamma}^{\left[\frac{n+1}{2}\right]+1}\left(\mathbb{R}_{+}^{n}\right) \hookrightarrow L^{\infty}\left(\mathbb{R}_{+}^{n}\right),
$$

and we conclude as in case i.2.1).

Gathering all the estimates collected in cases i.1), $\cdots$, ii.3.2) above gives the desired estimate for $K_{4}$.

At last, the estimate of $K_{5}$ is deduced by similar arguments; therefore we omit it for shortness.

Gathering all the estimates collected for each of the different terms $K_{1}, \ldots, K_{5}$ before gives that the derivatives $Z^{\alpha} \partial_{1}^{h}(u v) \in L^{2}\left(\mathbb{R}_{+}^{n}\right)$, whenever $|\alpha|+2 k=m$, so that $u v \in H_{*, \gamma}^{m}\left(\mathbb{R}_{+}^{n}\right)$. Combining the found estimates of $K_{1}, \ldots, K_{5}$ with (B.26), (B.27) gives (B.25).

The arguments above require the use of Theorem Appendix B.3, hence the dimension $n$ has to be strictly larger than 3 . We need to treat the cases $n=2$ and $n=3$ separately.

Case $n=2$ : in this case we compute $s=[3 / 2]+2=3$ and what we need to prove is just that $u v \in H_{*, \gamma}^{2}\left(\mathbb{R}_{+}^{2}\right)$, with $\gamma^{3 / 2}\|u v\|_{H_{*, \gamma}^{2}\left(\mathbb{R}_{+}^{2}\right)} \leq C\|u\|_{H_{*, \gamma}^{2}\left(\mathbb{R}_{+}^{2}\right)}\|v\|_{H_{*, \gamma}^{3}\left(\mathbb{R}_{+}^{2}\right)}$, whenever $u \in H_{*, \gamma}^{2}\left(\mathbb{R}_{+}^{2}\right)$ and $v \in H_{*, \gamma}^{3}\left(\mathbb{R}_{+}^{2}\right)$ (recall that the result of Theorem Appendix B.5 is true when $m=1$, for all dimensions $n \geq 2$ ). Note that, for $n=2$, Theorem Appendix B.2 gives the continuous imbedding $H_{*, \gamma}^{2}\left(\mathbb{R}_{+}^{2}\right) \hookrightarrow L^{\infty}\left(\mathbb{R}_{+}^{2}\right)$. In view of $H_{*, \gamma}^{1}\left(\mathbb{R}_{+}^{2}\right) \cdot H_{*, \gamma}^{3}\left(\mathbb{R}_{+}^{2}\right) \hookrightarrow H_{*, \gamma}^{1}\left(\mathbb{R}_{+}^{2}\right)$, we already know that $u v \in H_{*, \gamma}^{1}\left(\mathbb{R}_{+}^{2}\right)$. In order to check that $u v \in H_{*, \gamma}^{2}\left(\mathbb{R}_{+}^{2}\right)$ we still need to show that

$$
\partial_{1}(u v) \in L^{2}\left(\mathbb{R}_{+}^{2}\right) \quad \text { and } \quad Z_{h, j}^{2}(u v) \in L^{2}\left(\mathbb{R}_{+}^{2}\right) .
$$


Leibniz's formula gives $\partial_{1}(u v)=\partial_{1} u v+u \partial_{1} v$; hence $\partial_{1}(u v) \in L^{2}\left(\mathbb{R}_{+}^{2}\right)$, as $\partial_{1} u, \partial_{1} v \in$ $L^{2}\left(\mathbb{R}_{+}^{2}\right)$ and $u, v \in H_{*, \gamma}^{2}\left(\mathbb{R}_{+}^{2}\right) \hookrightarrow L^{\infty}\left(\mathbb{R}_{+}^{2}\right)$. As for the second tangential derivatives, we have

$$
Z_{h}\left(Z_{j}(u v)\right)=Z_{h, j}^{2} u v+Z_{j} u Z_{h} v+Z_{h} u Z_{j} v+u Z_{h, j}^{2} v \in L^{2}\left(\mathbb{R}_{+}^{2}\right),
$$

since again all of the different terms, involved in the right-hand side of the identity above, are products of a function in $L^{2}\left(\mathbb{R}_{+}^{2}\right)$ and a function in $L^{\infty}\left(\mathbb{R}_{+}^{2}\right)$ (because of the continuous imbedding $\left.H_{*, \gamma}^{2}\left(\mathbb{R}_{+}^{2}\right) \hookrightarrow L^{\infty}\left(\mathbb{R}_{+}^{2}\right)\right)$. This proves that $Z_{h, j}^{2} v \in L^{2}\left(\mathbb{R}_{+}^{2}\right)$ and completes the proof that $u v \in H_{*, \gamma}^{2}\left(\mathbb{R}_{+}^{2}\right)$. The a priori estimate in (B.25) is an immediate consequence of (B.1).

Case $n=3$ : The proof for the case $n=3$ follows by similar arguments, by using the continuous imbedding $H_{*, \gamma}^{3}\left(\mathbb{R}_{+}^{3}\right) \hookrightarrow L^{\infty}\left(\mathbb{R}_{+}^{3}\right)$ with (B.1) and usual imbeddings for the standard Sobolev spaces $H_{\gamma}^{m}\left(\mathbb{R}_{+}^{3}\right)$. We omit it for shortness.

This completes the proof of Theorem Appendix B.5 when $1 \leq m<s=\left[\frac{n+1}{2}\right]+2$. If $m=s \geq\left[\frac{n+1}{2}\right]+2$ the result easily follows by induction.

Let us consider the space

$$
V_{\text {tan, } \gamma}^{2}\left(Q_{T}\right)=\left\{F \in H_{\text {tan, },}^{2}\left(Q_{T}\right): F_{\mid t=0} \in H_{\gamma}^{1}\left(\mathbb{R}_{+}^{n}\right)\right\},
$$

equipped with its natural norm. This space has been introduced for Theorem 5.1 in Section 5. We have the following result.

Theorem Appendix B.6. For all integers $m \geq 1$ and for every $\gamma \geq 1, C_{(0)}^{\infty}\left(Q_{T}\right)$ is dense in $V_{\text {tan, },}^{2}\left(Q_{T}\right)$.

Proof. The proof is similar to the proof of Lemma B.3 in [20].

We also define the following space

$$
K_{*, \gamma}^{m}\left(\mathbb{R}_{+}^{n}\right)=H_{t a n, \gamma}^{m}\left(\mathbb{R}_{+}^{n}\right) \cap H_{*, \gamma}^{m-1}\left(\mathbb{R}_{+}^{n}\right), \quad m \geq 1,
$$

equipped with its natural norm

$$
\|u\|_{K_{*, \gamma}^{m}}^{2}=\|u\|_{H_{t a n, \gamma}^{m}}^{2}+\gamma^{2}\|u\|_{H_{*, \gamma}^{m-1}}^{2} .
$$

We observe that $K_{*, \gamma}^{1}=H_{*, \gamma}^{1}=H_{t a n, \gamma}^{1}$. Obviously we also have the continuous imbeddings $K_{*, \gamma}^{m}\left(\mathbb{R}_{+}^{n}\right) \hookrightarrow H_{\text {tan }, \gamma}^{m}\left(\mathbb{R}_{+}^{n}\right)$ and $K_{*, \gamma}^{m}\left(\mathbb{R}_{+}^{n}\right) \hookrightarrow H_{*, \gamma}^{m-1}\left(\mathbb{R}_{+}^{n}\right)$.

Lemma Appendix B.7. Let $n \geq 2$ and $\gamma \geq 1$. For every integer $m \geq\left[\frac{n+1}{2}\right]+2$ the continuous imbedding $K_{*, \gamma}^{m}\left(\mathbb{R}_{+}^{n}\right) \hookrightarrow C_{B}^{0}\left(\mathbb{R}_{+}^{n}\right)$ holds.

Proof. We have $K_{*, \gamma}^{m}\left(\mathbb{R}_{+}^{n}\right) \hookrightarrow H_{*, \gamma}^{m-1}\left(\mathbb{R}_{+}^{n}\right) \hookrightarrow C_{B}^{0}\left(\mathbb{R}_{+}^{n}\right)$, by Theorem Appendix B.2.

The next theorem shows the tangential regularity of the product of two functions, as needed in Section 6.1, with uniform control for $\gamma \geq 1$. 
Theorem Appendix B.8. Let $m \geq 1$ be an integer and $s=\max \left\{m,\left[\frac{n+1}{2}\right]+4\right\}$. If $u \in K_{*, \gamma}^{m}\left(\mathbb{R}_{+}^{n}\right)$ and $v \in H_{*, \gamma}^{s}\left(\mathbb{R}_{+}^{n}\right)$ then $u v \in H_{t a n, \gamma}^{m}\left(\mathbb{R}_{+}^{n}\right)$ and there exists a constant $C$ such that

$$
\gamma^{s-(n+1) / 2}\|u v\|_{H_{t a n, \gamma}^{m}\left(\mathbb{R}_{+}^{n}\right)} \leq C\|u\|_{K_{*, \gamma}^{m}\left(\mathbb{R}_{+}^{n}\right)}\|v\|_{H_{*, \gamma}^{s}\left(\mathbb{R}_{+}^{n}\right)}, \quad \forall \gamma \geq 1 .
$$

If $m=2$ the same result holds with $s=\left[\frac{n+1}{2}\right]+3$ and $\|u\|_{H_{\text {tan, }}^{2}\left(\mathbb{R}_{+}^{n}\right)}$ instead of $\|u\|_{K_{*, \gamma}^{2}\left(\mathbb{R}_{+}^{n}\right)}$.

Proof. i) Consider first the case $1 \leq m \leq 3$. Applying Theorem Appendix B.2 easily gives

$$
\begin{aligned}
& \gamma^{s-(n+1) / 2}\|u v\|_{H_{t a n, \gamma}^{m}\left(\mathbb{R}_{+}^{n}\right)} \leq C \gamma^{s-(n+1) / 2} \sum_{|\alpha| \leq m} \gamma^{m-|\alpha|}\left\|Z^{\alpha}(u v)\right\|_{L^{2}\left(\mathbb{R}_{+}^{n}\right)} \\
& \leq C \sum_{|\alpha| \leq m, \beta \leq \alpha} \gamma^{m-|\alpha-\beta|}\left\|Z^{\alpha-\beta} u\right\|_{L^{2}\left(\mathbb{R}_{+}^{n}\right)} \gamma^{s-|\beta|-(n+1) / 2}\left\|Z^{\beta} v\right\|_{L^{\infty}\left(\mathbb{R}_{+}^{n}\right)} \\
& \leq C\|u\|_{H_{t a n, \gamma}^{m}\left(\mathbb{R}_{+}^{n}\right)} \sum_{|\beta| \leq m}\left\|Z^{\beta} v\right\|_{H_{*, \gamma}^{s-|\beta|}\left(\mathbb{R}_{+}^{n}\right)} \leq C\|u\|_{K_{*, \gamma}^{m}\left(\mathbb{R}_{+}^{n}\right)}\|v\|_{H_{*, \gamma}^{s}\left(\mathbb{R}_{+}^{n}\right)} .
\end{aligned}
$$

If $m=2$, we notice that $s=[(n+1) / 2]+3$ is enough for the estimate on $v$.

ii) Assume now that $m \geq 4$. If (B.30) has already been proved up to $m-1$, then one has

$$
\begin{aligned}
& \gamma^{s-(n+1) / 2}\|u v\|_{H_{\text {tan, }}^{m}\left(\mathbb{R}_{+}^{n}\right)} \\
& \leq C \gamma^{s-(n+1) / 2}\left(\gamma\|u v\|_{H_{\text {tan, }}^{m-1}\left(\mathbb{R}_{+}^{n}\right)}+\|Z u v\|_{H_{t a n, \gamma}^{m-1}\left(\mathbb{R}_{+}^{n}\right)}+\sum_{|\beta|=m}\left\|u Z^{\beta} v\right\|_{L^{2}\left(\mathbb{R}_{+}^{n}\right)}\right) \\
& \leq C\left(\|u\|_{K_{*, \gamma}^{m}\left(\mathbb{R}_{+}^{n}\right)}\|v\|_{H_{*, \gamma}^{s}\left(\mathbb{R}_{+}^{n}\right)}+\gamma^{s-(n+1) / 2} \sum_{|\beta|=m}\left\|u Z^{\beta} v\right\|_{L^{2}\left(\mathbb{R}_{+}^{n}\right)}\right) .
\end{aligned}
$$

Therefore it is enough to prove

$$
\gamma^{s-(n+1) / 2} \sum_{|\beta|=m}\left\|u Z^{\beta} v\right\|_{L^{2}\left(\mathbb{R}_{+}^{n}\right)} \leq C\|u\|_{K_{*, \gamma}^{m}\left(\mathbb{R}_{+}^{n}\right)}\|v\|_{H_{*, \gamma}^{s}\left(\mathbb{R}_{+}^{n}\right)} .
$$

ii.1) If $m=4$ and $n=2$ (this yields $s=5$ ), then $u \in K_{*, \gamma}^{4}\left(\mathbb{R}_{+}^{2}\right) \hookrightarrow H_{*, \gamma}^{2}\left(\mathbb{R}_{+}^{2}\right) \hookrightarrow$ $L^{\infty}\left(\mathbb{R}_{+}^{2}\right)$ (by Theorem Appendix B.2) and the estimate (B.30) follows from

$$
\begin{aligned}
& \gamma^{5-3 / 2} \sum_{|\beta|=4}\left\|u Z^{\beta} v\right\|_{L^{2}\left(\mathbb{R}_{+}^{2}\right)} \leq \gamma^{5-3 / 2}\|u\|_{L^{\infty}\left(\mathbb{R}_{+}^{2}\right)} \sum_{|\beta|=4}\left\|Z^{\beta} v\right\|_{L^{2}\left(\mathbb{R}_{+}^{2}\right)} \\
& \leq C \gamma^{3}\|u\|_{H_{*, \gamma}^{2}\left(\mathbb{R}_{+}^{2}\right)}\|v\|_{H_{\text {tan, }}^{4}\left(\mathbb{R}_{+}^{2}\right)} \leq C\|u\|_{K_{*, \gamma}^{4}\left(\mathbb{R}_{+}^{2}\right)}\|v\|_{H_{*, \gamma}^{5}\left(\mathbb{R}_{+}^{2}\right) .}
\end{aligned}
$$

ii.2) If $m=4$ and $n=3$, a similar calculation gives the same result (with $s=6$ ).

ii.3) If $m=4$ and $n \geq 4$, then $u \in H_{*, \gamma}^{2}\left(\mathbb{R}_{+}^{n}\right) \hookrightarrow L^{2^{*}}\left(\mathbb{R}_{+}^{n}\right)$. If $n \geq 4$ is even, for $|\beta|=4, Z^{\beta} v \in H_{*, \gamma}^{[(n+1) / 2]}\left(\mathbb{R}_{+}^{n}\right)=H_{*, \gamma}^{n / 2}\left(\mathbb{R}_{+}^{n}\right) \hookrightarrow L^{n}\left(\mathbb{R}_{+}^{n}\right)$, by (B.9). We may apply (B.11), (B.24) and we get

$$
\begin{aligned}
& \gamma^{s-(n+1) / 2} \sum_{|\beta|=4}\left\|u Z^{\beta} v\right\|_{L^{2}\left(\mathbb{R}_{+}^{n}\right)} \leq \gamma^{1-1 / n}\|u\|_{L^{2^{*}\left(\mathbb{R}_{+}^{n}\right)}} \gamma^{1 / 2+1 / n} \sum_{|\beta|=4}\left\|Z^{\beta} v\right\|_{L^{n}\left(\mathbb{R}_{+}^{n}\right)} \\
& \leq C\|u\|_{H_{*, \gamma}^{2}\left(\mathbb{R}_{+}^{n}\right)} \sum_{|\beta|=4}\left\|Z^{\beta} v\right\|_{H_{*, \gamma}^{n / 2}\left(\mathbb{R}_{+}^{n}\right)} \leq C\|u\|_{K_{*, \gamma}^{4}\left(\mathbb{R}_{+}^{n}\right)}\|v\|_{H_{*, \gamma}^{s}\left(\mathbb{R}_{+}^{n}\right)} .
\end{aligned}
$$


If $n \geq 5$ is odd, the thesis follows again because $Z^{\beta} v \in H_{*, \gamma}^{[(n+1) / 2]}\left(\mathbb{R}_{+}^{n}\right) \hookrightarrow$ $H_{*, \gamma}^{(n-1) / 2}\left(\mathbb{R}_{+}^{n}\right) \hookrightarrow L^{n}\left(\mathbb{R}_{+}^{n}\right)$, by (B.7). Applying (B.8) with $r=n$ and (B.24) yields

$$
\begin{aligned}
& \gamma^{s-(n+1) / 2} \sum_{|\beta|=4}\left\|u Z^{\beta} v\right\|_{L^{2}\left(\mathbb{R}_{+}^{n}\right)} \\
& \leq \gamma^{s-(n+1) / 2-1} \gamma^{1-1 / n}\|u\|_{L^{2^{*}}\left(\mathbb{R}_{+}^{n}\right)} \sum_{|\beta|=4} \gamma^{1 / n}\left\|Z^{\beta} v\right\|_{L^{n}\left(\mathbb{R}_{+}^{n}\right)} \\
& \leq C \gamma^{s-(n+1) / 2-1}\|u\|_{H_{*, \gamma}^{2}\left(\mathbb{R}_{+}^{n}\right)} \sum_{|\beta|=4}\left\|Z^{\beta} v\right\|_{H_{*, \gamma}^{(n-1) / 2}\left(\mathbb{R}_{+}^{n}\right)} \leq C\|u\|_{K_{*, \gamma}^{4}\left(\mathbb{R}_{+}^{n}\right)}\|v\|_{H_{*, \gamma}^{s}\left(\mathbb{R}_{+}^{n}\right)} .
\end{aligned}
$$

iii) Let us assume $m=5$. If $2 \leq n \leq 6$, then the estimate (B.31) follows by a calculation similar to that for (B.32), because $u \in K_{*, \gamma}^{5}\left(\mathbb{R}_{+}^{n}\right) \hookrightarrow H_{*, \gamma}^{4}\left(\mathbb{R}_{+}^{n}\right) \hookrightarrow$ $L^{\infty}\left(\mathbb{R}_{+}^{n}\right)$ (by Theorem Appendix B.2). For $n \geq 7$ we need to distinguish again if $n$ is either even or odd. We begin with the case $n$ even.

iii.1) If $n=8$, then $u \in H_{*, \gamma}^{4}\left(\mathbb{R}_{+}^{8}\right) \hookrightarrow L^{16}\left(\mathbb{R}_{+}^{8}\right)$ and

$$
\gamma^{1 / 16}\|u\|_{L^{16}\left(\mathbb{R}_{+}^{8}\right)} \leq C\|u\|_{H_{*, \gamma}^{4}\left(\mathbb{R}_{+}^{8}\right)},
$$

by (B.9), (B.10). On the other hand, for $|\beta|=5, Z^{\beta} v \in H_{*, \gamma}^{3}\left(\mathbb{R}_{+}^{8}\right) \hookrightarrow L^{16 / 7}\left(\mathbb{R}_{+}^{8}\right)$, and

$$
\gamma^{3-9 / 16}\left\|Z^{\beta} v\right\|_{L^{16 / 7}\left(\mathbb{R}_{+}^{8}\right)} \leq C\left\|Z^{\beta} v\right\|_{H_{*, \gamma}^{3}\left(\mathbb{R}_{+}^{8}\right)},
$$

by (B.5), (B.6). Therefore

$$
\begin{aligned}
& \gamma^{8-9 / 2} \sum_{|| \beta \mid=5}\left\|u Z^{\beta} v\right\|_{L^{2}\left(\mathbb{R}_{+}^{8}\right)} \\
& \leq \gamma^{8-9 / 2-(3-1 / 2)} \gamma^{1 / 16}\|u\|_{L^{16}\left(\mathbb{R}_{+}^{8}\right)} \sum_{|\beta|=5} \gamma^{3-9 / 16}\left\|Z^{\beta} v\right\|_{L^{16 / 7}\left(\mathbb{R}_{+}^{8}\right)} \\
& \leq C \gamma\|u\|_{H_{*, \gamma}^{4}\left(\mathbb{R}_{+}^{8}\right)} \sum_{|\beta|=5}\left\|Z^{\beta} v\right\|_{H_{*, \gamma}^{3}\left(\mathbb{R}_{+}^{8}\right)} \leq C\|u\|_{K_{*, \gamma}^{5}\left(\mathbb{R}_{+}^{8}\right)}\|v\|_{H_{*, \gamma}^{8}\left(\mathbb{R}_{+}^{8}\right)} .
\end{aligned}
$$

iii.2) If $n \geq 10$ is even, then $u \in H_{*, \gamma}^{4}\left(\mathbb{R}_{+}^{n}\right) \hookrightarrow L^{r^{*}}\left(\mathbb{R}_{+}^{n}\right)$ and

$$
\|u\|_{L^{r^{*}\left(\mathbb{R}_{+}^{n}\right)}} \leq C\|u\|_{H_{*, \gamma}^{4}\left(\mathbb{R}_{+}^{n}\right)},
$$

by (B.5), (B.6), where $1 / r^{*}=1 / 2-4 /(n+1)$. On the other hand, for $|\beta|=5$, $Z^{\beta} v \in H_{*, \gamma}^{s-5}\left(\mathbb{R}_{+}^{n}\right) \hookrightarrow L^{(n+1) / 4}\left(\mathbb{R}_{+}^{n}\right)$, and

$$
\gamma^{5 / 2}\left\|Z^{\beta} v\right\|_{L^{(n+1) / 4}\left(\mathbb{R}_{+}^{n}\right)} \leq C\left\|Z^{\beta} v\right\|_{H_{*, \gamma}^{s-5}\left(\mathbb{R}_{+}^{n}\right)},
$$

again by (B.5), (B.6). Therefore

$$
\begin{aligned}
& \gamma^{s-(n+1) / 2} \sum_{|\beta|=5}\left\|u Z^{\beta} v\right\|_{L^{2}\left(\mathbb{R}_{+}^{n}\right)} \\
& \leq \gamma^{s-(n+1) / 2-5 / 2}\|u\|_{L^{*}\left(\mathbb{R}_{+}^{n}\right)} \sum_{|\beta|=5} \gamma^{5 / 2}\left\|Z^{\beta} v\right\|_{L^{(n+1) / 4}\left(\mathbb{R}_{+}^{n}\right)} \\
& \leq C \gamma\|u\|_{H_{*, \gamma}^{4}\left(\mathbb{R}_{+}^{n}\right)} \sum_{|\beta|=5}\left\|Z^{\beta} v\right\|_{H_{*, \gamma}^{s-5}\left(\mathbb{R}_{+}^{n}\right)} \leq C\|u\|_{K_{*, \gamma}^{5}\left(\mathbb{R}_{+}^{n}\right)}\|v\|_{H_{*, \gamma}^{s}\left(\mathbb{R}_{+}^{n}\right)} .
\end{aligned}
$$


Now we assume that $n \geq 7$ is odd. In such a case (B.31) follows again by the Hölder inequality and suitable estimates, detailed as follows.

iii.3) If $n=7$, then $u \in H_{*, \gamma}^{3}\left(\mathbb{R}_{+}^{7}\right) \hookrightarrow L^{14 / 3}\left(\mathbb{R}_{+}^{7}\right)$ and

$$
\gamma^{5 / 7}\|u\|_{L^{14 / 3}\left(\mathbb{R}_{+}^{7}\right)} \leq C\|u\|_{H_{*, \gamma}^{3}\left(\mathbb{R}_{+}^{7}\right)},
$$

by (B.7), (B.8). On the other hand, for $|\beta|=5, Z^{\beta} v \in H_{*, \gamma}^{3}\left(\mathbb{R}_{+}^{7}\right) \hookrightarrow L^{7 / 2}\left(\mathbb{R}_{+}^{7}\right)$, and

$$
\gamma^{9 / 7}\left\|Z^{\beta} v\right\|_{L^{7 / 2}\left(\mathbb{R}_{+}^{7}\right)} \leq C\left\|Z^{\beta} v\right\|_{H_{*, \gamma}^{3}\left(\mathbb{R}_{+}^{7}\right)},
$$

again by (B.7), (B.8).

iii.4) If $n=9$ the proof is similar. We have $u \in H_{*, \gamma}^{4}\left(\mathbb{R}_{+}^{9}\right) \hookrightarrow L^{18 / 5}\left(\mathbb{R}_{+}^{9}\right)$ and

$$
\gamma^{16 / 9}\|u\|_{L^{18 / 5\left(\mathbb{R}_{+}^{9}\right)}} \leq C\|u\|_{H_{*, \gamma}^{4}\left(\mathbb{R}_{+}^{9}\right)},
$$

by (B.7), (B.8). On the other hand, for $|\beta|=5, Z^{\beta} v \in H_{*, \gamma}^{4}\left(\mathbb{R}_{+}^{9}\right) \hookrightarrow L^{9 / 2}\left(\mathbb{R}_{+}^{9}\right)$, and

$$
\gamma^{11 / 9}\left\|Z^{\beta} v\right\|_{L^{9 / 2}\left(\mathbb{R}_{+}^{9}\right)} \leq C\left\|Z^{\beta} v\right\|_{H_{*, \gamma}^{4}\left(\mathbb{R}_{+}^{9}\right)},
$$

again by (B.7), (B.8).

iii.5) If $n \geq 11$ is odd, then $u \in H_{*, \gamma}^{4}\left(\mathbb{R}_{+}^{n}\right) \hookrightarrow L^{r^{*}}\left(\mathbb{R}_{+}^{n}\right)$ and

$$
\|u\|_{L^{r^{*}\left(\mathbb{R}_{+}^{n}\right)}} \leq C\|u\|_{H_{*, \gamma}^{4}\left(\mathbb{R}_{+}^{n}\right)},
$$

by (B.5), (B.6), where $1 / r^{*}=1 / 2-4 /(n+1)$. On the other hand, for $|\beta|=5$, $Z^{\beta} v \in H_{*, \gamma}^{s-5}\left(\mathbb{R}_{+}^{n}\right) \hookrightarrow L^{(n+1) / 4}\left(\mathbb{R}_{+}^{n}\right)$, and

$$
\gamma^{3}\left\|Z^{\beta} v\right\|_{L^{(n+1) / 4}\left(\mathbb{R}_{+}^{n}\right)} \leq C\left\|Z^{\beta} v\right\|_{H_{*, \gamma}^{s-5}\left(\mathbb{R}_{+}^{n}\right)},
$$

by (B.7), (B.8).

iv) Then we consider the case $6 \leq m \leq\left[\frac{n+1}{2}\right]+1$ (which yields $n \geq 9$ ), and $s=\left[\frac{n+1}{2}\right]+4$, where we proceed by finite induction. We assume that (B.30) holds for $m-1$ and prove it for $m$. Again, it is enough to show (B.31). For $|\beta|=m$, $Z^{\beta} v \in H_{*, \gamma}^{s-m}\left(\mathbb{R}_{+}^{n}\right)$ with $3 \leq s-m<\frac{n-1}{2}$. Then by (B.5), (B.6), $Z^{\beta} v \in L^{r^{*}}\left(\mathbb{R}_{+}^{n}\right)$, where $\frac{1}{r^{*}}=\frac{1}{2}-\frac{s-m}{n+1}$, and

$$
\left\|Z^{\beta} v\right\|_{L^{r^{*}\left(\mathbb{R}_{+}^{n}\right)}} \leq C\left\|Z^{\beta} v\right\|_{H_{*, \gamma}^{s-m}\left(\mathbb{R}_{+}^{n}\right)} .
$$

On the other hand, $u \in H_{*, \gamma}^{m-1}\left(\mathbb{R}_{+}^{n}\right)$. Here we have three cases.

iv.1) If $m-1<\frac{n-1}{2}$, by (B.5), (B.6), $u \in L^{p}\left(\mathbb{R}_{+}^{n}\right)$, where $\frac{1}{p}=\frac{s-m}{n+1}$ and

$$
\gamma^{s-1-(n+1) / 2}\|u\|_{L^{p}\left(\mathbb{R}_{+}^{n}\right)} \leq C\|u\|_{H_{*, \gamma}^{m-1}\left(\mathbb{R}_{+}^{n}\right)} .
$$

Therefore the thesis follows from (B.35), (B.36).

iv.2) If $m-1=\frac{n-1}{2}$ then $n$ is odd. We get again (B.36) (with the same $p$ ) from (B.8). The thesis follows as before.

iv.3) If $m-1>\frac{n-1}{2}$ then $m=\left[\frac{n+1}{2}\right]+1$. If $n$ is odd we apply the same argument as in iv.2). If $n$ is even then $m-1=n / 2$ and we use (B.9), (B.10) in order to get (B.36) (with the same $p$ ). The thesis follows as before. 
v) Finally we consider $m \geq\left[\frac{n+1}{2}\right]+2$. In this case the thesis easily follows from (B.31), because $u \in H_{*, \gamma}^{m-1}\left(\mathbb{R}_{+}^{n}\right) \hookrightarrow L^{\infty}\left(\mathbb{R}_{+}^{n}\right)$ with

$$
\gamma^{m-1-(n+1) / 2}\|u\|_{L^{\infty}\left(\mathbb{R}_{+}^{n}\right)} \leq C\|u\|_{H_{*, \gamma}^{m-1}\left(\mathbb{R}_{+}^{n}\right)},
$$

as follows from Theorem Appendix B.2. The proof is complete.

Let's give the regularity of the matrices $\Gamma_{\beta}, \Gamma_{0}, \Psi$ in (5.6).

Lemma Appendix B.9. Let $\sigma$ be an integer such that $\sigma \geq[(n+1) / 2]+4$. Assume that $A_{j} \in \mathcal{C}_{T}\left(H_{*, \gamma}^{\sigma}\right)$, for $j=1, \ldots, n, B \in \mathcal{C}_{T}\left(H_{*, \gamma}^{\sigma-2}\right)$. Then the matrices $\Gamma_{\beta}, \Gamma_{0}, \Psi$ of formula (5.6) satisfy

$$
\Gamma_{\beta} \in \mathcal{C}_{T}\left(H_{*, \gamma}^{\sigma-3}\right), \quad \Gamma_{0} \in \mathcal{C}_{T}\left(H_{*, \gamma}^{\sigma-2}\right), \quad \Psi \in \mathcal{C}_{T}\left(H_{*, \gamma}^{\sigma-1}\right) .
$$

Under the same assumption for $A_{j}$, if $B \in \mathcal{C}_{T}\left(H_{*, \gamma}^{\sigma-1}\right)$ then $\Gamma_{0} \in \mathcal{C}_{T}\left(H_{*, \gamma}^{\sigma-1}\right)$.

Proof. See [18], Lemma C.1.

Finally, we give some lemmata useful in the proof of the main Theorem 1.2.

Lemma Appendix B.10. Let $\sigma \geq[(n+1) / 2]+3$ and let $A$ be a matrix-valued function such that $A \in H_{*, \gamma}^{\sigma}\left(\mathbb{R}_{+}^{n}\right)$ and $A=0$ if $x_{1}=0$. Then, for each regular enough vector-valued function $u$

$$
\left\|A \partial_{1} u\right\|_{L^{2}\left(\mathbb{R}_{+}^{n}\right)} \leq c\|A\|_{H_{*, \gamma}^{\sigma}\left(\mathbb{R}_{+}^{n}\right)}\left\|Z_{1} u\right\|_{L^{2}\left(\mathbb{R}_{+}^{n}\right)} .
$$

Proof. See [18], Lemma B.9.

Lemma Appendix B.11. Let $\sigma \geq 2$. Let $A \in H_{*, \gamma}^{\sigma}\left(\mathbb{R}_{+}^{n}\right)$ be a matrix-valued function such that $A=0$ if $x_{1}=0$ and let $H$ be defined as in the proof of Lemma Appendix B.10. Then

$$
\|H\|_{H_{*, \gamma}^{\sigma-2}\left(\mathbb{R}_{+}^{n}\right)} \leq c\|A\|_{H_{*, \gamma}^{\sigma}\left(\mathbb{R}_{+}^{n}\right)}
$$

\section{References}

[1] M. S. Agranovič, Boundary value problems for systems with a parameter, Mat. Sb. (N.S.) 84(126) (1971) 27-65.

[2] H. Beirão da Veiga, Perturbation theory and well-posedness in Hadamard's sense of hyperbolic initial-boundary value problems, Nonlinear Anal., Theory Methods Appl. 22(10) (1994) 1285-1308.

[3] S. Benzoni-Gavage, F. Rousset, D. Serre and K. Zumbrun, Generic types and transitions in hyperbolic initial-boundary-value problems, Proc. Roy. Soc. Edinburgh Sect. A 132 (2002) 1073-1104.

[4] S. Benzoni-Gavage and D. Serre, Multidimensional hyperbolic partial differential equations, Oxford Mathematical Monographs. The Clarendon Press Oxford University Press, Oxford, 2007. First-order systems and applications.

[5] J. Bergh and J. Löfström. Interpolation spaces. An introduction. Springer-Verlag, Berlin, 1976. Grundlehren der Mathematischen Wissenschaften, No. 223. 
[6] E. Casella, P. Secchi and P. Trebeschi, Non-homogeneous linear symmetric hyperbolic systems with characteristic boundary, Differential Integral Equations 19(1) (2006) $51-74$.

[7] J. Chazarain and A. Piriou. Introduction to the theory of linear partial differential equations, volume 14 of Studies in Mathematics and its Applications. North-Holland Publishing Co., Amsterdam, 1982. Translated from the French.

[8] J. F. Coulombel. Stabilité multidimensionnelle d'interfaces dynamiques. applications aux transitions de phase liquide-vapeur. PhD thesis, 2002.

[9] J. F. Coulombel, Well-posedness of hyperbolic initial boundary value problems, $J$. Math. Pure Appl. 84 (2005) 786-818.

[10] J. F. Coulombel and O. Guès, Geometric optics expansions with amplification for hyperbolic boundary value problems: linear problems, Ann. Inst. Fourier (Grenoble), to appear.

[11] J. F. Coulombel and P. Secchi, The stability of compressible vortex sheets in two space dimensions, Indiana Univ. Math. J. 53(4) (2004) 941-1012.

[12] J. F. Coulombel and P. Secchi, Nonlinear compressible vortex sheets in two space dimensions, Ann. Sci. École Norm. Sup. (4) 41(1) (2008) 85-139.

[13] J. F. Coulombel and P. Secchi, Uniqueness of 2-D compressible vortex sheets, Commun. Pure Appl. Anal. 8(4) (2009) 1439-1450.

[14] L. Hörmander, Linear partial differential operators, Springer Verlag, Berlin, 1976.

[15] A. Majda, The stability of multidimensional shock fronts, Mem. Amer. Math. Soc., 41(275):iv+95, 1983.

[16] A. Majda, Compressible fluid flow and systems of conservation laws in several space variables, Springer-Verlag, 1984.

[17] A. Morando and P. Secchi, Regularity of weakly well posed characteristic boundary value problems, Quad. Sem. Mat. Univ. Brescia n. 6, 2010.

[18] A. Morando, P. Secchi and P. Trebeschi, Regularity of solutions to characteristic initial-boundary value problems for symmetrizable systems, J. Hyperbolic Differ. Equ. 6(4) (2009) 753-808.

[19] T. Nishitani and M. Takayama, Regularity of solutions to non-uniformly characteristic boundary value problems for symmetric systems, Comm. Partial Differential Equations 25(5-6) (2000) 987-1018.

[20] M. Ohno, Y. Shizuta and T. Yanagisawa, The initial-boundary value problem for linear symmetric hyperbolic systems with boundary characteristic of constant multiplicity, J. Math. Kyoto Univ. 35(2) (1995) 143-210.

[21] J. Rauch, Symmetric positive systems with boundary characteristic of constant multiplicity, Trans. Amer. Math. Soc. 291(1) (1985) 167-187.

[22] J. Rauch and F. J. Massey III, Differentiability of solutions to hyperbolic initialboundary value problems, Trans. Amer. Math. Soc. 189 (1974) 303-318.

[23] M. Sablé-Tougeron, Existence pour un problème de l'élastodynamique Neumann non linéaire en dimension 2, Arch. Rational Mech. Anal. 101(3) (1988) 261-292.

[24] P. Secchi, Linear symmetric hyperbolic systems with characteristic boundary, Math. Methods Appl. Sci. 18(11) (1995) 855-870.

[25] P. Secchi, The initial-boundary value problem for linear symmetric hyperbolic systems with characteristic boundary of constant multiplicity, Differential Integral Equations 9(4) (1996) 671-700.

[26] P. Secchi, Characteristic symmetric hyperbolic systems with dissipation: Global existence and asymptotics, Math. Methods Appl. Sci. 20(7) (1997) 583-597.

[27] P. Secchi, Some properties of anisotropic Sobolev spaces, Arch. Math. (Basel) 75(3) (2000) 207-216. 
58 A. Morando \& P. Secchi

[28] M. E. Taylor, Rayleigh waves in linear elasticity as a propagation of singularities phenomenon, In Partial differential equations and geometry (Proc. Conf., Park City, Utah, 1977), volume 48 of Lecture Notes in Pure and Appl. Math., pages 273-291. Dekker, New York, 1979. 